\title{
The Dynamics of Exploitation and Class in Accumulation Economies*
}

\author{
Jonathan F. Cogliano, ${ }^{\dagger}$ Roberto Veneziani, ${ }^{\ddagger}$ and Naoki Yoshihara ${ }^{\S}$
}

December 10, 2014

\begin{abstract}
This paper analyses the equilibrium dynamics of exploitation and class in general accumulation economies with population growth, technical change, and bargaining by adopting a novel computational approach. First, the determinants of the emergence and persistence of exploitation and class are investigated, and the role of labour-saving technical change and, even more importantly, power is highlighted. Second, it is shown that the concept of exploitation provides the foundations for a logically coherent and empirically relevant analysis of inequalities and class relations in advanced capitalist economies. An index that identifies the exploitation level, or intensity of each individual can be defined and its empirical distribution studied using the standard tools developed in the theory of inequality measurement.

JEL: B51 (Socialist, Marxian, Sraffian); D63 (Equity, Justice, Inequality, and Other Normative Criteria and Measurement); C63 (Computational Techniques, Simulation Modeling).
\end{abstract}

Keywords: Exploitation, class, accumulation, simulation.

*We would like to thank Marco Mariotti for his thoughtful comments and suggestions. The usual disclaimer applies.

${ }^{\dagger}$ (Corresponding author) Department of Economics, Dickinson College, Althouse 112, P.O. Box 1773 Carlisle, PA 17013, U.S. (coglianj@dickinson.edu)

${ }^{\ddagger}$ School of Economics and Finance, Queen Mary University of London, Mile End Road, London E1 4NS, UK. (r.veneziani@qmul.ac.uk)

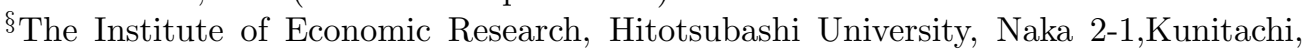
Tokyo 186-0004, Japan. (yosihara@ier.hit-u.ac.jp) 


\section{Introduction}

One of the key tenets of Marxian economics is the idea that exploitation and class are defining features of capitalist economies. This raises two issues. First, the existence of a logically coherent and empirically meaningful definition of exploitation and class. Second, the economic mechanisms that lead to the emergence and persistence of exploitation and class.

The first issue has received a lot of attention in the literature, leading to vast debates on the notions of exploitation and class. The received view is that no logically consistent and empirically meaningful definition exists which captures the key positive and normative insights of the Marxian theory of exploitation and class in general economies. This view has been questioned in a series of recent papers by Yoshihara and Veneziani (e.g. [26, 27, 19]). In these contributions, the concept of exploitation is analysed using a novel, general axiomatic approach which allows one to rigorously capture the normative and positive foundations of exploitation theory. Contrary to the received wisdom, it is shown that there exists a nonempty class of logically consistent definitions - conceptually related to the so-called "New Interpretation" (Duménil [5]; Foley [8]; Duménil, Foley, and Lévy [6]; see also Mohun [11]) - that satisfy a set of desirable properties in general convex economies with heterogeneous agents - including the existence of a robust relation between profits and exploitation, as well as between class and exploitation.

These contributions, however, focus on one-period economies with no savings and accumulation, and have relatively little to say about the second issue in the opening paragraph, namely the dynamics of class, exploitation, and profits. This is not a minor issue. In his seminal theory, Roemer [14] has proved that exploitation and classes emerge as the equilibrium outcome of differential ownership of the means of production in competitive economies with optimising agents when capital is scarce, leading him to conclude that the normative relevance of exploitation reduces to an exclusive emphasis on asset inequalities.

Yet Roemer's results have been derived in one-period models whereas capitalism, according to Marx, is an inherently dynamic system geared towards capital accumulation and so one may argue that class and exploitation should be analysed in a dynamic framework. In dynamic accumulation economies, it is not difficult to show that capital may become abundant, leading profits and exploitation to disappear (Devine and Dymski [4]). Perhaps more surprisingly, Veneziani [17, 18] and Veneziani and Yoshihara [20] have proved that if savings are allowed in a dynamic capitalist economy, then asset in-

equalities are necessary for exploitation to emerge, but alone they are not 
sufficient for it to persist even if agents do not accumulate in equilibrium. These results cast doubts on the claim that asset inequalities are necessary and sufficient for the emergence and the persistence of exploitative relations, and raise the issue of the determinants of exploitation and class.

This paper adopts the conceptual approach to exploitation proposed by Yoshihara and Veneziani $([26,27,19])$ in order to study the dynamics of asset inequalities, exploitation and classes. We significantly generalise Roemer's $[13,14]$ accumulation economies with maximising agents in order to incorporate nonstationary prices, population growth, time-varying consumption norms, technical change, and distributive conflict. We analyse - both formally and computationally - the dynamic equilibrium trajectories of the economies and their class and exploitation structures, and generalise some fundamental insights originally proved by Roemer [14], including the so-called Class-Exploitation Correspondence Principle (henceforth, CECP).

To be specific, we consider three main models exploring different mechanisms determining the emergence and persistence of exploitation and class. We start off by analysing a basic economy with constant consumption, population, and technology: this benchmark scenario confirms the insights of the previous literature by showing that accumulation leads exploitation to disappear because the economy eventually becomes labour constrained.

We then extend the model to consider capitalists' decisions to innovate and norm-based consumption dynamics. Empirically, the long-run evolution of capitalist economies has indeed been characterised by an increase in (average) consumption opportunities and by a tendential expansion of technical knowledge, leading to a progressive increase in labour productivity (Flaschel et al. [7]). Theoretically, our analysis confirms that labour-saving technical progress may play a crucial role in making exploitation persistent by guaranteeing the persistent abundance of labour (Skillman [15]). In other words, the capitalists' control of investment and innovation decisions can make exploitation persistent by maintaining labour unemployment over time.

Although many actual capitalist economies have indeed gone through long spells of labour unemployment, one may argue with Roemer [14] that a general theory of exploitation and classes should not crucially depend either positively or normatively - on structural imbalances in factor markets. Exploitation and class are characteristics of capitalist relations of production and full employment does not make capitalist economies non-exploitative. Therefore we analyse an extension of the basic model with population growth, technical change and accumulation in which full employment occurs in every period and distribution is determined using a general Nash bargaining procedure with the bargaining power of each agent endogenously determined as 
a function of their ownership of the means of production and class solidarity. The results are quite striking: technical change and population growth are not sufficient to make exploitation persistent unless capitalists are sufficiently powerful and class solidarity among propertyless workers is sufficiently weak even if the economy never becomes labour constrained. Capitalist power is an essential determinant of the persistence of exploitation and class.

In all economies, we analyse the evolution of the structure of exploitative relations. By deriving a robust correspondence between class and exploitation status, the CECP yields relevant normative insights on capitalist economies. Yet, the CECP draws a rather partial, coarse picture of the structure of exploitative relations: two economies with similar numbers of agents belonging to each class and each exploitation category may still be very different. Based on $[26,27,19]$, we propose a novel index of the level, or intensity of exploitation for individual agents, whose distribution provides a finer and more comprehensive picture of exploitative relations. The analysis of its distribution yields relevant normative insights, and it raises some interesting issues that are conceptually analogous to those discussed in the literature on the measurement of income inequality.

Another contribution of the paper is methodological. Given the complexity of the economies considered, the paper adopts a novel computational approach to Marxian exploitation theory. Pioneering work applying computational methods to Marxian theory includes Wright [23, 24, 25], Cogliano [2], and Cogliano and Jiang [3]. But the latter contributions focus on Marxian price and value theory and the circuit of capital rather than exploitation and class. More related to our work is an unpublished paper by Takamasu [16], which adopts a computational approach to study class formation in accumulation economies. Yet this paper does not analyse exploitation and it only considers a very basic scenario with constant technology, population and consumption. Moreover, there is no explicit analysis of agents' maximising decisions or of the equilibrium conditions.

By moving beyond the straightjacket of analytical solutions, a computational approach allows us to study the equilibrium determination of exploitation status and the Class-Exploitation Correspondence Principle, and trace the co-evolution of exploitation and class over time in complex economies with endogenous technical change, population growth, norm-based consumption dynamics, and generalised $N$-agent bargaining. The results obtained are robust with respect to changes in the specification of technology, population, preferences and, especially, endowments, but also to alternative specifications of some of the behavioural assumptions.

The rest of the paper is structured as follows. The general framework 
is described in section 2. Section 3 analyses the benchmark economy with stationary technology, population and consumption. Section 4 derives some key theoretical results concerning class and exploitation in the basic model. Section 5 presents the index capturing the level of exploitation of each agent. Section 6 analyses the dynamics of the basic model, and its exploitation and class structures, using computational techniques. Section 7 extends the analysis to economies with endogenous consumption and technical change, whereas section 8 focuses on the role of bargaining and power in economies with full employment, technical change and population growth. Section 9 discusses the robustness of the results. Section 10 concludes.

\section{The framework}

Consider a dynamic extension of Roemer's [14] accumulating economy with a labour market and only one good produced and consumed. ${ }^{1}$ In every period $t=1,2, \ldots$, let $\mathcal{N}_{t}$ denote the set of agents with cardinality $N_{t}$ and generic element $\nu$. At the beginning of each production period $t$, there is a finite set, $\mathcal{P}_{t}$, of Leontief production techniques $\left(A_{t}, L_{t}\right)$, where $0<A_{t}<1$ and $L_{t}>0$, and all agents have access to the techniques in $\mathcal{P}_{t}$.

In every period $t$, agents have identical preferences but possess potentially different endowments of labour, $l_{t-1}^{\nu}$, and capital, $\omega_{t-1}^{\nu}$, inherited from previous periods. The distribution of endowments at the beginning of $t$ is given by $\Pi_{t-1}=\left(l_{t-1}^{\nu}\right)_{\nu \in \mathcal{N}_{t}} \in \mathbb{R}_{++}^{N}$ and $\Omega_{t-1}=\left(\omega_{t-1}^{\nu}\right)_{\nu \in \mathcal{N}_{t}} \in \mathbb{R}_{+}^{N}$. In every $t$, each agent $\nu \in \mathcal{N}_{t}$ is therefore completely identified by a duplet $\left(l_{t-1}^{\nu}, \omega_{t-1}^{\nu}\right) \in \mathbb{R}_{+}^{2}$. An agent $\nu \in \mathcal{N}_{t}$ endowed with $\left(l_{t-1}^{\nu}, \omega_{t-1}^{\nu}\right)$ can engage in three types of production activity: she can sell her labour power $z_{t}^{\nu}$; she can hire others to operate a technique $\left(A_{t}, L_{t}\right) \in \mathcal{P}_{t}$ at the level $y_{t}^{\nu}$; or she can work on her own to operate $\left(A_{t}, L_{t}\right) \in \mathcal{P}_{t}$ at the level $x_{t}^{\nu}$.

Following Roemer $[13,14]$, we assume that production takes time and current choices are constrained by past events. To be precise, wages are paid ex post and $w_{t} \in \mathbb{R}_{+}$denotes the nominal wage rate at the end of $t$, but every agent must be able to lay out in advance the operating costs for the activities she chooses to operate using her wealth $W_{t-1}^{\nu}$. Letting $p_{t} \in \mathbb{R}_{+}$denote the price of the produced commodity at the end of $t$ and beginning of $t+1$, the market value of agent $\nu$ 's endowment - her wealth - is $W_{t-1}^{\nu}=p_{t-1} \omega_{t-1}^{\nu}$. The

\footnotetext{
${ }^{1}$ Given our focus on the dynamics of exploitation and class, the one-good assumption yields no loss of generality. The model can be extended to include $n$ commodities, albeit at the cost of a significant increase in technicalities and computational intensity.
} 
wealth that is not used for production activities can be invested to purchase goods to sell at the end of the period, $\delta_{t}^{\nu}$.

Our main behavioural assumption postulates that agents wish to accumulate as much as possible, subject to consuming $b_{t} \in \mathbb{R}_{++}$per unit of labour performed, $\Lambda_{t}^{\nu} \equiv L x_{t}^{\nu}+z_{t}^{\nu}$. Within every period $t$, we consider $b_{t}$ as a constant parameter, but we do allow for the possibility that $b_{t}$ changes endogenously over time.

This modelling choice is motivated by our focus on the dynamics of exploitation and class in capitalist economies characterised by a drive to accumulate, rather than on consumer choices. Theoretically, it is also consistent with the classical-Marxian tradition where consumption is largely the product of social norms, rather than utility-maximising behaviour, and it allows us to analyse the issue of the persistence of class and exploitation abstracting from heterogeneous individual consumption behaviour. Unlike in many accumulation models in the Marxian tradition, however, the introduction of an (endogenously determined) subsistence bundle raises some interesting theoretical and technical issues, as it imposes a relevant and oft-neglected constraint on the set of equilibria.

\section{The basic model}

In this section, we set up and analyse the basic model, which is characterised by constant population, technology, preferences, consumption norms, and labour endowments over time. The focus on the basic model is motivated by analytical clarity and because it provides a theoretical benchmark and starting point for our analysis. However, the framework, concepts, and definitions presented in this section, and in the next one, can be easily extended and the results derived continue to hold in more general economies (as confirmed also by the simulations).

Let $\mathcal{N}_{t}=\mathcal{N}, \mathcal{P}_{t}=\mathcal{P}=\{(A, L)\}, b_{t}=b$, and $\left(l_{t-1}^{\nu}\right)_{\nu \in \mathcal{N}}=\left(l^{\nu}\right)_{\nu \in \mathcal{N}}$ for all $t$, and suppose that the economy is sufficiently productive to produce a surplus: $1-v b>0$, where $v=L(1-A)^{-1}$ denotes the embodied labour value. $^{2}$ In every $t$, given $\left(p_{t}, w_{t}\right)$, every agent $\nu \in \mathcal{N}$ chooses $x_{t}^{\nu}, y_{t}^{\nu}, z_{t}^{\nu}$, and $\delta_{t}^{\nu}$ to maximise her wealth subject to purchasing $b$ per unit of labour performed (1) and to the constraints set by her capital (2) and labour (3)

\footnotetext{
${ }^{2}$ The condition $1-v b>0$ is equivalent to $(1-b L)>A$ : it implies that if $A x$ units of capital are invested in the production process, $(1-b L) x>A x$ units of output (net of necessary consumption) are produced.
} 
endowments. Formally, every $\nu$ solves the following programme $M P_{t}^{\nu}$ :

$$
\max _{\left(x_{t}^{\nu} ; y_{t}^{\nu} ; z_{t}^{\nu} ; \delta_{t}^{\nu}\right)} p_{t} \omega_{t}^{\nu}
$$

subject to

$$
\begin{aligned}
p_{t} x_{t}^{\nu}+\left[p_{t}-w_{t} L\right] y_{t}^{\nu}+w_{t} z_{t}^{\nu}+p_{t} \delta_{t}^{\nu} & =p_{t} b \Lambda_{t}^{\nu}+p_{t} \omega_{t}^{\nu} \\
p_{t-1} A x_{t}^{\nu}+p_{t-1} A y_{t}^{\nu}+p_{t-1} \delta_{t}^{\nu} & =p_{t-1} \omega_{t-1}^{\nu}, \\
L x_{t}^{\nu}+z_{t}^{\nu} & \leqq l^{\nu} \\
x_{t}^{\nu}, y_{t}^{\nu}, z_{t}^{\nu}, \delta_{t}^{\nu}, \omega_{t}^{\nu} & \geqq 0 .
\end{aligned}
$$

Let $\mathcal{A}^{\nu}\left(p_{t}, w_{t}\right)$ be the set of actions $\xi_{t}^{\nu} \equiv\left(x_{t}^{\nu} ; y_{t}^{\nu} ; z_{t}^{\nu} ; \delta_{t}^{\nu}\right)$ that solve $M P_{t}^{\nu}$ at prices $\left(p_{t}, w_{t}\right)$. Let $V_{t}^{\nu}\left(p_{t-1} \omega_{t-1}^{\nu} ;\left(p_{t}, w_{t}\right)\right) \equiv \max p_{t} \omega_{t}^{\nu}$ be the value of $M P_{t}^{\nu}$ realised by the actions in $\mathcal{A}^{\nu}\left(p_{t}, w_{t}\right)$. Let $(p, w) \equiv\left\{\left(p_{t}, w_{t}\right)\right\}_{t=1, \ldots}$ and let $\left(x^{\nu} ; y^{\nu} ; z^{\nu} ; \delta^{\nu}\right) \equiv \xi^{\nu}=\left\{\xi_{t}^{\nu}\right\}_{t=1, \ldots}$. A basic accumulation economy is defined by the set of agents, $\mathcal{N}$, technology, $(A, L)$, consumption bundle, $b$, labour endowments, $\Pi$, and initial capital endowments, $\Omega_{0}$; and is denoted as $E\left(\mathcal{N} ;(A, L) ; b ; \Pi, \Omega_{0}\right)$, or, as a shorthand notation, $E_{0}$. Let $x_{t} \equiv \sum_{\nu \in \mathcal{N}} x_{t}^{\nu}$, and likewise for $y_{t}, z_{t}, \delta_{t}, \omega_{t}, \Lambda_{t}$, and $l$. Based on Roemer [14], the equilibrium notion can be defined.

Definition 1: A reproducible solution $(\mathrm{RS})$ for $E\left(\mathcal{N} ;(A, L) ; b ; \Pi, \Omega_{0}\right)$ is a vector $(p, w)$ and associated actions $\left(\xi^{\nu}\right)_{\nu \in \mathcal{N}}$, such that at all $t$ :

(a) $\xi_{t}^{\nu} \in \mathcal{A}^{\nu}\left(p_{t}, w_{t}\right)$, for all $\nu \in \mathcal{N}$ (individual optimality);

(b) $A\left(x_{t}+y_{t}\right)+\delta_{t} \leqq \omega_{t-1}$ (capital market);

(c) $L y_{t}=z_{t}$ (labour market);

(d) $\left(x_{t}+y_{t}\right)+\delta_{t} \geqq b \Lambda_{t}+\omega_{t}$ (goods market).

At a RS, in every period (a) all agents optimise; (b) aggregate capital is sufficient for production plans; (c) the labour market clears; (d) aggregate supply is sufficient for consumption and accumulation plans. The economy $E\left(\mathcal{N} ;(A, L) ; b ; \Pi, \Omega_{0}\right)$ can thus be interpreted either as a sequence of generations living for one period or as an infinitely-lived economy analysed in a sequence of temporary equilibria.

For any $(p, w)$, the profit rate at $t$ is $\pi_{t}=\frac{p_{t}-p_{t-1} A-w_{t} L}{p_{t-1} A}$. Given the structure of the economy, we shall focus on equilibria with strictly positive prices. ${ }^{3}$

\footnotetext{
${ }^{3}$ It immediately follows from $M P_{t}^{\nu}$ that if there is some $t^{\prime}$ such that $p_{t^{\prime}}=0$, then at any RS it must be $p_{t}=0$ for all $t>t^{\prime}$.
} 
Hence the profit rate is well defined at all $t$, and we can take the produced commodity as the numéraire, setting $p_{t}=1$, all $t$. Let the normalised price vector be denoted as $(\mathbf{1}, \widehat{w})$, where $\mathbf{1}=(1,1, \ldots)$ and, at any $t, \widehat{w}_{t}$ is the real wage rate. It is immediate to prove that at any $\mathrm{RS}$, if $\omega_{t-1}>0$, then $\widehat{w}_{t} \geqq b$ and $\pi_{t} \geqq 0$, all $t$.

Given the previous observations, by constraints (1)-(2), it follows that at any $\mathrm{RS}$, for all $\nu \in \mathcal{N}$ and all $t$, the following equation must hold

$$
\omega_{t}^{\nu}=\left[1-A-\widehat{w}_{t} L\right]\left(x_{t}^{\nu}+y_{t}^{\nu}\right)+\left(\widehat{w}_{t}-b\right)\left(L x_{t}^{\nu}+z_{t}^{\nu}\right)+\omega_{t-1}^{\nu} .
$$

Equation (5) has a number of implications. Lemma 1 proves that if the profit rate is strictly positive, then all wealth is used productively and if the wage rate is above subsistence, then the labour constraint (3) binds, for all agents at the solution to $M P_{t}^{\nu} \cdot{ }^{4}$

Lemma 1: Let $(1, \widehat{w})$ be a RS for $E_{0}$. At any $t$ : if $\pi_{t}>0$, then $A\left(x_{t}^{\nu}+y_{t}^{\nu}\right)=$ $\omega_{t-1}^{\nu}$, all $\nu \in \mathcal{N}$; and if $\widehat{w}_{t}>b$, then $L x_{t}^{\nu}+z_{t}^{\nu}=l^{\nu}$, all $\nu \in \mathcal{N}$.

Proof: By equation (5), if $\pi_{t}>0$, but $A\left(x_{t}^{\nu}+y_{t}^{\nu}\right)<\omega_{t-1}^{\nu}$, some $\nu \in \mathcal{N}$, then $\nu$ can increase $y_{t}^{\nu}$ and capital accumulation, contradicting optimality. Similarly, if $\widehat{w}_{t}>b$ and $L x_{t}^{\nu}+z_{t}^{\nu}<l^{\nu}, \nu \in \mathcal{N}$, then $\nu$ can increase $z_{t}^{\nu}$ and capital accumulation, contradicting optimality.

Next, it is possible to derive an explicit expression for the value of $M P_{t}^{\nu}$ and for the growth rate of capital, $g_{t}^{\nu}$, for all agents.

Lemma 2: Let $(\mathbf{1}, \widehat{w})$ be a RS for $E_{0}$. Then $V_{t}^{\nu}\left(\omega_{t-1}^{\nu} ;\left(1, \widehat{w}_{t}\right)\right)=\left(1+\pi_{t}\right) \omega_{t-1}^{\nu}+$ $\left(\widehat{w}_{t}-b\right) l^{\nu}$, and $g_{t}^{\nu}=\pi_{t}+\left(\widehat{w}_{t}-b\right) \frac{l^{\nu}}{\omega_{t-1}^{\nu}}$, for all $\nu \in \mathcal{N}$.

Proof: Straightforward from equation (5).

Lemma 2 has some interesting implications concerning the dynamics of accumulation. Let $\pi^{\max } \equiv \frac{1-A-b L}{A}$. Firstly, at all $t$, the aggregate growth rate of the economy is $g_{t}=\pi_{t}+\left(\widehat{w}_{t}-b\right) \frac{l}{\omega_{t-1}}$. Hence, if $l=L A^{-1} \omega_{t-1}$, then $g_{t}=\pi^{\max }$, and if $\widehat{w}_{t}=b$, then $g_{t}^{\nu}=g_{t}=\pi^{\max }$, for all $\nu \in \mathcal{N}$ such that $\omega_{t-1}^{\nu}>0$. Secondly, if $\widehat{w}_{t}>b$, then for any $\nu, \mu \in \mathcal{N}, g_{t}^{\nu}>g_{t}^{\mu}$ if and only if $\frac{l^{\nu}}{\omega_{t-1}^{\nu}}>\frac{l^{\mu}}{\omega_{t-1}^{\mu}}$. Finally, if $\pi_{t}=0$ then $g_{t}^{\nu}=\frac{(1-v b)}{v} \frac{l^{\nu}}{\omega_{t-1}^{\nu}}$, for all $\nu \in \mathcal{N}$ such that $\omega_{t-1}^{\nu}>0$, and $g_{t}=\frac{(1-v b)}{v} \frac{l}{\omega_{t-1}}$. Therefore, if there exists $t^{\prime} \geqq 1$ such that

\footnotetext{
${ }^{4}$ To be precise, a RS should be denoted as $\left((\mathbf{1}, \widehat{w}),\left(x^{\nu} ; y^{\nu} ; z^{\nu} ; \delta^{\nu}\right)_{\nu \in \mathcal{N}}\right)$. In what follows, we simply write $(\mathbf{1}, \widehat{w})$ for the sake of notational simplicity.
} 
$\pi_{t}=0$ for all $t \geqq t^{\prime}$, then the growth rate of the basic economy decreases over time and tends asymptotically to zero.

Lemma 3 derives a useful property of the set of solutions of $M P_{t}^{\nu}$.

Lemma 3: Let $(\mathbf{1}, \widehat{w})$ be a given price vector such that $\pi_{t} \geqq 0$ and $\widehat{w}_{t} \geqq$ $b$, some $t$. If $\xi_{t}^{\nu}$ solves $M P_{t}^{\nu}$, then $\xi_{t}^{\prime \nu} \in \mathbb{R}_{+}^{4}$ also solves $M P_{t}^{\nu}$ whenever $x_{t}^{\prime \nu}+y_{t}^{\prime \nu}=x_{t}^{\nu}+y_{t}^{\nu}$ and $z_{t}^{\prime \nu}-L y_{t}^{\prime \nu}=z_{t}^{\nu}-L y_{t}^{\nu}$.

Proof: It is easy to check that $\xi_{t}^{\prime \nu}$ satisfies constraints (1)-(2). Moreover, labour performed is the same in $\xi_{t}^{\nu}$ and $\xi_{t}^{\prime \nu}$, since $L\left(x_{t}^{\prime \nu}+y_{t}^{\prime \nu}\right)+\left(z_{t}^{\prime \nu}-L y_{t}^{\prime \nu}\right)=$ $L\left(x_{t}^{\nu}+y_{t}^{\nu}\right)+\left(z_{t}^{\nu}-L y_{t}^{\nu}\right)$. Then the result follows from equation (5).

Lemma 3 implies that if $\left(x_{t}^{\nu} ; y_{t}^{\nu} ; z_{t}^{\nu} ; \delta_{t}^{\nu}\right)$ solves $M P_{t}^{\nu}$, then there is another vector $\left(0 ; y_{t}^{\prime \nu} ; z_{t}^{\prime \nu} ; \delta_{t}^{\nu}\right)$ which solves $M P_{t}^{\nu}$. In the simulations, this allows us to select one of the many potential solutions of $M P_{t}^{\nu}$ by setting $x_{t}^{\nu}=0$ for all $\nu \in \mathcal{N}$.

Theorem 1 characterises the equilibria of the economy.

Theorem 1: Let $\left((\mathbf{1}, \widehat{w}),\left(\xi^{\nu}\right)_{\nu \in \mathcal{N}}\right)$ be a RS for $E_{0}$. At any $t$ :

(i) If $\pi_{t}>0$ and $\widehat{w}_{t}>b$, then $l=L A^{-1} \omega_{t-1}$. Furthermore, for any $\left(1, \widehat{w}_{t}^{\prime}\right)$ such that $\pi_{t}^{\prime} \geqq 0$ and $\widehat{w}_{t}^{\prime} \geqq b,\left(\xi_{t}^{\nu}\right)_{\nu \in \mathcal{N}}$ also satisfies conditions (a)-(d) of Definition 1;

(ii) If $l>L A^{-1} \omega_{t-1}>0$ then $\widehat{w}_{t}=b$;

(iii) If $l<L A^{-1} \omega_{t-1}$ then $\pi_{t}=0$.

Proof: Part (i). By Lemma 1, $A\left(x_{t}^{\nu}+y_{t}^{\nu}\right)=\omega_{t-1}^{\nu}$ and $L x_{t}^{\nu}+z_{t}^{\nu}=l^{\nu}$, for all $\nu \in \mathcal{N}$. Therefore, $A\left(x_{t}+y_{t}\right)=\omega_{t-1}$ and, by Definition $1(\mathrm{c}), L\left(x_{t}+y_{t}\right)=$ $L x_{t}+z_{t}=l$. Since $\left(x_{t}+y_{t}\right)=A^{-1} \omega_{t-1}$, we have $L\left(x_{t}+y_{t}\right)=L A^{-1} \omega_{t-1}=l$. To prove the second part of the statement, take any $\left(1, \widehat{w}_{t}^{\prime}\right)$ such that $\pi_{t}^{\prime} \geqq 0$ and $\widehat{w}_{t}^{\prime} \geqq b$. Then, it is immediate to show that $\xi_{t}^{\nu}$ solves $M P_{t}^{\nu}$ at $\left(1, \widehat{w}_{t}^{\prime}\right)$ for all $\nu$ and $\left(\xi_{t}^{\nu}\right)_{\nu \in \mathcal{N}}$ satisfies conditions (b)-(d) of Definition 1 by assumption.

Part (ii). At any RS, it must be $\widehat{w}_{t} \geqq b$. Suppose, contrary to the statement, that $\widehat{w}_{t}>b$. Then, for all $\nu \in \mathcal{N}$, by (2), $A\left(x_{t}^{\nu}+y_{t}^{\nu}\right) \leqq \omega_{t-1}^{\nu}$ and by Lemma $1, L x_{t}^{\nu}+z_{t}^{\nu}=l^{\nu}$. But, since $l>L A^{-1} \omega_{t-1}, L y_{t}<z_{t}$ holds, contradicting Definition 1(c). Therefore $\widehat{w}_{t}=b$.

Part (iii). At any RS, it must be $\pi_{t} \geqq 0$. Suppose, contrary to the statement, that $\pi_{t}>0$. Then, for all $\nu \in \mathcal{N}$, by (3), $L x_{t}^{\nu}+z_{t}^{\nu} \leqq l^{\nu}$ and by Lemma 1, $A\left(x_{t}^{\nu}+y_{t}^{\nu}\right)=\omega_{t-1}^{\nu}$. But, since $l<L A^{-1} \omega_{t-1}, L y_{t}>z_{t}$ holds, contradicting Definition $1(\mathrm{c})$. Therefore $\pi_{t}=0$. 
Theorem 1 defines the theoretical framework for the analysis of the dynamics of the economy and provides the foundations for the parameterisations of the price vector $(\mathbf{1}, \widehat{w})$. Although it only identifies necessary conditions for the existence of a RS, it does shed some light on how to construct the dynamic general equilibria of the economy. Consider part (ii) of the proof. Suppose $l>L A^{-1} \omega_{t-1}$, some $t$. If $\widehat{w}_{t}=b$, then $\pi_{t}>0$ and labour performed does not produce any net income for accumulation, and for all $\nu \in \mathcal{N}$, any $\left(0 ; y_{t}^{\nu} ; z_{t}^{\nu} ; 0\right)$ with $A y_{t}^{\nu}=\omega_{t-1}^{\nu}$ solves $M P_{t}^{\nu}$. Therefore since $A y_{t}=\omega_{t-1}$ and $l>L A^{-1} \omega_{t-1}$, we can choose a suitable profile $\left(z_{t}^{\nu}\right)_{\nu \in \mathcal{N}}$ such that $L y_{t}=z_{t}$ and all conditions of Definition 1 are satisfied at $t$.

Consider part (iii) of the proof. Suppose $l<L A^{-1} \omega_{t-1}$, some $t$. If $\pi_{t}=0$, then $\widehat{w}_{t}>b$ and capital holders are indifferent between using their wealth productively and just carrying it for sale at the end of the period, and for all $\nu \in \mathcal{N}$, any $\left(0 ; y_{t}^{\nu} ; z_{t}^{\nu} ; \delta_{t}^{\nu}\right)$ with $z_{t}^{\nu}=l^{\nu}$ solves $M P_{t}^{\nu}$. Therefore since $z_{t}=l$ and $l<L A^{-1} \omega_{t-1}$, we can choose a suitable profile $\left(y_{t}^{\nu}\right)_{\nu \in \mathcal{N}}$ such that $L y_{t}=z_{t}$ and all conditions of Definition 1 are satisfied at $t$.

\section{Exploitation and Class in the Accumula- tion Economy}

The concept of exploitation in the accumulation economy can now be introduced. In what follows, exploitation status is defined in every period $t$ : this is a natural assumption if the model describes a series of one-period economies, otherwise it reflects a focus on within period exploitation. ${ }^{5}$

Unlike in subsistence economies, focusing on the bundle consumed by an agent may be misleading as both poor and rich agents consume $b$ per unit of labour expended, but their potential consumption is very different. Definition 2 identifies exploitation status in terms of the bundles of goods that an agent can purchase with her income. More precisely, for all $\nu \in \mathcal{N}$ and all $\left(p_{t}, w_{t}\right)$, let $c_{t}^{\nu}$ satisfy $p_{t} c_{t}^{\nu}=V_{t}^{\nu}\left(W_{t-1}^{\nu} ;\left(p_{t}, w_{t}\right)\right)+p_{t} b \Lambda_{t}^{\nu}-p_{t} \omega_{t-1}^{\nu}$. Then

Definition 2 [Roemer [14]]: Agent $\nu$ is exploited at $t$ if and only if $\Lambda_{t}^{\nu}>v c_{t}^{\nu}$; she is an exploiter if and only if $\Lambda_{t}^{\nu}<v c_{t}^{\nu}$; and she is neither exploited nor an exploiter if and only if $\Lambda_{t}^{\nu}=v c_{t}^{\nu}$.

Theorem 2 characterises the exploitation status of every agent, based on their wealth per unit of labour performed $\frac{W_{t-1}^{\nu}}{\Lambda_{t}^{\nu}}$ :

\footnotetext{
${ }^{5}$ For a discussion of within period and whole life exploitation, see Veneziani $[17,18]$.
} 
Theorem 2: Let $(\mathbf{1}, \widehat{w})$ be a RS for $E_{0}$. At any $t$, if $\pi_{t}>0$ :

(i) agent $\nu$ is an exploiter $\Leftrightarrow \frac{W_{t-1}^{\nu}}{\Lambda_{t}^{\nu}}>\frac{1}{\pi_{t}} \frac{\left[1-\widehat{w}_{t} v\right]}{v}$;

(ii) agent $\nu$ is exploited $\Leftrightarrow \frac{W_{t-1}^{\nu}}{\Lambda_{t}^{\nu}}<\frac{1}{\pi_{t}} \frac{\left[1-\widehat{w}_{t} v\right]}{v}$;

(iii) agent $\nu$ is neither exploited nor an exploiter $\Leftrightarrow \frac{W_{t-1}^{\nu}}{\Lambda_{t}^{\nu}}=\frac{1}{\pi_{t}} \frac{\left[1-\widehat{w}_{t} v\right]}{v}$.

Proof: By Lemma 2, $V_{t}^{\nu}\left(W_{t-1}^{\nu} ;\left(1, \widehat{w}_{t}\right)\right)=\left(1+\pi_{t}\right) W_{t-1}^{\nu}+\left(\widehat{w}_{t}-b\right) l^{\nu}$, which in turn implies that $c_{t}^{\nu}=\pi_{t} W_{t-1}^{\nu}+\left(\widehat{w}_{t}-b\right) l^{\nu}+b \Lambda_{t}^{\nu}$, for all $t$ and all $\nu \in \mathcal{N}$. Therefore for any $\widehat{w}_{t} \geqq b$, using Lemma $1, c_{t}^{\nu}=\pi_{t} W_{t-1}^{\nu}+\widehat{w}_{t} \Lambda_{t}^{\nu}$, for all $t$ and all $\nu \in \mathcal{N}$. But then agent $\nu$ is an exploiter if and only if $v\left(\pi_{t} W_{t-1}^{\nu}+\widehat{w}_{t} \Lambda_{t}^{\nu}\right)>$ $\Lambda_{t}^{\nu}$, and the first part of the statement follows from simple algebraic manipulations. The other two parts follow in like manner.

Theorem 2 generalises analogous results by Roemer [14], as it characterises the exploitation status of all agents also in economies with unemployed labour. If $\widehat{w}_{t}>b$, then Lemma 1 implies that $\Lambda_{t}^{\nu}=l^{\nu}$, all $\nu \in \mathcal{N}$, and so by Theorem 2 exploitation status is determined by the ratio of capital and labour endowments as in Roemer [14]. However, by Theorem 1, if $l>L A^{-1} \omega_{t-1}$, then $\widehat{w}_{t}=b$ holds and Definition 1(c) implies that $\Lambda_{t}^{\nu}<l^{\nu}$ for at least some $\nu \in \mathcal{N}$. In this case, the economy is characterised by unemployed labour, and exploitation status is determined by the ratio of the capital endowment and labour performed, $\frac{W_{t-1}^{\nu}}{\Lambda_{t}^{\nu}}$.

Observe that Theorem 2 holds if $\pi_{t}>0$. If $\pi_{t}=0$ then $\widehat{w}_{t}=(1 / v)>b$ and $\Lambda_{t}^{\nu}=v c_{t}^{\nu}$ for all $\nu \in \mathcal{N}$ and no exploitation exists in the economy. This correspondence between profits and exploitation is a standard result in Marxian theory (for a discussion, see Veneziani and Yoshihara [19]).

Following Roemer [14], classes can be defined based on the way in which agents relate to the means of production. ${ }^{6}$ In every $t$, for any $\xi_{t}^{\nu} \in \mathcal{A}^{\nu}\left(p_{t}, w_{t}\right)$, consider only the first three entries of $\xi_{t}^{\nu}$, and let $\left(a_{1}, a_{2}, a_{3}\right)$ be a vector where $a_{i} \in\{+, 0\}, i=1,2,3$, where " + " means a positive entry. Agent $\nu$ is said to be a member of class $\left(a_{1}, a_{2}, a_{3}\right)$, if there is $\xi_{t}^{\nu} \in \mathcal{A}^{\nu}\left(p_{t}, w_{t}\right)$ such that $\left(x_{t}^{\nu} ; y_{t}^{\nu} ; z_{t}^{\nu}\right)$ has the form $\left(a_{1}, a_{2}, a_{3}\right)$. The notation $(+,+, 0)$ implies, for instance, that an agent works in her own 'shop' and hires others to work for her; $(+, 0,+)$ implies that an agent works both in her own 'shop' and for others; and so on. Although there are eight conceivable classes, only the following four are theoretically relevant.

\footnotetext{
${ }^{6}$ Again, observe that we focus on within period classes (see Veneziani [17, 18]).
} 
$C_{t}^{1}=\left\{\nu \in \mathcal{N} \mid \mathcal{A}^{\nu}\left(p_{t}, w_{t}\right)\right.$ has a solution of the form $\left.(+,+, 0) \backslash(+, 0,0)\right\}$,

$C_{t}^{2}=\left\{\nu \in \mathcal{N} \mid \mathcal{A}^{\nu}\left(p_{t}, w_{t}\right)\right.$ has a solution of the form $\left.(+, 0,0)\right\}$,

$C_{t}^{3}=\left\{\nu \in \mathcal{N} \mid \mathcal{A}^{\nu}\left(p_{t}, w_{t}\right)\right.$ has a solution of the form $\left.(+, 0,+) \backslash(+, 0,0)\right\}$,

$C_{t}^{4}=\left\{\nu \in \mathcal{N} \mid \mathcal{A}^{\nu}\left(p_{t}, w_{t}\right)\right.$ has a solution of the form $\left.(0,0,+)\right\}$.

The notation $\left(a_{1}, a_{2}, a_{3}\right) \backslash\left(a_{1}^{\prime}, a_{2}^{\prime}, a_{3}^{\prime}\right)$ means that agent $\nu$ is a member of class $\left(a_{1}, a_{2}, a_{3}\right)$ but not of class $\left(a_{1}^{\prime}, a_{2}^{\prime}, a_{3}^{\prime}\right)$.

To see that $C_{t}^{1}-C_{t}^{4}$ represent a partition of the set of agents, note first that classes $(0,+, 0)$ and $(0,0,0)$ can be ignored without loss of generality: at a RS with $\widehat{w}_{t}>b$ both classes are empty by Lemma 1 , while if $\widehat{w}_{t}=b$ (and so $\left.\pi_{t}>0\right)$ then agents in $(0,+, 0)$ and $(0,0,0)$ also belong to $(0,+,+)$ and $(0,0,+)$, respectively.

Theorem 3 characterises the class structure of the accumulating economy.

Theorem 3: Let $(\mathbf{1}, \widehat{w})$ be a RS for $E_{0}$. At any $t$, if $\pi_{t}>0$ :

(i) $\nu \in(+,+, 0) \backslash(+, 0,0) \Leftrightarrow L y_{t}^{\nu}>z_{t}^{\nu}$ for all $\xi_{t}^{\nu} \in \mathcal{A}^{\nu}\left(1, \widehat{w}_{t}\right)$;

(ii) $\nu \in(+, 0,0) \Leftrightarrow L y_{t}^{\nu}=z_{t}^{\nu}$ for some $\xi_{t}^{\nu} \in \mathcal{A}^{\nu}\left(1, \widehat{w}_{t}\right)$;

(iii) $\nu \in(+, 0,+) \backslash(+, 0,0) \Leftrightarrow L y_{t}^{\nu}<z_{t}^{\nu}$ for all $\xi_{t}^{\nu} \in \mathcal{A}^{\nu}\left(1, \widehat{w}_{t}\right)$;

(iv) $\nu \in(0,0,+) \Leftrightarrow W_{t-1}^{\nu}=0$.

Proof: 1. If $\pi_{t}>0$, then by Lemma 1, for all $\nu \in \mathcal{N}, A\left(x_{t}^{\nu}+y_{t}^{\nu}\right)=\omega_{t-1}^{\nu}$ for all $\xi_{t}^{\nu} \in \mathcal{A}^{\nu}\left(1, \widehat{w}_{t}\right)$. Therefore $\nu \in(0,0,+)$ implies $W_{t-1}^{\nu}=0$. Conversely, it is easy to see that for all $\widehat{w}_{t} \geqq b$, if $W_{t-1}^{\nu}=0$, then $\nu \in(0,0,+)$.

2. Consider agents with $W_{t-1}^{\nu}>0$. By the convexity of $M P_{t}^{\nu}$, if $L y_{t}^{\nu}>z_{t}^{\nu}$ for some $\xi_{t}^{\nu} \in \mathcal{A}^{\nu}\left(1, \widehat{w}_{t}\right)$ and $L y_{t}^{\prime \nu}<z_{t}^{\prime \nu}$ for some $\xi_{t}^{\prime \nu} \in \mathcal{A}^{\nu}\left(1, \widehat{w}_{t}\right)$, then there is $\xi_{t}^{\prime \prime \nu} \in \mathcal{A}^{\nu}\left(1, \widehat{w}_{t}\right)$ such that $L y_{t}^{\prime \prime \nu}=z_{t}^{\prime \prime \nu}$. Therefore, for all agents with $W_{t-1}^{\nu}>0$ : either $L y_{t}^{\nu}>z_{t}^{\nu}$ for all $\xi_{t}^{\nu} \in \mathcal{A}^{\nu}\left(1, \widehat{w}_{t}\right)$; or $L y_{t}^{\nu}<z_{t}^{\nu}$ for all $\xi_{t}^{\nu} \in \mathcal{A}^{\nu}\left(1, \widehat{w}_{t}\right)$; or $L y_{t}^{\nu}=z_{t}^{\nu}$ for some $\xi_{t}^{\nu} \in \mathcal{A}^{\nu}\left(1, \widehat{w}_{t}\right)$. The latter are mutually exclusive and exhaustive cases.

3. Part (i). Suppose $L y_{t}^{\nu}>z_{t}^{\nu}$ for all $\xi_{t}^{\nu} \in \mathcal{A}^{\nu}\left(1, \widehat{w}_{t}\right)$. We consider two cases.

Case 1: $\widehat{w}_{t}>b$. By Lemma 1, at all $\xi_{t}^{\nu} \in \mathcal{A}^{\nu}\left(1, \widehat{w}_{t}\right)$, it must be $A\left(x_{t}^{\nu}+y_{t}^{\nu}\right)=\omega_{t-1}^{\nu}$ and $L x_{t}^{\nu}+z_{t}^{\nu}=l^{\nu}$. From the first equation it follows that $L x_{t}^{\nu}+L y_{t}^{\nu}=L A^{-1} \omega_{t-1}^{\nu}$ and so we have $L A^{-1} \omega_{t-1}^{\nu}-L y_{t}^{\nu}=l^{\nu}-z_{t}^{\nu}$. Since $L y_{t}^{\nu}>z_{t}^{\nu}$ for all $\xi_{t}^{\nu} \in \mathcal{A}^{\nu}\left(1, \widehat{w}_{t}\right)$, then $L A^{-1} \omega_{t-1}^{\nu}>l^{\nu}$.

Consider $\xi_{t}^{\prime \nu}=\left(x_{t}^{\prime \nu} ; y_{t}^{\prime \nu} ; 0 ; 0\right)$ such that $L x_{t}^{\prime \nu}=l^{\nu}$, and $x_{t}^{\prime \nu}+y_{t}^{\prime \nu}=x_{t}^{\nu}+y_{t}^{\nu}$. Note that $y_{t}^{\prime \nu}=A^{-1} \omega_{t-1}^{\nu}-L^{-1} l^{\nu}>0$ and so noting that $z_{t}^{\prime \nu}-L y_{t}^{\prime \nu}=z_{t}^{\nu}-L y_{t}^{\nu}$, by Lemma 3 it follows that $\xi_{t}^{\prime \nu} \in \mathcal{A}^{\nu}\left(1, \widehat{w}_{t}\right)$. Hence, $\nu \in(+,+, 0)$. 
It remains to show that $\nu \notin(+, 0,0)$. Suppose, by way of contradiction, that there is $\xi_{t}^{\nu}=\left(x_{t}^{\nu} ; 0 ; 0 ; 0\right) \in \mathcal{A}^{\nu}\left(1, \widehat{w}_{t}\right)$. Since $\pi_{t}>0$, then by Lemma 1 , $A x_{t}^{\nu}=\omega_{t-1}^{\nu}$ and so $L x_{t}^{\nu}=L A^{-1} \omega_{t-1}^{\nu}>l^{\nu}$, a contradiction.

Case 2: $\widehat{w}_{t}=b$. In this case, any $\xi_{t}^{\nu}$ such that $\delta_{t}^{\nu}=0, A\left(x_{t}^{\nu}+y_{t}^{\nu}\right)=\omega_{t-1}^{\nu}$, and $L x_{t}^{\nu}+z_{t}^{\nu} \leqq l^{\nu}$, solves $M P_{t}^{\nu}$. Therefore, it is immediate to see that $\nu \in(+,+, 0)$. Further, $\xi_{t}^{\nu}=\left(0 ; A^{-1} \omega_{t-1}^{\nu} ; l^{\nu} ; 0\right) \in \mathcal{A}^{\nu}\left(1, \widehat{w}_{t}\right)$, and therefore $L y_{t}^{\nu}>z_{t}^{\nu}$ for all $\xi_{t}^{\nu} \in \mathcal{A}^{\nu}\left(1, \widehat{w}_{t}\right)$ implies $L A^{-1} \omega_{t-1}^{\nu}>l^{\nu}$. Hence the same argument as in Case 1 can be used to prove $\nu \notin(+, 0,0)$.

4. Parts (ii) and (iii) are proved similarly.

Theorem 3 provides a complete partition of the set of agents into classes, based on the way in which agents relate to the means of production. An immediate implication of Theorem 3 is that the class status of each agent is related to her productive endowments.

Corollary 1: Let $(\mathbf{1}, \widehat{w})$ be a RS for $E_{0}$. Consider any $t$, such that $\pi_{t}>0$. Then, $\nu \in C_{t}^{1} \Leftrightarrow L A^{-1} \omega_{t-1}^{\nu}>l^{\nu}$ and $\nu \in C_{t}^{4} \Leftrightarrow W_{t-1}^{\nu}=0$. Furthermore, if $\widehat{w}_{t}>b$, then $\nu \in C_{t}^{2} \Leftrightarrow L A^{-1} \omega_{t-1}^{\nu}=l^{\nu}$ and $\nu \in C_{t}^{3} \Leftrightarrow L A^{-1} \omega_{t-1}^{\nu}<l^{\nu}$; whereas if $\widehat{w}_{t}=b$, then $\nu \in C_{t}^{2} \Leftrightarrow L A^{-1} \omega_{t-1}^{\nu} \leqq l^{\nu}$ and $C_{t}^{3}=\varnothing$.

A fundamental insight of Marxian exploitation theory is the existence of a tight relation between class positions and exploitation status. This is formalised in the next principle. ${ }^{7}$

Class-Exploitation Correspondence Principle (CECP) [Roemer [14]]: Given any economy $E_{0}$, at any $R S$ and any $t$, if $\pi_{t}>0$ :

(A) every member of $C_{t}^{1}$ is an exploiter.

(B) every member of $C_{t}^{3} \cup C_{t}^{4}$ such that $\Lambda_{t}^{\nu}>0$ is exploited.

The next result proves that the CECP holds in the accumulation economies considered in this paper.

Theorem 4 (CECP): Let $(\mathbf{1}, \widehat{w})$ be a RS for $E_{0}$. At any $t$, such that $\pi_{t}>0$, if $\nu \in C_{t}^{1}$ then $\nu$ is an exploiter and if $\nu \in C_{t}^{3} \cup C_{t}^{4}$ then $\nu$ is exploited. Furthermore, if $\widehat{w}_{t}>b$ then:

$$
\begin{aligned}
& \nu \in C_{t}^{1} \Leftrightarrow \nu \text { is an exploiter; } \\
& \nu \in C_{t}^{2} \Leftrightarrow \nu \text { is neither exploited nor an exploiter; } \\
& \nu \in C_{t}^{3} \cup C_{t}^{4} \Leftrightarrow \nu \text { is exploited. }
\end{aligned}
$$

\footnotetext{
${ }^{7}$ In part (B), we impose the condition that agents in the lower classes spend some of their time working. This is a theoretically appropriate restriction since the exploitation status of agents who do not engage in any economic activities is unclear.
} 
Proof: 1. If $\widehat{w}_{t}>b$, then the result follows immediately from Corollary 1 and Theorem 2, noting that by Lemma $1, \Lambda_{t}^{\nu}=l^{\nu}$ and $\frac{1}{\pi_{t}} \frac{\left[1-\widehat{-}_{t} v\right]}{v}=\frac{A}{L}$.

2. Suppose that $\widehat{w}_{t}=b$. By Corollary $1, \nu \in C_{t}^{3} \cup C_{t}^{4} \Leftrightarrow W_{t-1}^{\nu}=0$. Hence by Theorem 2, if $\Lambda_{t}^{\nu}>0$, then $\nu$ is exploited. Further, by Corollary $1, \nu \in C_{t}^{1} \Leftrightarrow L A^{-1} \omega_{t-1}^{\nu}>l^{\nu}$. Noting that $\frac{W_{t-1}^{\nu}}{\Lambda_{t}^{\nu}} \geqq \frac{W_{t-1}^{\nu}}{l^{\nu}}>\frac{A}{L}=\frac{1}{\pi_{t}} \frac{\left[1-\widehat{w}_{t} v\right]}{v}$, the result follows from Theorem 2 .

Theorem 4 confirms the standard Marxist insight that agents in the upper classes are exploiters and agents in the lower classes are exploited.

\section{An index of exploitation}

The relation between class and exploitation status derived in the previous section provides some interesting normative insights on the structural injustices characterising capitalist economies, as Roemer [14] has forcefully argued. Yet, an exclusive focus on classes and on the sets of exploiters and exploited agents, as well as on the CECP, yields a rather partial, coarse picture of the structure of exploitative relations: two economies with similar numbers of agents belonging to each class and each exploitation category may still be very different. Based on Definition 2, it is possible to extend the normative reach of the concept of exploitation and provide a finer and more comprehensive picture of exploitative relations.

A first important characteristic of Definition 2 is that it allows us to move beyond a purely aggregate analysis and explore the exploitation status of every agent. This immediately raises the issue of the measurement of the intensity of exploitation, both at the individual and at the aggregate level. It is certainly desirable to have a notion of exploitation that allows us to make statements such as "agent A is less exploited than agent B", or "Economy C is more exploitative than economy D", or "Economy E is becoming increasingly exploitative over time".

Definition 2 states that exploitation status is determined according to whether $\Lambda_{t}^{\nu} \gtrless v c_{t}^{\nu}$. Therefore a natural index of the intensity of exploitation of any agent $\nu \in \mathcal{N}_{t}$ in period $t$ is:

$$
e_{t}^{\nu}=\frac{\Lambda_{t}^{\nu}}{v c_{t}^{\nu}}
$$

where $e_{t}^{\nu} \in[0, \infty)$, and $e_{t}^{\nu}$ can be interpreted as the rate of labour supplied relative to the labour necessary to obtain one unit of consumption. From this perspective, exploitative relations are equivalent to inequalities in 
labour hours supplied to earn one unit of income (measured in the labour numéraire) and the notion of exploitation is normatively relevant in that $e_{t}^{\nu}$ can be interpreted as a well-being index capturing access to some fundamental primary goods, namely economic resources (or commodities) and free hours. The profile $\left(e_{t}^{\nu}\right)_{\nu \in \mathcal{N}_{t}}$ then measures the distribution of access to resources and free hours and so of opportunity for well-being.

Agent $\nu$ is exploited if and only if $e_{t}^{\nu}>1$, but, assuming $e_{t}^{\nu}$ to be a meaningful cardinal and interpersonally comparable measure, a much richer analysis of exploitative relations is possible. For example, one can say that the greater $e_{t}^{\nu}$ the more exploited $\nu$ is and, for any $\nu, \mu \in \mathcal{N}_{t}$, if $e_{t}^{\nu}>e_{t}^{\mu}>1$ then $\nu$ is more exploited than $\mu$. And similarly for exploiters. More generally, we can analyse the distribution of $e_{t}^{\nu}$ in the economy at a given point in time, as well as its evolution over time, and ask questions about the exploitation structure of the economy. A more polarised distribution of $e_{t}^{\nu}$, for instance, suggests an increase in the intensity of exploitation. Interestingly, the mathematical structure of the profile $\left(e_{t}^{\nu}\right)_{\nu \in \mathcal{N}_{t}}$ is similar to that of income or wealth distributions, and so the measurement of some aggregate degree of exploitation raises similar issues as in the debate on the measurement of income or wealth inequalities. Below, we provide a complete description of the (dynamics of the) distribution of $e_{t}^{\nu}$. We also trace the evolution of the associated Gini index, interpreted as one example of a summary measure of the aggregate intensity of exploitation.

\section{The dynamics of the basic model}

This section analyses the basic model computationally. The aim is to illustrate the relevance of the theoretical results derived above and to rigorously describe the dynamics of exploitation and class - including the distribution of the exploitation intensity variable - in the benchmark case. This section also provides the basic formal and computational framework for the analysis of more complex economies below.

The simulation begins with data on $\left(\mathcal{N} ;(A, L) ; b ; \Pi, \Omega_{0}\right)$. The benchmark set of parameters is: $N=100, A=0.5, L=0.25, b=1.9$, and $l^{\nu}=$ 1 , for all $\nu \in \mathcal{N}$. As concerns productive assets, aggregate initial capital is $\omega_{0}=25$. This guarantees that $l>L A^{-1} \omega_{0}$ holds and the economy is initially capital constrained, starting far from the knife-edge condition $l=$ $L A^{-1} \omega_{t-1}$. This is important in order to examine the existence and evolution of exploitation, profits and classes over time: by Theorem 1, the knife-edge condition identifies a ceiling to capital accumulation as it provides an upper 
bound to the aggregate amount of capital compatible with the existence of positive profits and exploitation.

The distribution of aggregate capital is determined in order to mimic the empirical wealth distribution in the U.S. economy as reported by Allegretto [1]. In all of our simulations, $\omega_{0}$ is distributed such that there are five groups of agents. The first group comprises $50-70 \%$ of the total population and each $\nu$ in this group is assigned $\omega_{0}^{\nu}=0 .{ }^{8}$ The top $1 \%$ of agents are assigned $40 \%$ of $\omega_{0}$, the next $4 \%$ are assigned $30 \%$ of $\omega_{0}$, the next $15 \%$ are assigned $20 \%$, and the remaining $10 \%$ of $\omega_{0}$ is distributed to whatever agents remain (minimum $10 \%$ and maximum $30 \%$ of $N$ ).

This parameterisation represents our benchmark in all simulations, unless otherwise stated. ${ }^{9}$ It is worth stressing at the outset that the values chosen are empirically reasonable, but - as shown in section 9 below - our key insights are robust to different choices of the initial values of the key parameters.

Concerning agents, by Lemma 3, we restrict the computational analysis to solutions of $M P_{t}^{\nu}$ for all $\nu$ and $t$ of the form $\left(0 ; y_{t}^{\nu} ; z_{t}^{\nu} ; \delta_{t}^{\nu}\right)$. To be specific, at any $t$, we set $\xi_{t}^{\nu}=\left(0 ; A^{-1} \omega_{t-1}^{\nu} ; \frac{L A^{-1} \omega_{t-1}}{l} l^{\nu} ; 0\right), \xi_{t}^{\nu}=\left(0 ; \frac{l}{L A^{-1} \omega_{t-1}} A^{-1} \omega_{t-1}^{\nu} ; l^{\nu}\right.$; $\left.\left(1-\frac{l}{L A^{-1} \omega_{t-1}}\right) \omega_{t-1}^{\nu}\right)$, or $\xi_{t}^{\nu}=\left(0 ; A^{-1} \omega_{t-1}^{\nu} ; l^{\nu} ; 0\right)$, for all $\nu$, depending on whether the economy is capital constrained, labour constrained, or on the knife-edge. In all of our simulations, this specification of agents' optimal choices guarantees that the conditions in Definition 1 are always satisfied. ${ }^{10}$

The simulation runs for $T=50$ periods. The simulation first checks whether the economy is capital constrained, labour constrained, or on the knife-edge and updates $\widehat{w}_{t}$ accordingly. Given the choice of $\omega_{0}$, the simulation begins with $\widehat{w}_{1}=b$. Once $\widehat{w}_{t}$ is determined, $\pi_{t}$ is known and the agents then solve $M P_{t}^{\nu}$. The agent's endowments are updated according to equation (5) and the simulation then repeats as necessary. ${ }^{11}$

The results of the simulation over $T$ can be found in Figures 1-3. Figure 1 reports the aggregate activity levels $\left(x_{t}, y_{t}, z_{t}, \delta_{t}\right)$, aggregate net output $(1-A) y_{t}$, net output per capita $(1-A) y_{t} / N$, wealth $W_{t-1}$, the growth rate of capital $g_{t}, \widehat{w}_{t}$ and $b$, and $\pi_{t}$. In all panels, the dashed vertical line denotes

\footnotetext{
${ }^{8}$ The actual number of agents assigned to this first group is randomly drawn from a uniform distribution.

${ }^{9}$ The procedure to determine the initial wealth distribution is the same in all versions of the model. While the initial distributions will inevitably differ across models due to different starting points in relation to the knife-edge and to the randomness built into the procedure, the differences are sufficiently small that the results of the simulations are unaffected and can be compared across models.

${ }^{10}$ See Section A.1 of the Appendix.

${ }^{11}$ All simulations are done using Mathematica version 10.
} 
the period in which the economy becomes labour constrained.

Figure 2(a) reports the dynamics of exploitation by providing a headcount of the agents who are exploiters, exploited, or neither according to Definition 2. Clearly, the exploitation status of agents is constant while the economy is capital constrained and exploitation ceases to exist once it becomes labour constrained.

Figure 2(b) captures the class composition of the economy. The pattern of $C_{t}^{1}$ and $C_{t}^{2}$ is interesting and it reveals the relation between endowments and class status: at the beginning of the simulation, a relatively low level of capital implies that for a number of agents labour demand $\left(L y_{t}^{\nu}\right)$ is lower than their labour endowment $\left(l^{\nu}\right)$, placing them into $C_{t}^{2}$. As accumulation progresses, however, so does their labour demand and these agents eventually join $C_{t}^{1} .^{12}$

Figure 2(c) illustrates Theorem 4 and shows the existence of robust correspondence between class and exploitation status (until the economy becomes labour constrained).

Figure 3 provides a complete description of the distribution of the exploitation intensity index, $e_{t}^{\nu}$, over the course of the simulation. Prior to the economy becoming labour constrained, the distribution of $e_{t}^{\nu}$ is constant over time: there is no tendency for exploitation to diminish. When the economy becomes labour constrained, profits and exploitation disappear, and one can observe that $e_{t}^{\nu}=1$, all $\nu \in \mathcal{N}$. Interestingly, agents in the lower classes are exploited to somewhat different degrees. All agents in $C_{t}^{4}$ are exploited with equal intensity, since they all have zero endowments and work the same amount. The exploitation status of agents in $C_{t}^{2}$ is not obvious instead: some of them are indeed exploited (albeit less than members of $C_{t}^{4}$ ) but agents at the upper end of $C_{t}^{2}$ are exploiters (albeit less than members of $C_{t}^{1}$ ).

Figure 3 displays a relatively low dispersion of the exploitation index. This is due to the fact that, unlike in actual economies, all agents perform the same amount of labour and, in the capital constrained phase, the given parameterisation (in particular the rather high value of $b$ ) yields a low profit rate. Different values of the parameters, or a heterogeneous allocation of labour (perhaps inversely proportional to wealth, in order to reflect class differences) lead to a much higher dispersion (see section 9 below). ${ }^{13}$

In summary, the results of the basic model confirm that exploitation and

\footnotetext{
${ }^{12}$ In Figure 2(b), and in all similar figures below, the class composition of the economy is shown only for the periods $t$ with $\pi_{t}>0$. For if the profit rate vanishes Theorem 3 and Corollary 1 no longer apply, and the definition of classes needs to be revised.

${ }^{13}$ The Gini coefficient of $\left(e_{t}^{\nu}\right)_{v \in \mathcal{N}}$ is constant and equal to 0.0281395 until the economy becomes labour constrained, when it drops to zero.
} 
Figure 1: Summary results - Basic model
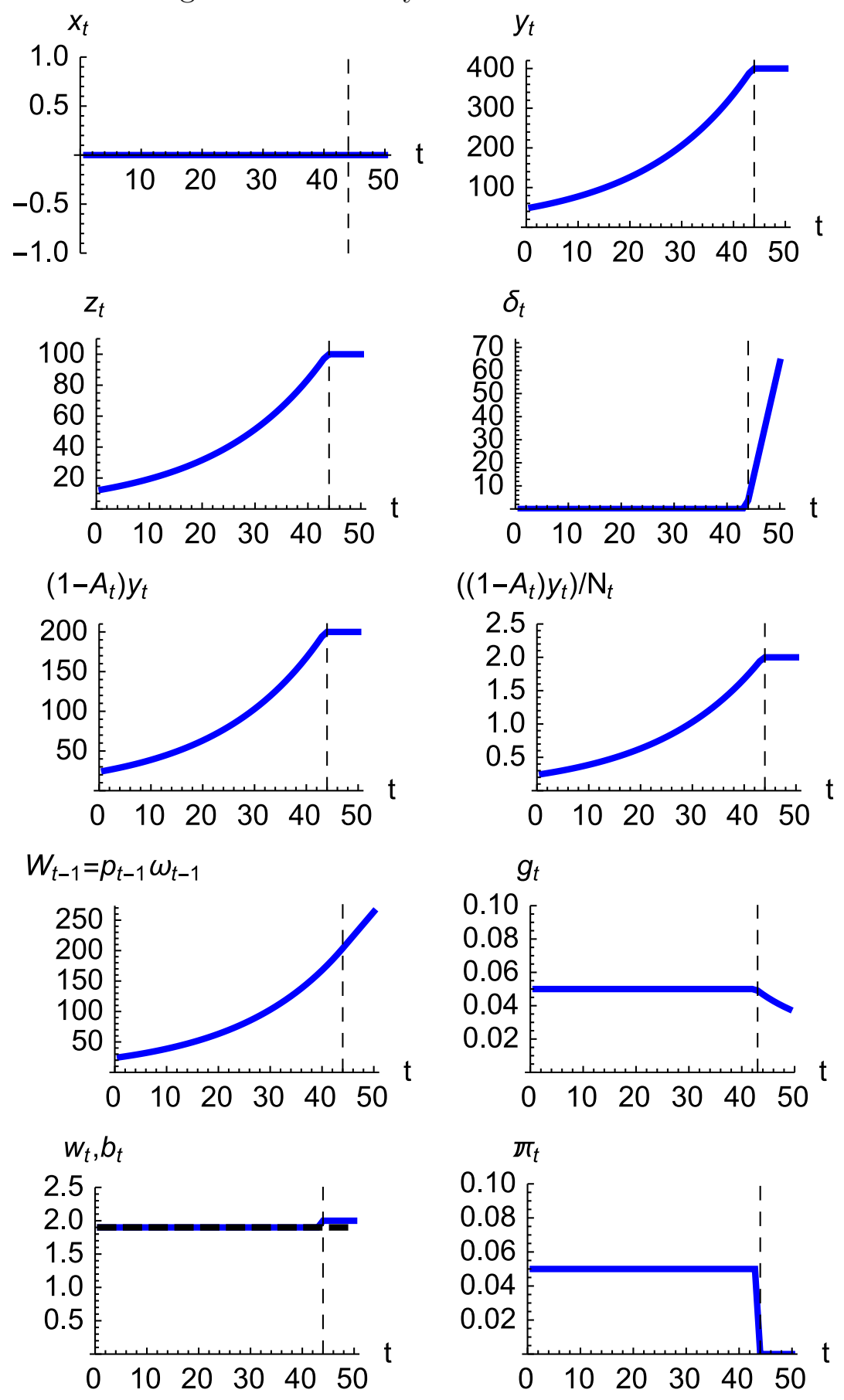
Figure 2: Class and exploitation status - Basic model

(a) Exploitation status

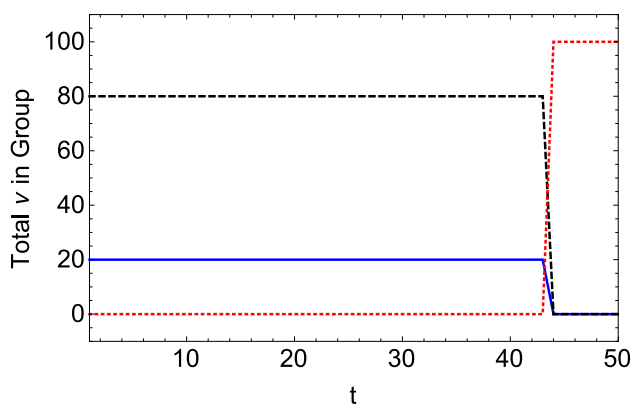

(b) Class status

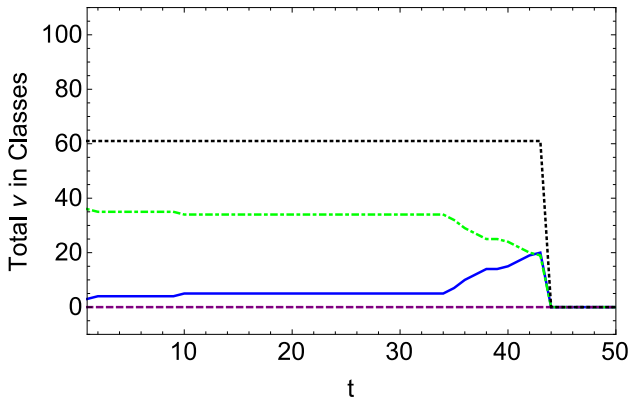

(c) $\mathrm{CECP}$

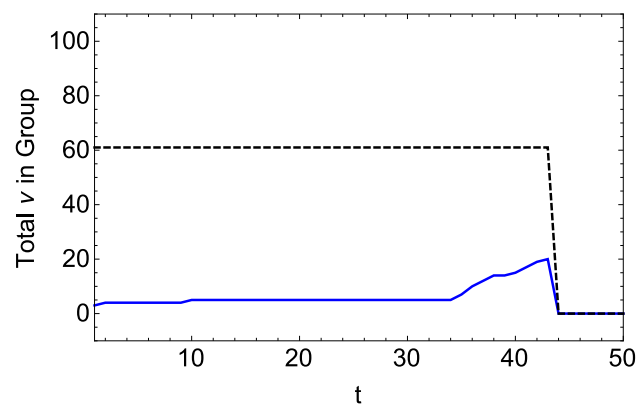

$\sum v \in \mathrm{C}_{\mathrm{t}}^{1} \wedge$ Exploiter $\quad$-.-. $\sum v \in\left(\mathrm{C}_{\mathrm{t}}^{3} \cup \mathrm{C}_{\mathrm{t}}^{4}\right) \wedge$ Exploited

classes are meaningful concepts to analyse the economic and social structure of capitalist economies, and the distribution of exploitation intensity index yields interesting normative insights. They also confirm, however, that in an accumulation economy, absent any countervailing forces, exploitation disappears over time. Our next step, therefore, is to extend the basic model in order to explore possible mechanisms that could lead to the persistence of exploitation in the presence of accumulation.

In all of the following extensions, we introduce technical change as a key mechanism for maintaining labour abundant relative to capital. Technical change plays a prominent role in Marxian theory and it has been central in debates on the persistence of exploitation (e.g. Skillman [15]). But we consider two different models to determine the key distributive variables, 
Figure 3: Exploitation intensity index - Basic model

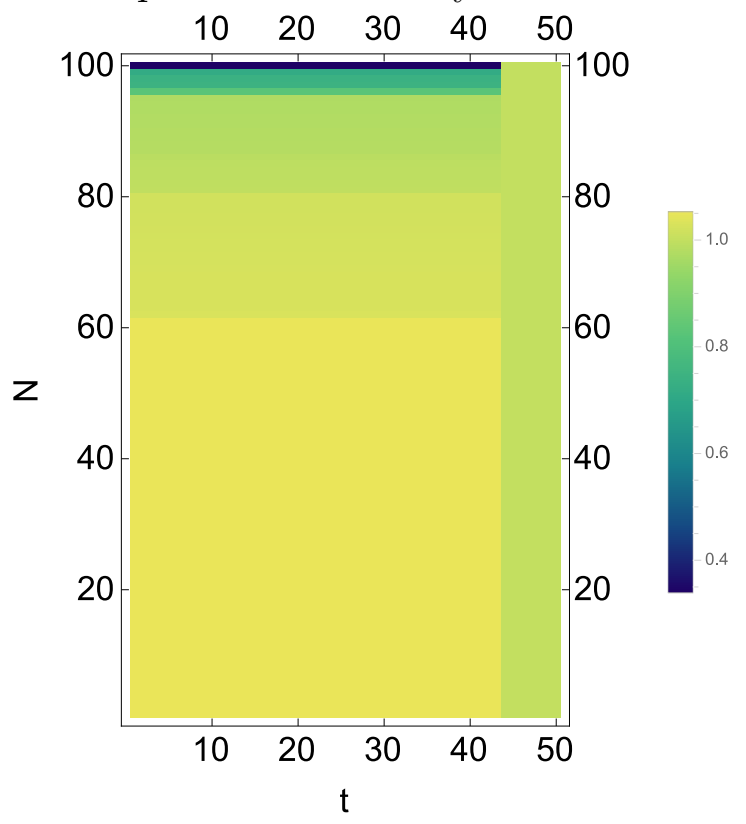

namely wages and profits. In the next section, we explore the interplay of technical change and the evolution of social norms, whereas in section 8 we set up a general bargaining model.

\section{Endogenising consumption and technical change}

In this section we extend the basic model to allow both the consumption bundle, $b_{t}$, and the production technology, $\left(A_{t}, L_{t}\right)$ to be determined endogenously and change over time, and analyse their effect on the dynamics of exploitation and classes. This choice reflects both empirical and theoretical concerns. Empirically, the long-run evolution of capitalist economies has indeed been characterised by an increase in (average) consumption opportunities and by a tendential expansion of technical knowledge, leading to a progressive increase in labour productivity (see Flaschel et al. [7]).

Theoretically, a fundamental feature of capitalism as a dynamic system is its constant tendency to revolutionise production. It is certainly restrictive to assume the production technology $(A, L)$ to remain constant over time, and regardless of changes in prices and distribution. Labour-saving technical progress may also play a crucial role in the dynamics of exploitation by guaranteeing the persistent abundance of labour. Further, although sub- 
jective consumption choices are not central in the Marxian analysis of class and exploitation, it is restrictive (and unrealistic) to assume that consumption norms remain constant over time with the subsistence wage at some biologically-determined level.

To be specific, concerning consumption, we incorporate some Marxian insights on the social nature of consumption and assume that $b_{t}$ is the product of social norms, by making it a function of the general level of development of the economy, as proxied by aggregate capital, and of the history of consumption itself. Formally,

$$
b_{t}=f_{1}\left(b_{t-1}, \omega_{t-2}, \omega_{t-1}\right) .
$$

With the specification used in the simulation as follows:

$$
b_{t}=b_{t-1} \cdot\left(1+\phi \frac{\omega_{t-1}-\omega_{t-2}}{\omega_{t-2}}\right),
$$

where the parameter $\phi$ captures the degree to which the degree of development of the economy influences consumption norms.

Concerning technology, we follow Marx and assume that when the profit rate falls beneath a certain threshold, capitalists increase their efforts to innovate and introduce new capital-using labour-saving techniques. Formally,

$$
\left(A_{t}, L_{t}\right)=f_{2}\left(\widehat{w}_{t} ; A_{t-1}, L_{t-1}\right)
$$

where $f_{2}$ is defined as follows

$$
\begin{aligned}
\left(A_{t}, L_{t}\right) & =\left(A_{t-1}, L_{t-1}\right), \text { if } \pi^{\left(\widehat{w}_{t} ; A_{t-1}, L_{t-1}\right)}=\frac{1-A_{t-1}-\widehat{w}_{t} L_{t-1}}{A_{t-1}} \geqq \pi^{*}, \\
\left(A_{t}, L_{t}\right) & =\left(A^{\prime}, L^{\prime}\right), \text { if } \pi^{\left(\widehat{w}_{t} ; A_{t-1}, L_{t-1}\right)}=\frac{1-A_{t-1}-\widehat{w}_{t} L_{t-1}}{A_{t-1}}<\pi^{*},
\end{aligned}
$$

where $\pi^{*}$ is the capitalist's minimum profitability benchmark, which will depend on economic, institutional and even cultural factors. The new technique $\left(A^{\prime}, L^{\prime}\right)$ is chosen such that $A^{\prime} \geqq A_{t-1}, L^{\prime}<L_{t-1}$, and $\pi^{\prime}=\frac{1-A^{\prime}-\widehat{w}_{t} L^{\prime}}{A^{\prime}}>$ $\pi^{\left(\widehat{w}_{t} ; A_{t-1}, L_{t-1}\right)}$. In the simulations, we assume that capitalists decide whether to introduce new techniques as soon as they know $b_{t}$ and the real wage $\widehat{w}_{t}$ and thus $\pi^{\left(\widehat{w}_{t} ; A_{t-1}, L_{t-1}\right)}$. This is both theoretically and empirically reasonable, but it is worth stressing that - as argued in section 9 below - the main results are robust to alternative assumptions on the timing of innovations. 


\subsection{Simulation results}

In our simulations, we set $\pi^{*}=0.01$. When $\pi^{\left(\widehat{w}_{t} ; A_{t-1}, L_{t-1}\right)}<\pi^{*}$, the new technique prevailing at $t$ is identified by first selecting a profit rate, $\pi^{\prime}$, from the set of all previous profit rates $\left\{\pi_{\tau}\right\}_{\tau<t}$, such that $\pi_{\tau}>\pi^{*}$ and then randomly choosing an increase in $A_{t-1}$ in the range [0.01,0.03] and setting $L_{t}=\frac{1-A_{t}-A_{t} \pi^{\prime}}{\widehat{w}_{t}}$. To ensure that $A_{t}<1$ a limit is set such that $A_{\max }=0.991$. In the event that the pseudo-randomly determined $\pi^{\prime}$ and $A_{t}$ entail a negative $L_{t}, \pi^{\prime}$ is adjusted downward by 0.02 so that $L_{t}>0$.

The simulation occurs in the following order: (1) initialisation, $t=1$; (2) subsistence $b_{t}$ is updated; (3) $\widehat{w}_{t}$ and $\pi^{\left(\widehat{w}_{t} ; A_{t-1}, L_{t-1}\right)}$ are determined; ${ }^{14}$ (4) given $\pi^{\left(\widehat{w}_{t} ; A_{t-1}, L_{t-1}\right)}, A_{t}$ and $L_{t}$ are updated if appropriate, leading $\pi_{t}$ to be subsequently updated to reflect the new technology; (5) agents solve $M P_{t}^{\nu}$; and (6) the sequence (2)-(5) is repeated for $T$.

The simulation runs for $T=50$, with $A_{0}=0.5, L_{0}=1, b_{0}=0.3$, $\phi=1, l_{t}^{\nu}=1$ for all $\nu \in \mathcal{N}_{t}$ and all $t$, and $N_{t}=100$, all $t$. The initial distribution of endowments $\Omega_{0}$ is chosen as in the basic model, with aggregate initial endowments $\omega_{0}=15$, so that $l>L_{1} A_{1}^{-1} \omega_{0}$ and the model is capital constrained at the start of the simulation.

The main results of the model are depicted in Figures 4-7(b). Figure 4 reports the same information as Figure 1 for the basic model. Some differences between Figures 1 and 4 clearly emerge, notably concerning the dynamics of net output, wealth, and labour performed. While net output and wealth grow over the course of the simulation (as driven by $y_{t}$ ), $z_{t}$ exhibits a cyclical downward trend. This is caused by the decreasing trend of $L_{t}$ together with its cyclical movements driven by the periods of accumulation and rising labour demand occurring between (labour-saving) technological changes. Throughout the cycles of accumulation and technical change the economy remains capital constrained. Therefore there is a secular increase in $\widehat{w}_{t}=b_{t}$ from $b_{0}=0.3$ to $b_{\max }=92.6886$, and, as expected, $\pi_{t}$ exhibits cyclical behaviour in the range of its initial value $\pi_{0}=0.4$ and a minimum of $\pi_{\min }=0.0100257$ (see Figure 4). Figure 5 shows the dynamics of $A_{t}, L_{t}$, and labour values. ${ }^{15}$

The interaction among consumption behaviour, social norms and capitalist innovations leads to a very interesting dynamic behaviour. As predicted, technical change allows exploitation and classes to persist. The intuition

\footnotetext{
${ }^{14}$ The determination of the wage rate and profit rate depends on whether the economy is capital constrained, labour constrained, or on the knife-edge. If the economy is capital constrained we set $\widehat{w}_{t}=b_{t}$.

${ }^{15}$ The condition $v_{t} b_{t}<1$ holds throughout the simulation even as $\left(v_{t}, b_{t}\right)$ change over $T$.
} 
Figure 4: Summary results - Model with endogenous subsistence and technical change
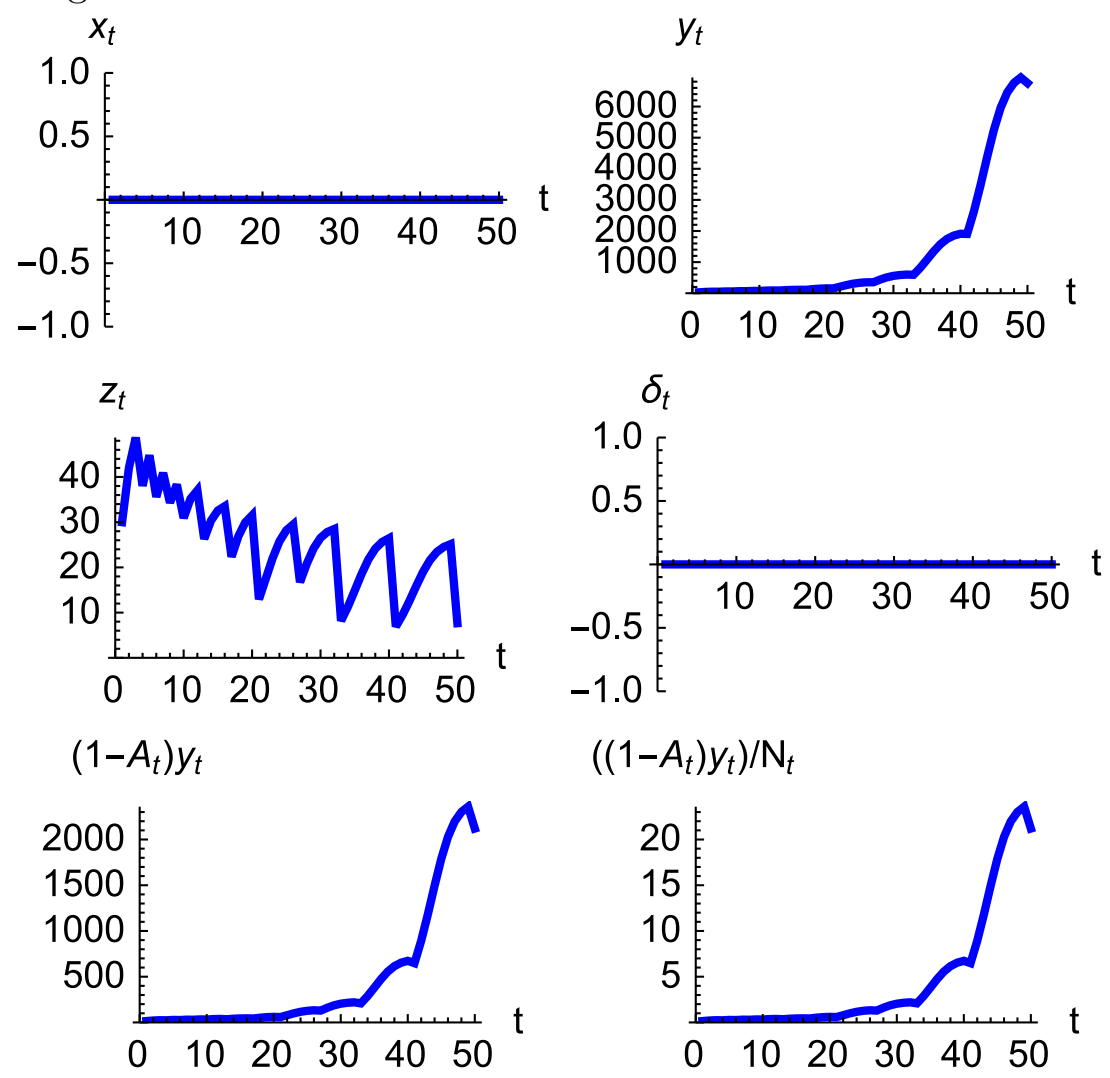

$\left(\left(1-A_{t}\right) y_{t}\right) / \mathrm{N}_{t}$

$W_{t-1}=p_{t-1} \omega_{t-1}$
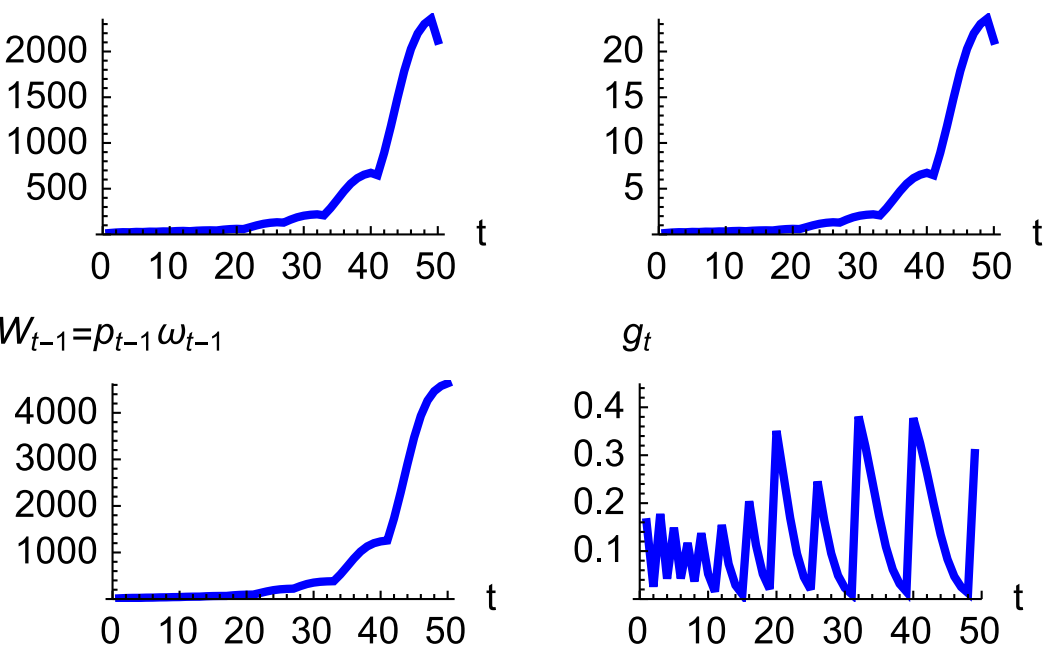

$g_{t}$
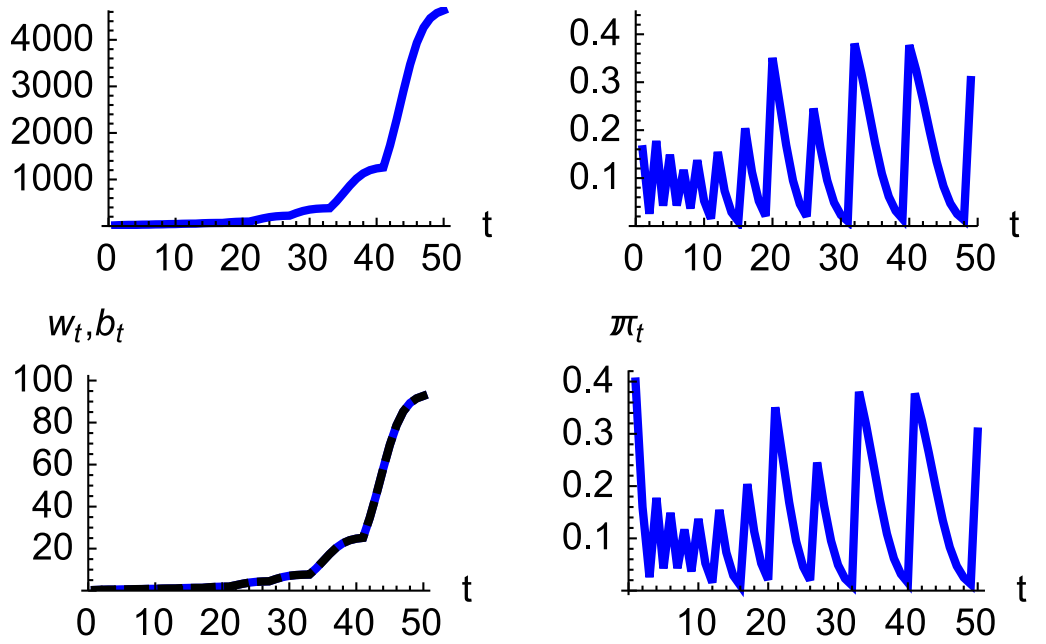
Figure 5: Technology and labour values - Model with endogenous subsistence and technical change
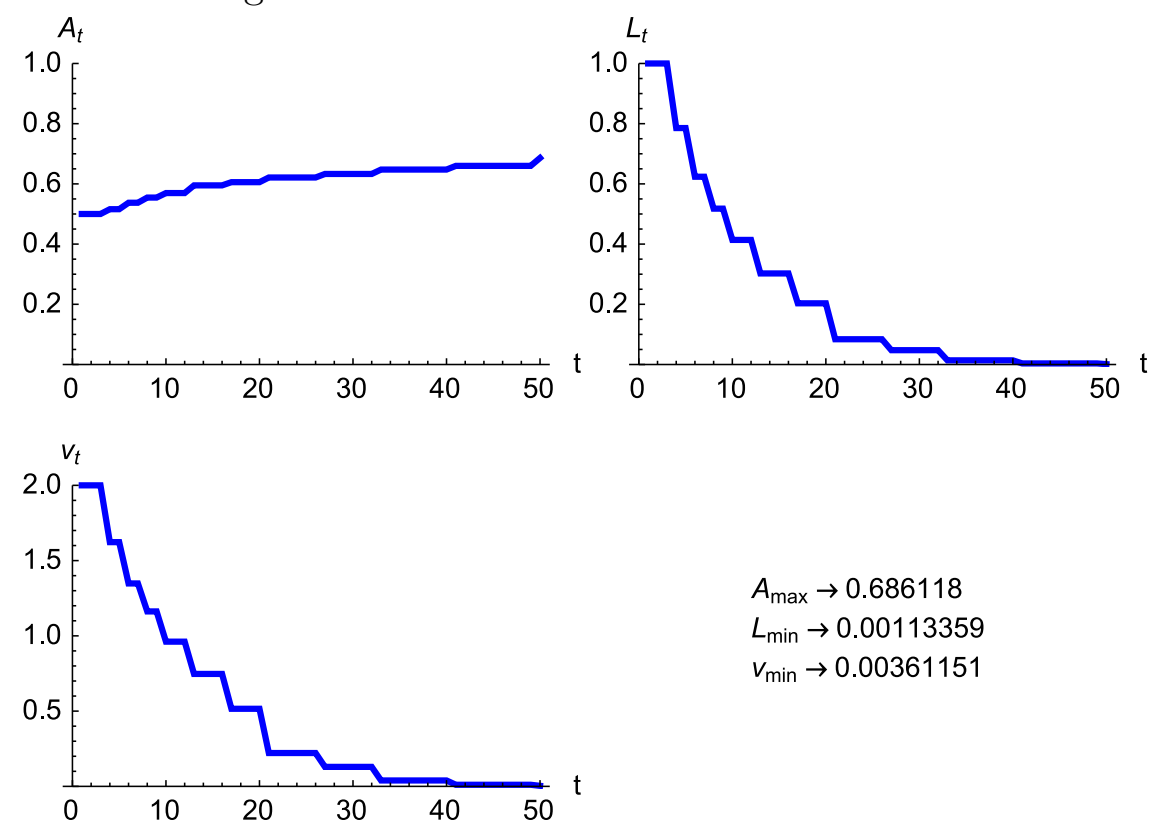

$A_{\max } \rightarrow 0.686118$

$L_{\min } \rightarrow 0.00113359$

$v_{\min } \rightarrow 0.00361151$

is the following. Since the economy is initially capital constrained, then $\widehat{w}_{t}=b_{t}$ for at least some periods $t, t \geq 1$. As accumulation proceeds, the subsistence norm, $b_{t}$, increases and so does the real wage rate in equilibrium. Therefore the profit rate starts declining before the economy becomes labour constrained. But as the profit rate goes beneath $\pi^{*}$, an innovation is introduced which restores profitability, and so on. It is noteworthy that a strongly cyclical behaviour is generated in an essentially linear economy: it is the interaction of technical change and capitalist behaviour that generates persistent growth cycles.

Figures 6(a)-6(b) show the remarkable persistence and stability of the basic exploitation and class structure of the economy produced by the introduction of technical change. ${ }^{16}$ Figure $6(\mathrm{c})$ confirms that the CECP continues to hold in the more general economy considered here.

Figures 6(a)-6(c) focus on the basic exploitation and class structure of the economy, as in the standard Marxian approach, and thus draw a partial picture of exploitative relations. To see this, consider Figures 7(a)-7(b) which provide information about the distribution of the exploitation intensity in-

\footnotetext{
${ }^{16}$ The "bumps" in the number of agents in $C_{t}^{1}$ and $C_{t}^{2}$ in Figure $6(\mathrm{~b})$ are due to decreases in $L_{t}$ yielding $L_{t} y_{t}^{\nu} \leq l^{\nu}$ for some $\nu$ who were previously members of $C_{t}^{1}$.
} 
Figure 6: Class and exploitation status - Model with endogenous subsistence and technical change

(a) Exploitation status

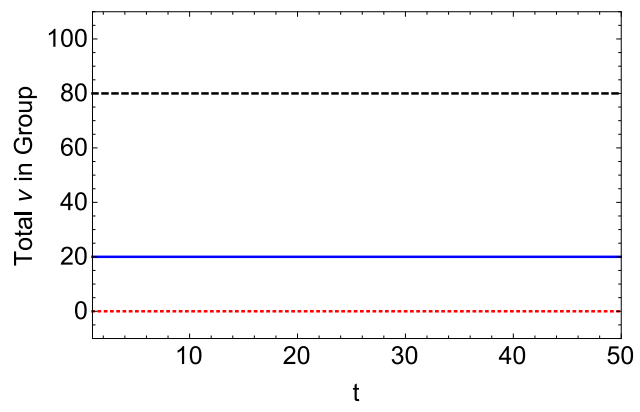

\begin{tabular}{ll} 
- Exploiters $\quad \ldots . .$. & Neither Exploiter or Exploited \\
\hline- Exploited & \\
\hline
\end{tabular}

(b) Class status

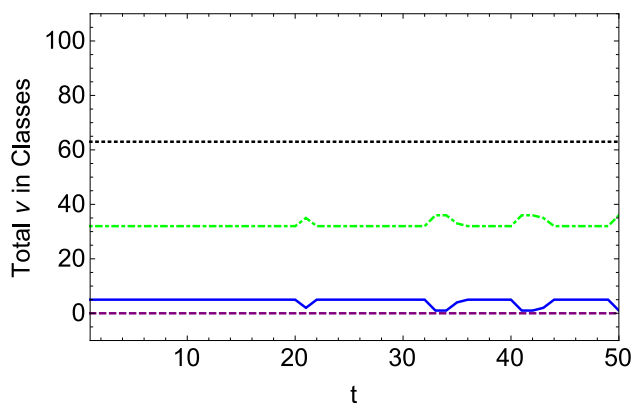

$\begin{aligned}-\mathrm{C}_{\mathrm{t}}^{1} & =\left\{\sum v \in(+,+, 0) \backslash(+, 0,0) ; \mathrm{L}_{\mathrm{t}} \mathrm{y}_{\mathrm{t}}^{v}>\mathrm{l}^{v}\right\} \\ \cdots--\mathrm{C}_{\mathrm{t}}^{2} & =\left\{\sum v \in(+, 0,0) ; \mathrm{L}_{\mathrm{t}} \mathrm{y}_{\mathrm{t}}^{v} \leq 1^{v}\right\} \\ \cdots \mathrm{C}_{\mathrm{t}}^{3} & =\phi \\ \cdots \mathrm{C}_{\mathrm{t}}^{4} & =\left\{\sum v \in(0,0,+) ; \mathrm{W}_{\mathrm{t}-1}^{v}=0\right\}\end{aligned}$

(c) CECP

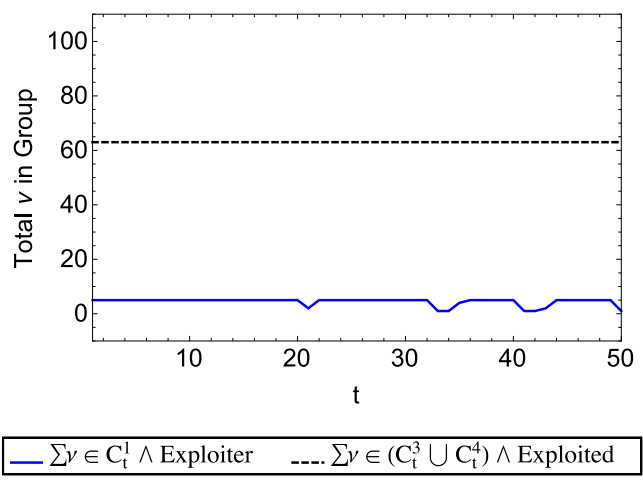

dex $e_{t}^{\nu}$. Under the surface of a seemingly unchanging pattern of exploitation, Figures 7(a)-7(b) show that the addition of technical change induces an interesting phenomenon of "exploitation cycles". As accumulation progresses with a given technique $\left(A_{t}, L_{t}\right)$, exploitation tends to decrease as $e_{t}^{\nu}$ tends to 1 for all $\nu$. However, when a new technique is introduced, profitability and inequality in exploitation intensity are restored, driving a wedge between the lower and upper classes, and the pattern of accumulation and exploitation resumes until another production technique is introduced.

The results support the claim that capital-using labour-saving technical change can help to explain the persistence of exploitation in accumulation economies (Skillman [15]). The key role of technical change in this context is 
Figure 7: Exploitation intensity index - Model with endogenous subsistence and technical change

(a) Exploitation intensity across agents

(b) Gini coefficient of $e_{t}^{\nu}$
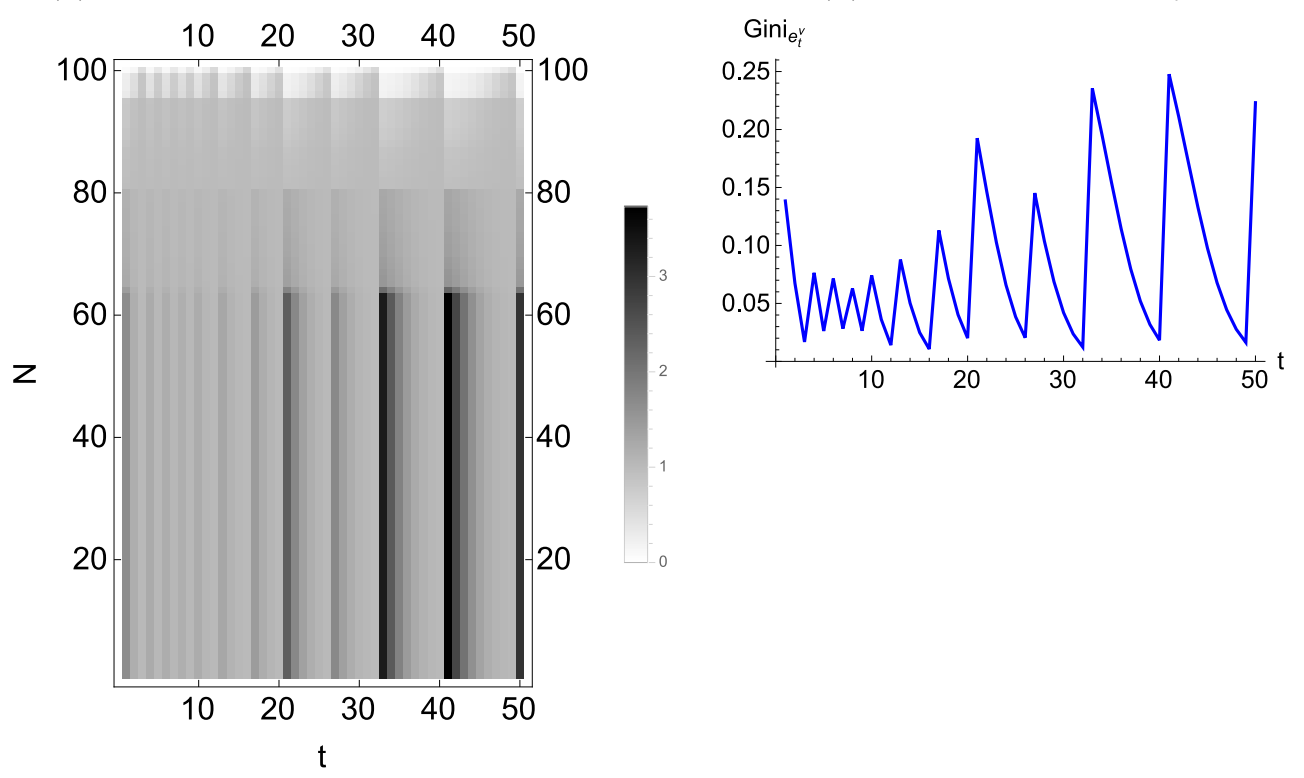

to make capital persistently scarce relative to labour and to maintain labour unemployment throughout. This is certainly an important insight but it is worth stressing that it derives from a rather specific mechanism to determine distributive variables: technical change plays a crucial role in creating labour unemployment which in turn forces the real wage down to the (socially determined and time-evolving) subsistence level. This immediately raises two issues. First, it appears that labour unemployment is a necessary determinant of the persistence of exploitative relations. Yet it would be important, both normatively and theoretically, to analyse exploitation and class in economies with full employment. Nothing in Marx's theory of exploitation and class depends on the existence of unemployment and full employment does not make capitalist economies non-exploitative. Second, given the extremely skewed asset distribution, the assumption of a perfectly competitive labour market seems rather unrealistic. With the richest $5 \%$ of the population holding around $70 \%$ of the wealth and employing a mass of propertyless agents (around 50-70\% of the population), and the issues of power and class solidarity that this polarised wealth distribution raises, it seems natural to analyse a more complex model for the determination of the key distributive variables. 
In the next section, we extend our analysis to explore the interplay of accumulation and technical change in more general economies with full employment, generalised Nash bargaining, and population growth.

\section{Endogenous technical change with collec- tive bargaining on wages and profits}

In this section, we analyse exploitation and class in capitalist economies with technical change and population growth, by focusing on dynamic paths characterised by the full employment of labour. The key point to note is that whenever the economy is at the knife-edge, with $L_{t} A_{t}^{-1} \omega_{t-1}=l_{t}$, the equilibrium distribution is generally indeterminate. This allows us to explore the effects of bargaining on exploitation and class.

\subsection{The bargaining model}

Consider any period $t$ such that $L_{t} A_{t}^{-1} \omega_{t-1}=l_{t}$. For given technology and population, we assume that income distribution is determined by collective bargaining at the beginning of $t$, before the production process starts. To be specific, given $p_{t}=1$, the bargaining game $S\left(A_{t}, L_{t}\right)$ is defined as ${ }^{17}$

$$
S\left(A_{t}, L_{t}\right) \equiv \cup_{\widehat{w}_{t} \in\left[b, \frac{1}{v_{t}}\right]}\left\{\left(s^{\nu}\right)_{\nu \in \mathcal{N}_{t}} \mid s^{\nu} \leqq V_{t}^{\nu}\left(\omega_{t-1}^{\nu} ;\left(1, \widehat{w}_{t}\right)\right) \quad\left(\forall \nu \in \mathcal{N}_{t}\right)\right\},
$$

where $V_{t}^{\nu}\left(\omega_{t-1}^{\nu} ;\left(1, \widehat{w}_{t}\right)\right)=\left(1+\pi_{t}\right) \omega_{t-1}^{\nu}+\left(\widehat{w}_{t}-b\right) l_{t}^{\nu}$, all $\nu \in \mathcal{N}_{t}$, and so the set of Pareto-optimal payoffs is completely identified by varying $\widehat{w}_{t}$. Let $\sigma_{t} \equiv\left(\sigma_{t}^{\nu}\right)_{\nu \in \mathcal{N}_{t}} \in \mathbb{R}_{+}^{N_{t}}$ with $\sum_{\nu \in \mathcal{N}_{t}} \sigma_{t}^{\nu}=1$ denote a profile of weights capturing the agents' bargaining power. The Nash bargaining solution $N^{\left(\sigma_{t}\right)}\left(S\left(A_{t}, L_{t}\right)\right)$ is:

$$
N^{\left(\sigma_{t}\right)}\left(S\left(A_{t}, L_{t}\right)\right)=\arg \max _{\widehat{w}_{t} \in\left[b, \frac{1}{v_{t}}\right]} \prod_{\nu \in \mathcal{N}_{t}}\left[V_{t}^{\nu}\left(\omega_{t-1}^{\nu} ;\left(1, \widehat{w}_{t}\right)\right)\right]^{\sigma_{t}^{\nu}} .
$$

There are many conceivable ways of capturing agents' bargaining power. Different specifications yield different dynamics of income distributions and exploitative relations. In what follows, we consider a large set of possibilities by specifying the bargaining power of each agent $\nu \in \mathcal{N}_{t}$ as follows:

$$
\sigma_{t}^{\nu} \equiv(1-\epsilon) \frac{\omega_{t-1}^{\nu}}{\omega_{t-1}}+\epsilon \frac{N_{t}^{\nu}}{N_{t}}, \text { some } \epsilon \in[0,1]
$$

\footnotetext{
${ }^{17}$ In this section, we focus on the bargaining procedure and assume again a constant $b$.
} 
where $N_{t}^{\nu} \geq 1$ is the number of agents who possess the same wealth as $\nu$. Formally, $N_{t}^{\nu} \equiv \#\left\{\nu^{\prime} \in \mathcal{N}_{t} \mid \omega_{t-1}^{\nu^{\prime}}=\omega_{t-1}^{\nu}\right\}$.

Equation (7) incorporates some important insights of Marxian theory. For it states that the bargaining power of each agent $\nu \in \mathcal{N}_{t}$ depends partly on the ownership of means of production (more precisely, on their share of aggregate capital) and partly on the number of agents who share the same objective condition as $\nu$ (in terms of capital ownership). Thus, bargaining power derives either from economic resources, or from the ability of agents in a similar condition to act collectively.

Equation (7) is very general. The variable $\epsilon$ allows us to capture the polar cases of economies with weak solidarity and low levels of organisation $(\epsilon \approx 0)$, and economies in which the power of capital and economic resources is mitigated by collective action $(\epsilon \approx 1)$, as well as any intermediate scenarios. If $\epsilon=0$, then the bargaining power of each agent is proportional to her share of aggregate capital: capitalists have all the bargaining power while propertyless agents have no influence at all on the determination of wages and profits. In contrast, if $\epsilon=1$, then - given a very skewed distribution of wealth - the richer segments of the population have virtually no bargaining power: propertyless agents constitute the majority of population and play the main role in determining bargaining outcomes.

Let $\widehat{w}_{t}^{N^{\left(\sigma_{t}\right)}} \in N^{\left(\sigma_{t}\right)}\left(S\left(A_{t}, L_{t}\right)\right)$ : in period $t$, every agent $\nu \in \mathcal{N}_{t}$ solves $M P_{t}^{\nu}$ given the price vector $\left(1, \widehat{w}_{t}^{N^{\left(\sigma_{t}\right)}}\right)$, and given $\left(\left(A_{t}, L_{t}\right), b\right)$. Because $L_{t} A_{t}^{-1} \omega_{t-1}=l_{t}$, it is immediate to show that conditions (a)-(d) of Definition 1 are satisfied.

At the end of the period, both technological knowledge and population are updated. Concerning the former, let $\pi_{t}^{N^{\left(\sigma_{t}\right)}}=\frac{1-A_{t}-\widehat{w}_{t}^{N^{\left(\sigma_{t}\right)}} L_{t}}{A_{t}}$ be the profit rate resulting from the bargaining process. As in section 7 , if $\pi_{t}^{N^{\left(\sigma_{t}\right)}}<\pi^{*}$, then capitalists redouble their efforts to find new profitable techniques. We assume that in this case a new technique is generated with probability $\lambda \leqq 1$, such that $A_{t+1}=g A_{t}$ and $L_{t+1}=g L_{t}$, for some $g<1$. The new technique is obviously cost reducing and therefore is universally adopted in period $t+1$.

Concerning population, we assume that economic growth drives population growth - for example, by determining population flows in or out of the economy, or as part of a general Malthusian mechanism. Formally, in each $t$, if the economy is at a knife-edge equilibrium, then population in period $t+1, l_{t+1}$, will be

$$
l_{t+1}=\left[\left(1-b L_{t}\right) A_{t}^{-1}\right] l_{t},
$$

where $\left[\left(1-b L_{t}\right) A_{t}^{-1}\right]$ corresponds to the growth rate of capital at $t$ and 
can be interpreted, in a classical-Marxian fashion, as the natural rate of population growth. For it is reasonable to assume that in a situation of full utilisation of capital and labour, the growth rate of population is adjusted to be equal to the rate of capital accumulation. ${ }^{18}$

The combined effect of our assumptions on technical change and population growth is that if the economy is at a full employment equilibrium in period $t$, there will be neither excess capital nor excess labour in $t+1$. To be sure, both assumptions can be deemed somewhat artificial: they should be interpreted as a schematic representation (due to the linear structure of the model and the peculiarity of the knife-edge condition) of a self-correcting mechanism, or as part of a balanced growth path.

\subsection{Simulation Results}

The simulation begins with the same $N_{0}=100$ and the same distribution of wealth as in all previous models, and with initial values of $A_{0}=0.9$, $L_{0}=1$, and $b=0.08$. For the technical change routine the threshold profit rate is $\pi^{*}=0.03$ and the likelihood of discovering a new technique when appropriate is $\lambda=1$. When technical change takes place, the value of $g$ is randomly drawn from a uniform distribution from 0.9 to 0.96 . As concerns the bargaining parameter $\epsilon$ in equation (7), below we consider three different scenarios, namely the two polar cases with capitalist dominance $(\epsilon=0)$ and class solidarity $(\epsilon=1)$, and the intermediate case with $\epsilon \in(0,1)$.

The simulation occurs in the following order: (1) determine $\left(\sigma_{t}^{\nu}\right)_{\nu \in \mathcal{N}_{t}} ;(2)$ determine $\widehat{w}_{t}^{N\left(\sigma_{t}^{\nu}\right)}$ and $\pi_{t}$ through Nash bargaining; (3) agents solve $M P_{t}^{\nu}$; (4) population $l_{t+1}$ is determined to balance the knife-edge condition; ${ }^{19}$ (5) $A_{t+1}$ and $L_{t+1}$ are updated if appropriate; (6) steps (2)-(5) are repeated for $T$.

\footnotetext{
${ }^{18}$ In a more general setting allowing for unemployment of labour or capital, one could assume that if the economy is capital constrained at $t$, then $l_{t+1}<\left[\left(1-b L_{t}\right) A_{t}^{-1}\right] l_{t}$; while if the economy is labour constrained then $l_{t+1}>\left[\left(1-b L_{t}\right) A_{t}^{-1}\right] l_{t}$. This is because if the economy is capital constrained and labour is abundant then the wage rate is at the subsistence level and, in the Malthusian law of population, labour supply grows slowly, while if the economy is labour constrained and capital is abundant, profits are zero and the real wage is very high, inducing a high growth rate of the population.

${ }^{19} \mathrm{In}$ order to ensure that $l_{t}=L_{t} A_{t}^{-1} \omega_{t-1}$ holds for all $t, N_{t}$ is determined by rounding $L_{t} A_{t}^{-1} \omega_{t-1}$ up to the nearest integer. Therefore $\omega_{t-1}$ is also adjusted upward to maintain the knife-edge. Any additional capital is added to the endowment of the wealthiest agent in order to avoid random changes in the behaviour of the simulation. Any new agents enter the simulation with $\omega_{t-1}^{\nu}=0$. This is reasonable as a first approximation, or if one interprets population growth mainly as the product of migration flows.
} 


\subsubsection{Capitalist dominance}

Consider first the case where economic resources, and specifically ownership of the means of production, are the key determinant of bargaining power and $\epsilon=0$ in equation (7). Figure 8 reports the summary results of the aggregate activity levels $\left(y_{t}, x_{t}, z_{t}, \delta_{t}\right)$, net output $\left(1-A_{t}\right) y_{t}$, net output per capita $\left(1-A_{t}\right) y_{t} / N_{t}$, wealth $W_{t-1}$, the growth rate of capital $g_{t}$, and the distributive variables $\left(\widehat{w}_{t}^{N\left(\sigma_{t}^{\nu}\right)}, \pi_{t}\right)$. Two features immediately stand out. First, the combination of population growth and wage bargaining leads to rapid accumulation. Even over a relatively short length of time $T=50$, population grows from 100 agents to 32,485. Second, the bargaining power of capitalists is such as to guarantee that the profit rate remains positive and at a rather high level, while $\widehat{w}_{t}^{N\left(\sigma_{t}^{\nu}\right)}=b$ for all $t$, even though the economy never becomes capital constrained. Figure 9 displays $A_{t}, L_{t}$, and $v_{t}$.

Figures 10-11(b) show the exploitation and class status of agents. ${ }^{20}$ They show a distinct, remarkable pattern of increasing polarisation in the economy. As time goes by, an ever increasing proportion of the agents are exploited by an ever decreasing minority of exploiters. The fraction of agents in the lower classes constantly rises while that of agents in the upper classes falls.

One interesting result from this simulation is that the Gini coefficient of $e_{t}^{\nu}$ decreases over time. This is not because exploitation is disappearing from the economy, it is an artifact of the way in which Gini coefficients are constructed. The ever-increasing number of agents with $\omega_{t-1}^{\nu}=0$ leads to the vast majority of agents having identical, high values of $e_{t}^{\nu}$, which leads the Gini of $e_{t}^{\nu}$ to decrease as shown in Figure 11(a). Given the clear pattern of increasing polarisation emerging from Figure 10, this suggests that perhaps the Gini coefficient is not the best index of the aggregate degree of exploitation, and it raises the issue of the appropriate aggregate measure of exploitative relations. ${ }^{21}$

\footnotetext{
${ }^{20}$ Unlike in previous models, given the rapidly changing population over time, in this section the charts reporting class and exploitation status show the percentage of the population at all $t$ belonging to a given class or exploitation classification.

${ }^{21}$ The polarisation of the economy also shows up if one considers the distributions of wealth and income (taking income as the gross revenue of agents as determined by the left-hand side of constraint (1) in $M P_{t}^{\nu}$ ), whose Gini coefficients monotonically increase over time, tending to a value above 0.9 in the long run. See section A.4 of the Appendix.
} 
Figure 8: Summary results - Bargaining model with $\epsilon=0$
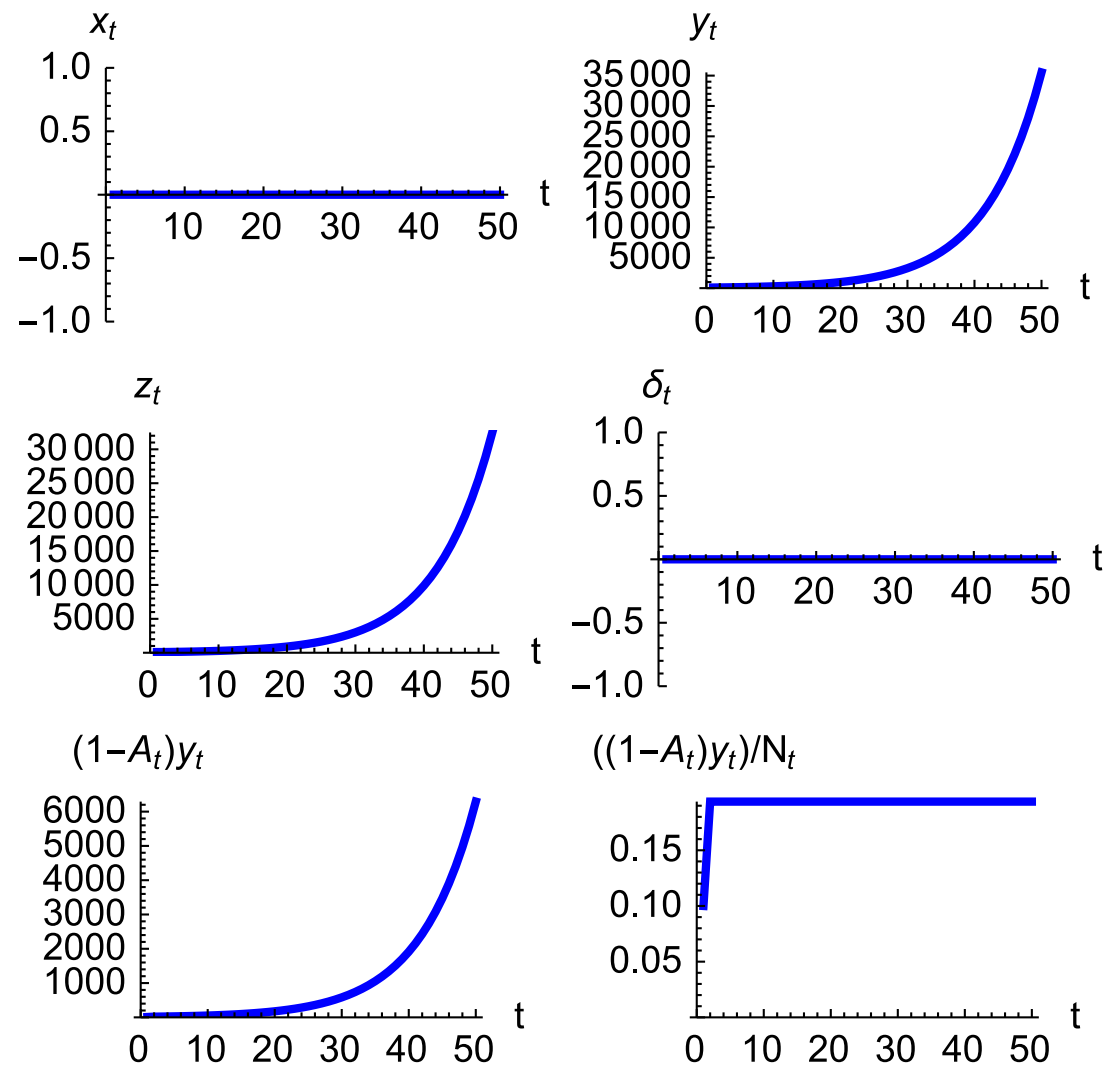

$\left(\left(1-A_{t}\right) y_{t}\right) / \mathrm{N}_{t}$

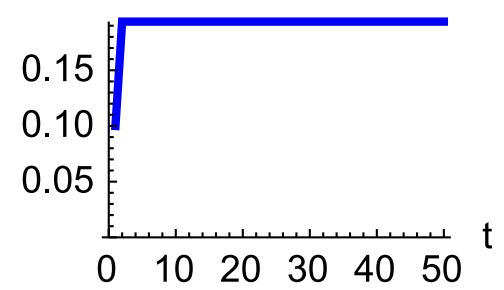

$W_{t-1}=p_{t-1} \omega_{t-1}$
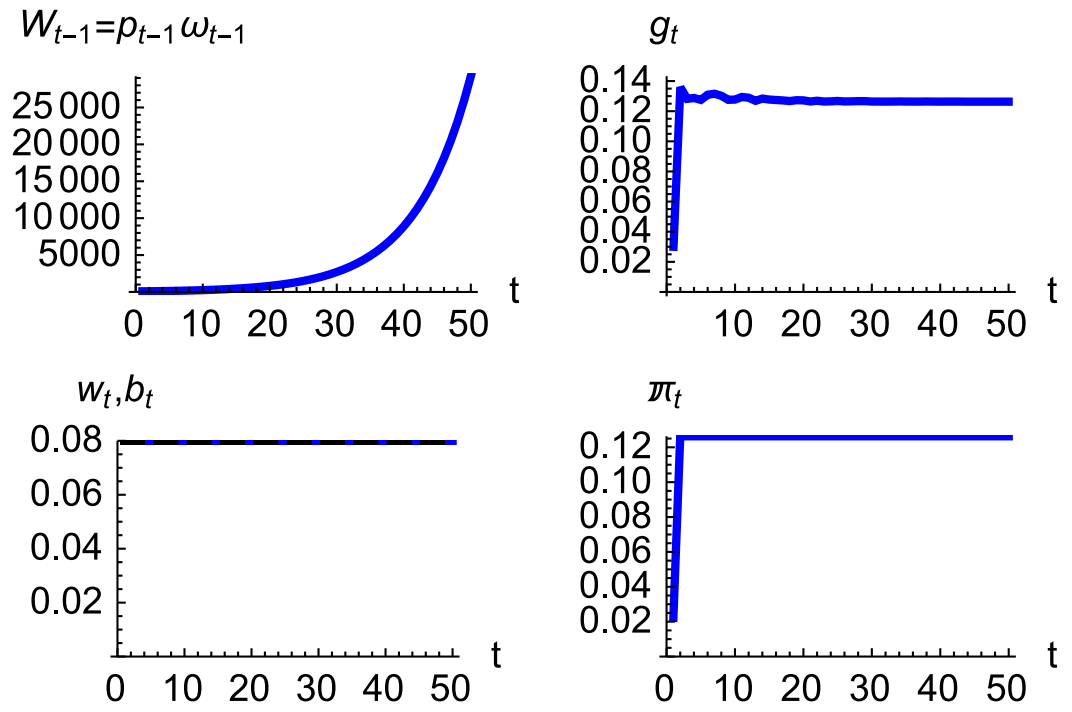
Figure 9: Technology and labour values - Bargaining model with $\epsilon=0$
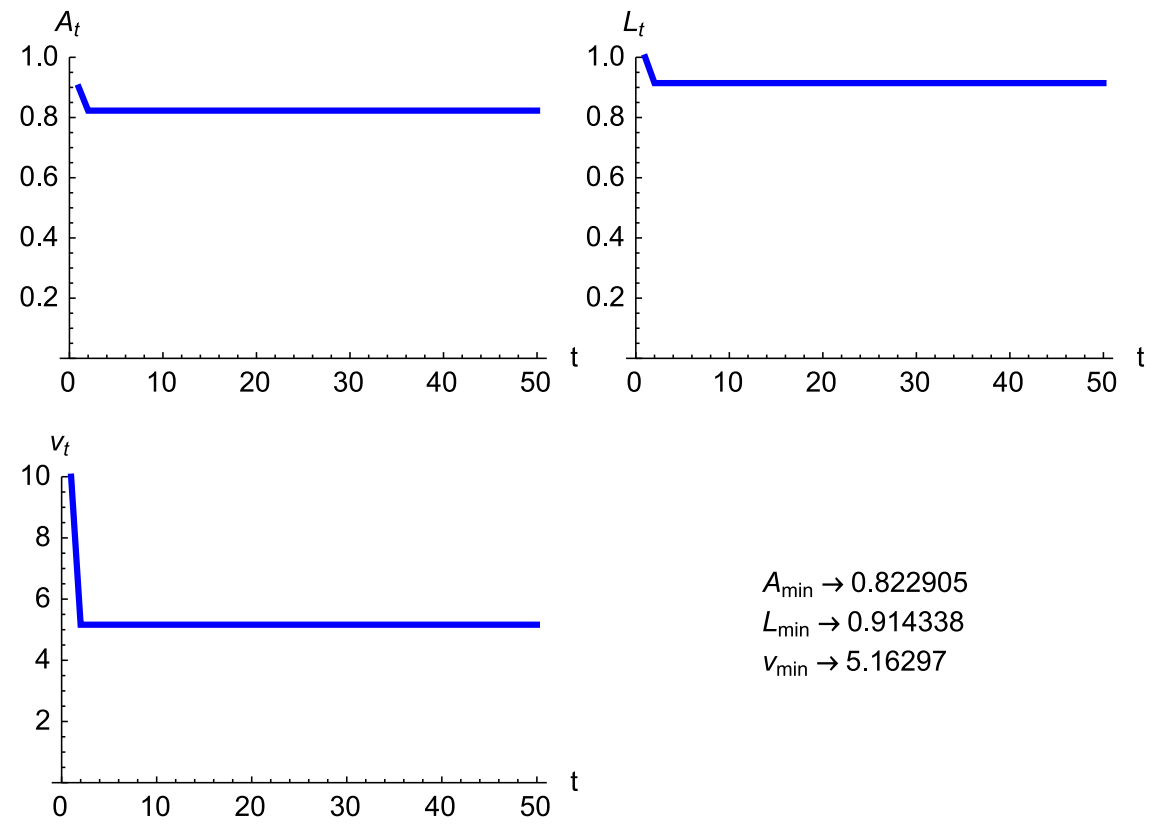

\subsubsection{Class solidarity}

Consider next the case where bargaining power is determined solely by class solidarity and $\epsilon=1$ in equation (7). The results are presented in Figures 12 and 13. The economy displays a rather fast pace of accumulation as in the model with capitalist dominance. Given the numerical preponderance of the agents with $\omega_{t-1}^{\nu}=0$, however, the power of class solidarity in wage bargaining favours propertyless agents who set a value of $\widehat{w}_{t}^{N\left(\sigma_{t}^{\nu}\right)}$ leading to $\pi_{t}=0$ for all $t \geq 1$. In other words, working class solidarity eliminates exploitation from the very beginning and the benefits of accumulation (and productivity increases) are distributed in the form of increased wages. The figures reporting exploitation status, class status, and the CECP are not shown as they do not convey much information. The Gini of the exploitation intensity index is obviously equal to zero throughout the simulation. ${ }^{22}$

\footnotetext{
${ }^{22}$ The Gini coefficients of wealth and income display an interesting behaviour over time and provide a rather different picture of the economy, compared to the Gini coefficient of $\left(e_{t}^{\nu}\right)_{\nu \in \mathcal{N}_{t}}$. The Gini coefficient of wealth initially decreases, reflecting the reduction in inequalities due to the zero profit rate and the accumulation of wealth by all agents (since $\widehat{w}_{t}>b$ all $\left.t\right)$. This trend is then reversed as growth accelerates and the number of new, propertyless agents added to economy in each $t$ grows closer to (and eventually surpasses) the number of existing agents with a positive (and possibly quite large) amount of wealth
} 
Figure 10: Class and exploitation status - Bargaining model with $\epsilon=0$

(a) Exploitation status

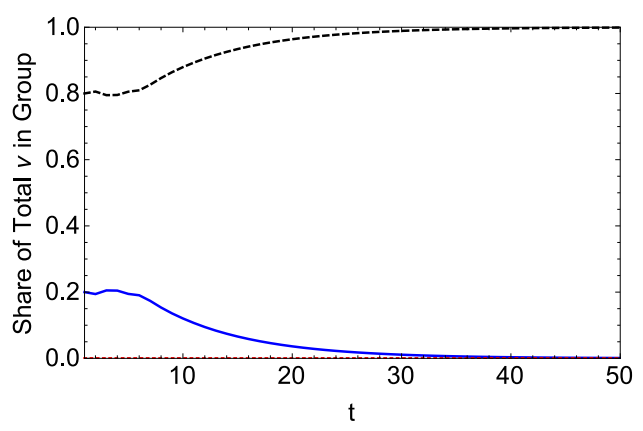

(b) Class status

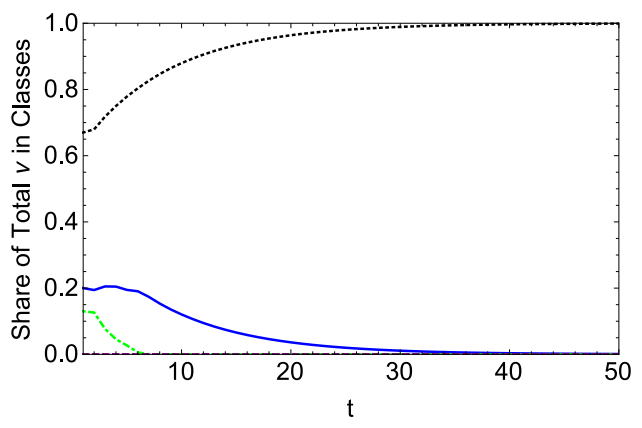

(c) CECP

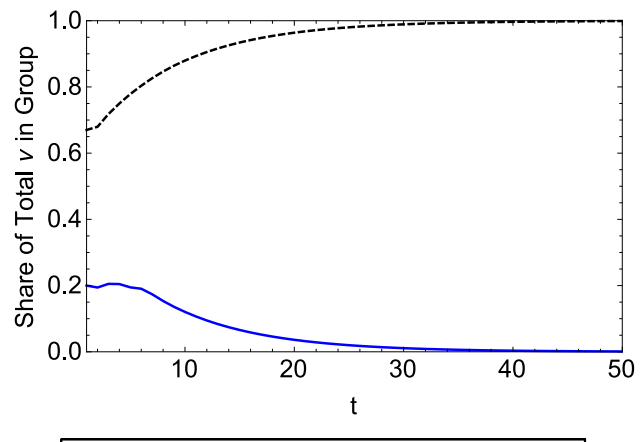

$-C_{t}^{1} \wedge$ Exploiter

\subsubsection{Mixed scenarios}

In this section, we consider economies in which $\epsilon \in(0,1)$ and the bargaining power of agents derives both from the ownership of the means of production and from class solidarity and collective action. The fundamental, and rather striking result of the computational analysis is that class solidarity plays a dominant role in the bargaining process and tends to drive the dynamics of the economy. For instance, for any $\epsilon \geq 0.5$, the economy behaves in a manner

accumulated over time. In contrast, the Gini coefficient of income (defined as the agents' gross revenue) exhibits a persistent downward trend during the simulation. This is due to the fact that by construction the wage income of all agents is the same and the zero profit rate implies that asset inequalities only partly translate into income inequalities. (See section A.4 of the Appendix.) 
Figure 11: Information on $e_{t}^{\nu}$ - Bargaining model with $\epsilon=0$

(a) Gini coefficient of $e_{t}^{\nu}$

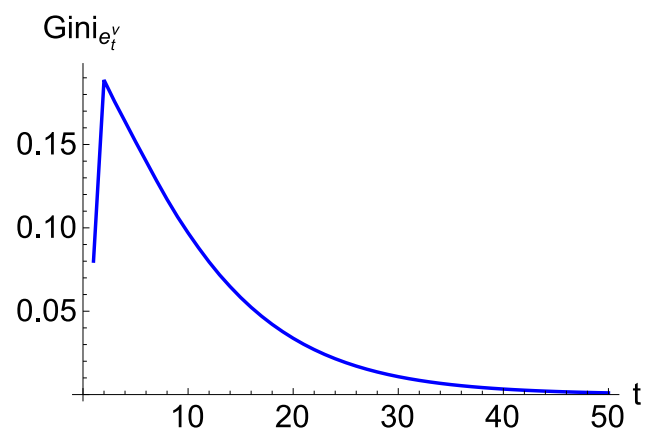

(b) Distribution of $e_{t}^{\nu}$ for select $t$ (relative frequency)

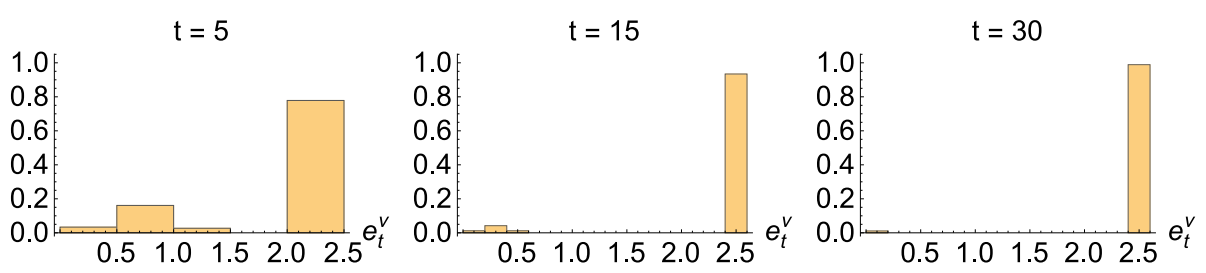

qualitatively similar to the model with $\epsilon=1$ : the bargaining process leads to a value of $\widehat{w}_{t}^{N\left(\sigma_{t}^{\nu}\right)}$ such that $\pi_{t}=0$ for all $t$.

Only very small values of $\epsilon$ - in the range of 0.001 to 0.005 - allow for behaviour along the knife-edge at which $\pi_{t}>0$ and $\widehat{w}_{t}^{N\left(\sigma_{t}^{\nu}\right)}>b$, but only for a small number of periods. For $\epsilon \in[0.001,0.005]$, the economy displays a very interesting cyclical behaviour: the bargaining power of propertyless agents drives profits initially to zero but, once technical change occurs, the profit rate starts to increase, and accumulation with it. However, as population also grows, the number of new (propertyless) agents added in every $t$ is large and their class solidarity begins to outweigh the power of relatively wealthy agents. This leads the profit rate to decrease and even though - when $\pi_{t}$ falls below $\pi^{*}$ - other technical innovations are introduced, they only allow for a short-lived recovery of profitability. The economy then enters a phase during which profits and exploitation disappear for any remaining $t$.

Below, we show the results of the simulation for $\epsilon=0.002$. Figure 14 reports the summary results of the aggregate activity levels $\left(y_{t}, x_{t}, z_{t}, \delta_{t}\right)$, net output $\left(1-A_{t}\right) y_{t}$, net output per capita $\left(1-A_{t}\right) y_{t} / N_{t}$, wealth $W_{t-1}$, 
Figure 12: Summary results - Bargaining model with $\epsilon=1$
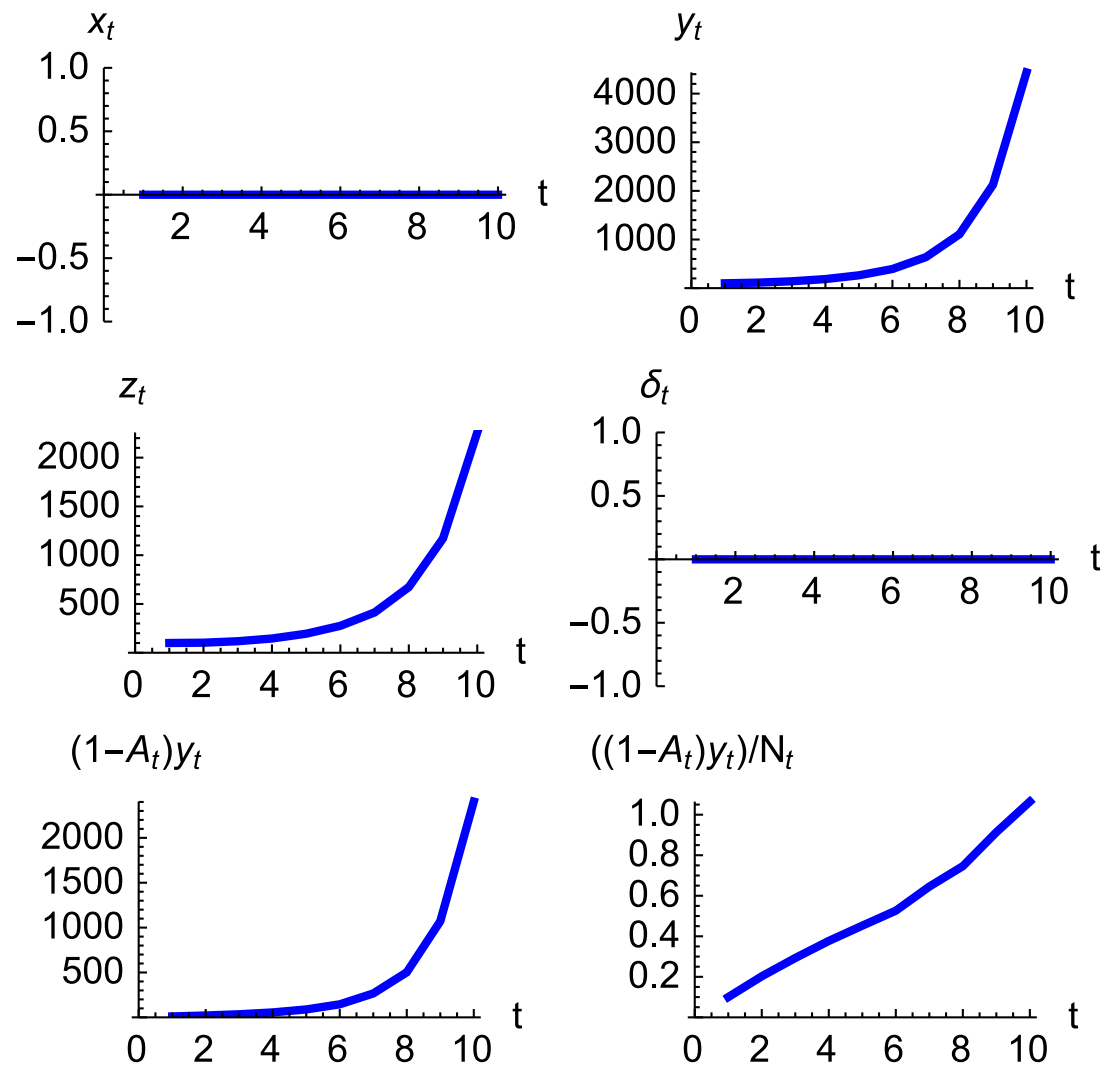

$\left(\left(1-A_{t}\right) y_{t}\right) / \mathrm{N}_{t}$

$W_{t-1}=p_{t-1} \omega_{t-1}$
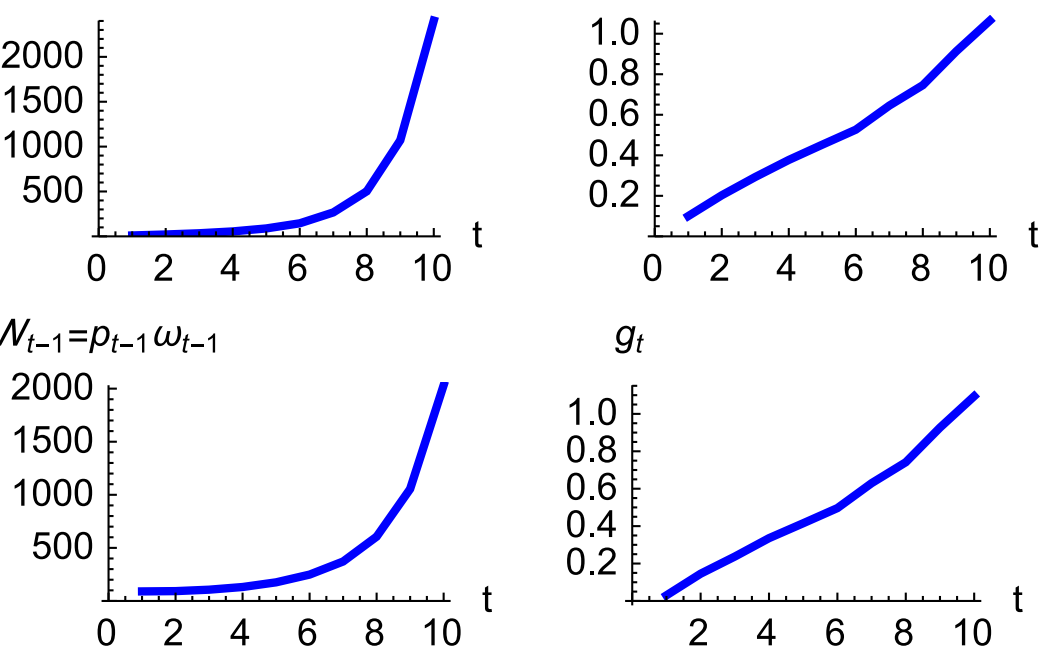

$$
w_{t}, b_{t}
$$

1.0
0.8

0.6

0.4

0.2
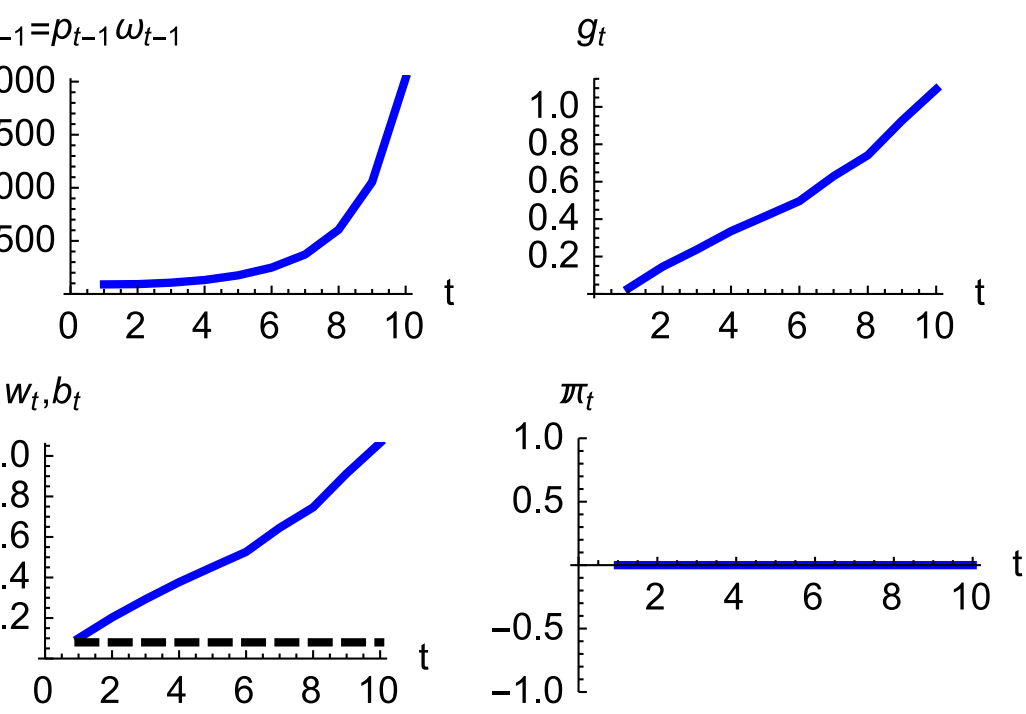
Figure 13: Technology and labour values - Bargaining model with $\epsilon=1$
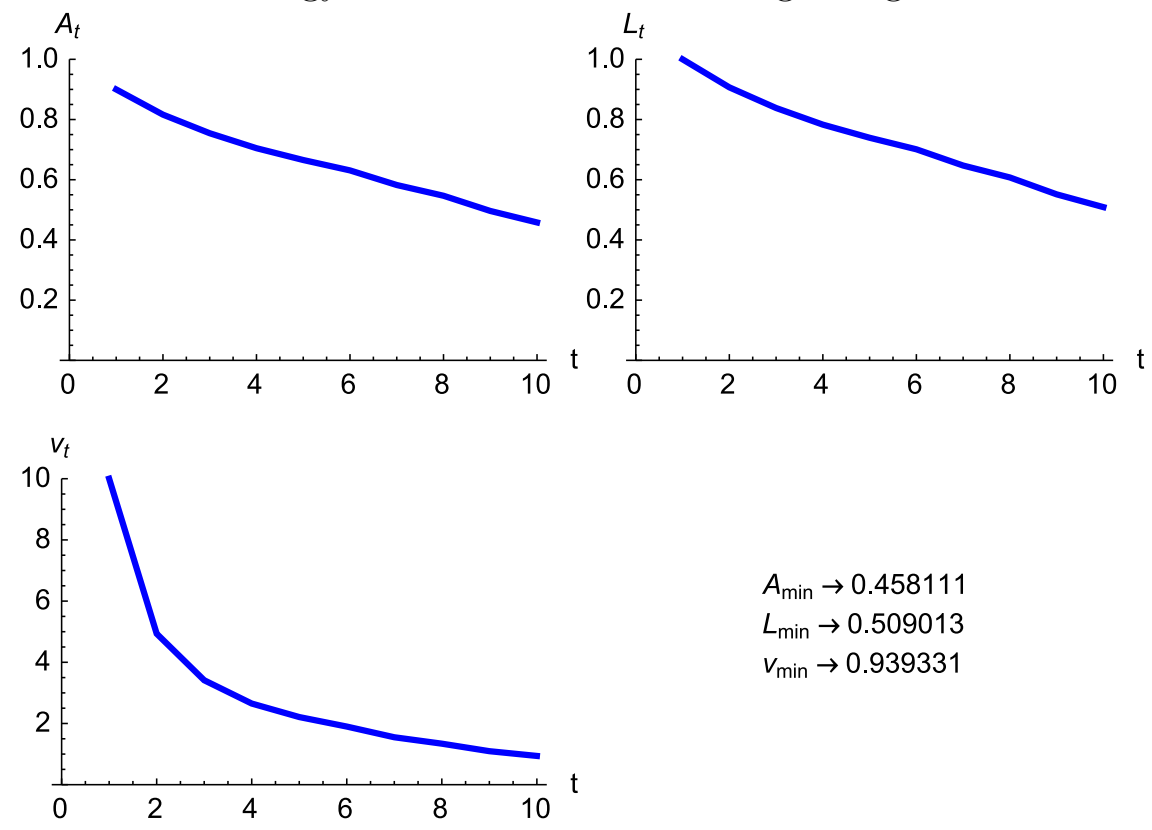

the growth rate of capital $g_{t}$, and the distributive variables $\left(\widehat{w}_{t}^{N\left(\sigma_{t}^{\nu}\right)}, \pi_{t}\right)$. As in the previous cases, the combination of population growth and wage bargaining leads to rapid accumulation. Even over a short length of time $T=20$, the population grows from 100 agents to 8,556 . The bottom right panel captures the cycle in the profit rate described above, where $\pi_{t}=0$ for all $t \geq 15$. Figure 15 displays $A_{t}, L_{t}$, and $v_{t}{ }^{23}$

Figures 16(a)-16(c) show the exploitation and class status of agents. Figure 16(a) captures the lack of exploitation at the start of the simulation, its reemergence for a period of time, and its eventual disappearance. Figure 16(b) reports the dynamics of classes, which is richer than in previous models. Observe that, whenever the real wage is above the subsistence level, all agents in $C_{t}^{4}$ accumulate and join $C_{t+1}^{2}$, and are fully replaced in $C_{t+1}^{4}$ by the newly arrived agents with $\omega_{t}^{\nu}=0$. Hence, the economy shows some upward mobility. As accumulation progresses, wage bargaining mediates the movement of some agents between $C_{t}^{1}$ and $C_{t}^{2}$, but the continued expansion of $C_{t}^{4}$ and the faster pace of accumulation induce a downward trend in the size of $C_{t}^{2}$ and an upward trend in that of $C_{t}^{1}$. The continual growth of $C_{t}^{4}$ (despite upward social mobility) reflects the arrival of an increasingly larger number of propertyless agents. Figure 16(c) captures the short period of

\footnotetext{
${ }^{23}$ The condition $v_{t} b_{t}<1$ holds throughout the simulation.
} 
Figure 14: Summary results - Bargaining model with $\epsilon=0.002$
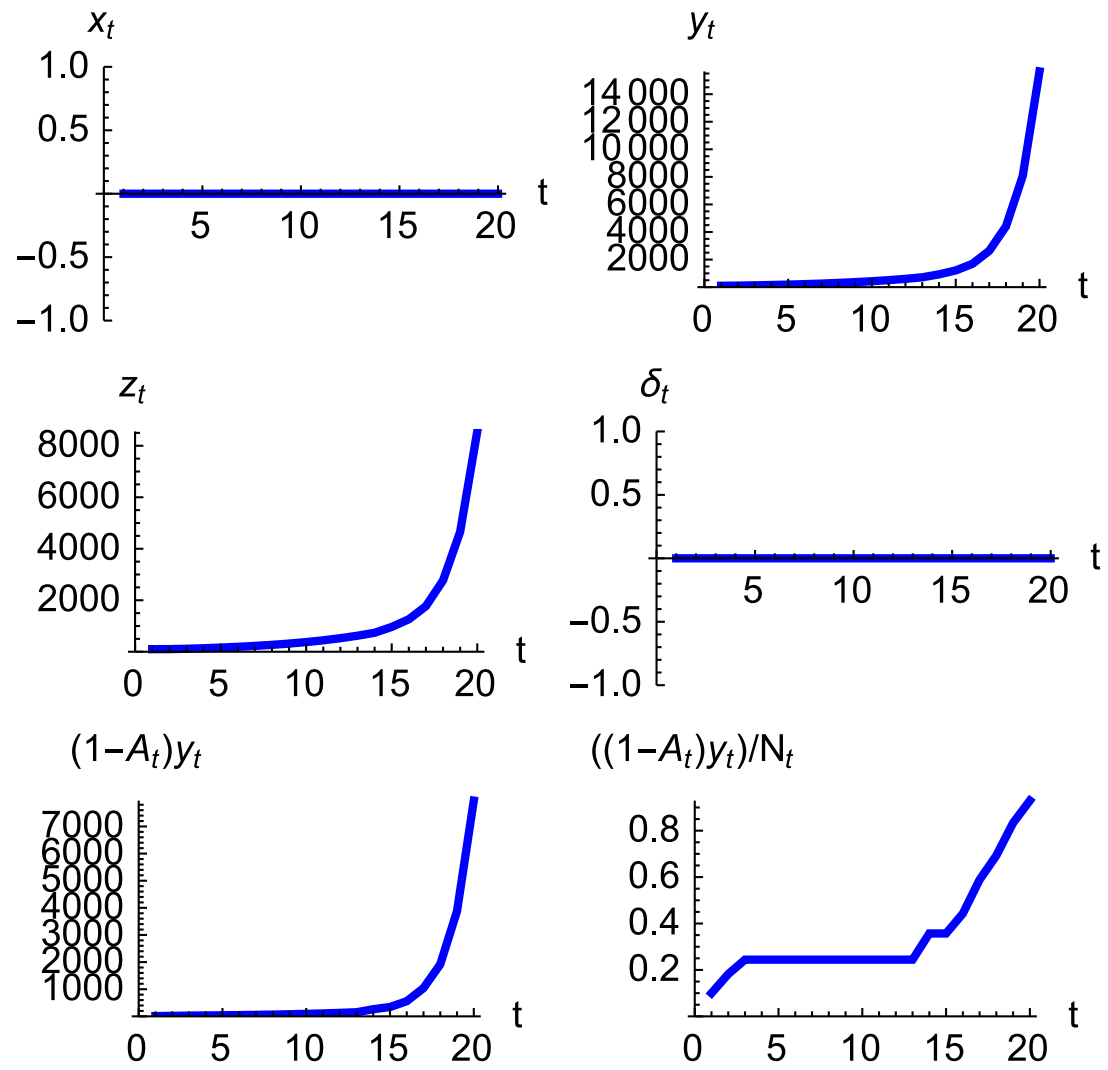

$\left(\left(1-A_{t}\right) y_{t}\right) / \mathrm{N}_{t}$

$W_{t-1}=p_{t-1} \omega_{t-1}$
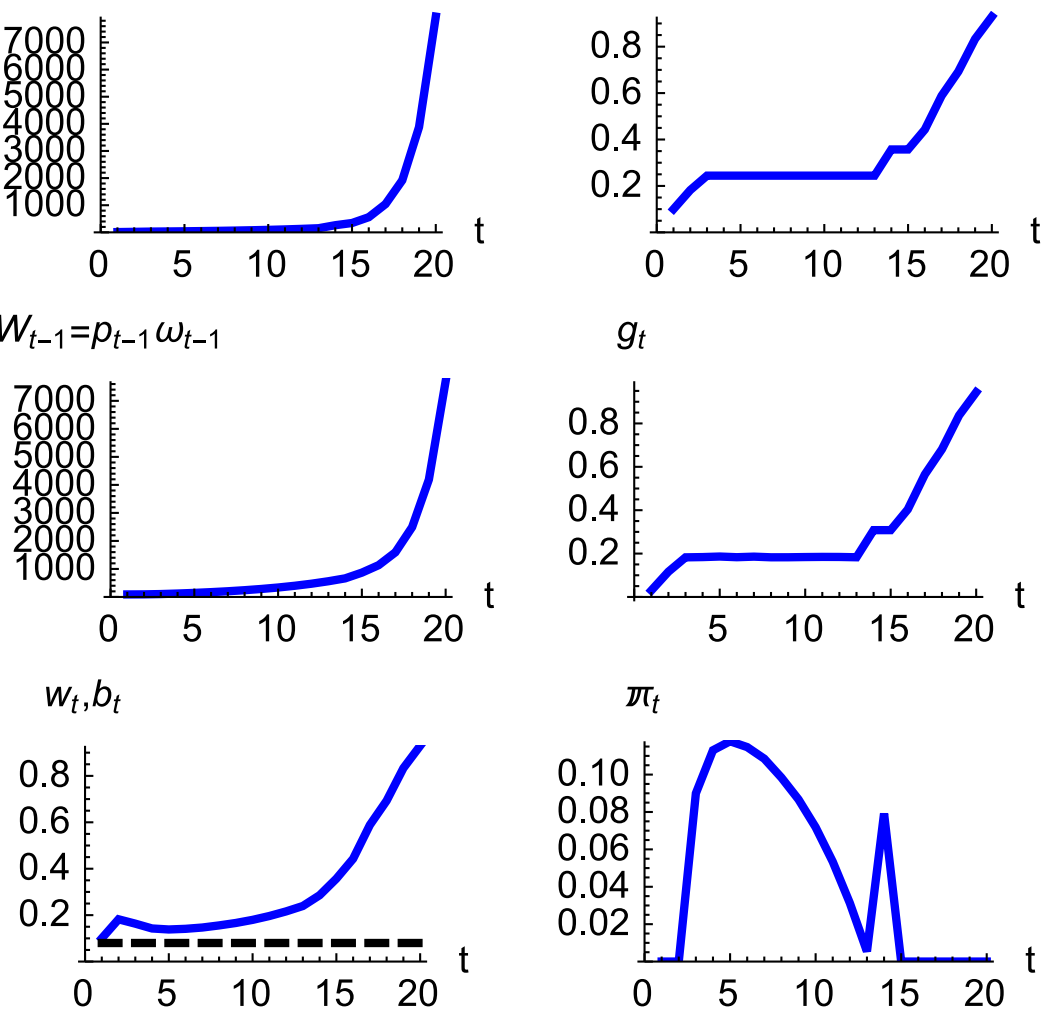
Figure 15: Technology and labour values - Bargaining model with $\epsilon=0.002$
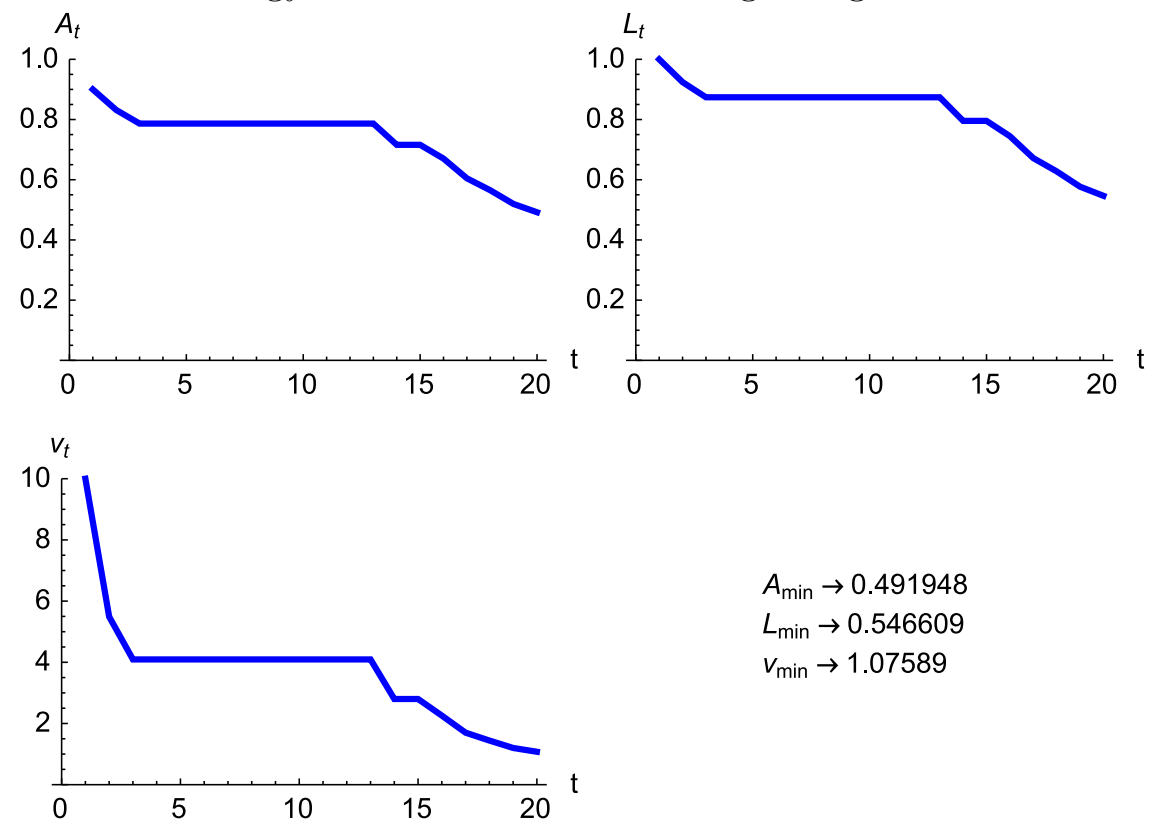

$$
\begin{aligned}
& A_{\min } \rightarrow 0.491948 \\
& L_{\min } \rightarrow 0.546609 \\
& v_{\min } \rightarrow 1.07589
\end{aligned}
$$

time for which capitalists are able to convert their ownership of the means of production into economic advantage and the CECP applies, after which the correspondence between class and exploitation status breaks down.

Figures 17(a) shows the Gini coefficient for $e_{t}^{\nu}$, which mirrors the cycle in the profit rate with exploitation intensity eventually converging to one for all agents once $\pi_{t}=0$. Snapshots of the distribution of $e_{t}^{\nu}$ for select $t$ are provided in Figure 17(b) to show the progression of exploitation intensity to uniformity as accumulation progresses. ${ }^{24}$

In closing this section, some interesting features of the economies with bargaining are worth noting. A comparison of the dynamic paths of the three models suggests that the class solidarity regime is best and the capitalist dominance regime is worst in terms of several indicators, including growth rates, capital accumulation, labour productivity, and per capita consumption. ${ }^{25}$ Of course, this may depend on the exact specification of our

\footnotetext{
${ }^{24}$ The interaction between varying distributional patterns and the changing composition of the population over time leads to patterns of the Gini coefficients for wealth and income (defined as gross revenue) that are on the whole qualitatively similar to those of the model with class solidarity. (See section A.4 of the Appendix.) The basic intuition is the same as explained in footnote 22 .

${ }^{25}$ Even population growth is lowest in the capitalist dominance regime and highest in
} 
Figure 16: Class and exploitation status - Bargaining model with $\epsilon=0.002$

(a) Exploitation status
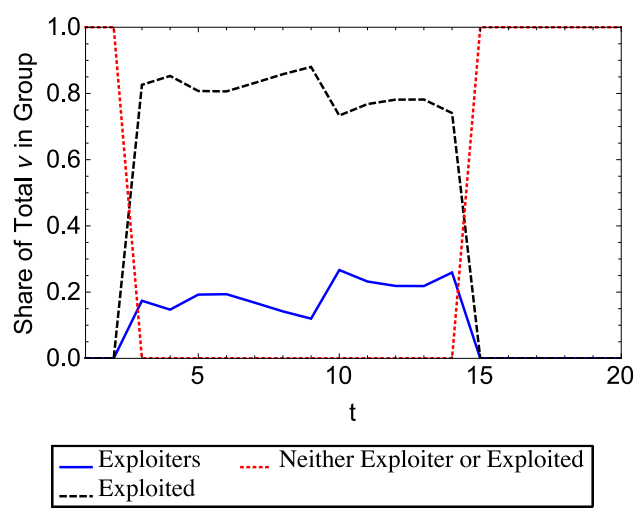

(b) Class status

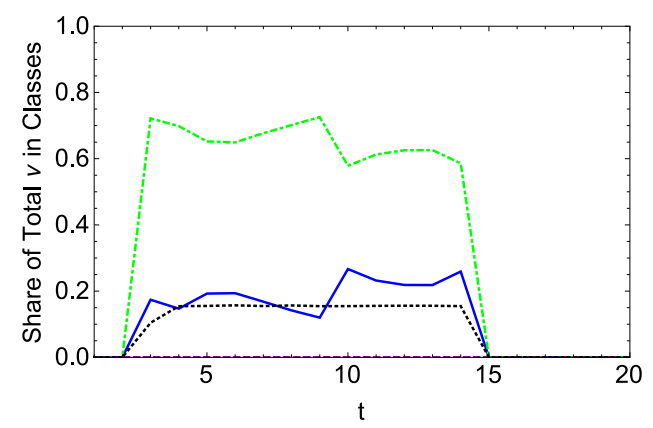

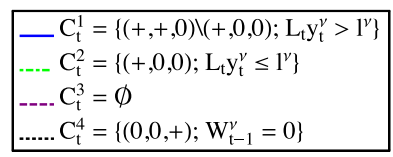

(c) CECP

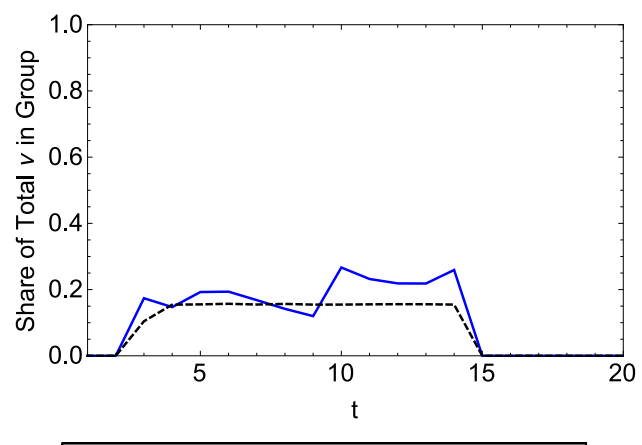

$-C_{t}^{1} \wedge$ Exploiter

model, including the assumptions guaranteeing that the economy remains along the knife-edge path. Yet, this insight is fundamentally consistent with the fact that capitalist economies with strong concentration of power in the hands of capitalists tend to settle on dynamic paths with high profit rates and low growth rates (at least relative to their potential), as forcefully shown by Piketty [12]. Indeed, in terms of our model, the trajectory of advanced capitalist economies in the last forty years (the neo-liberal era) might be explained as the product of a shift from a kind of mixed regime with a strong working class to a capitalist dominance regime.

the class solidarity regime, thus suggesting that the latter would be the most flourishing society. 
Figure 17: Information on $e_{t}^{\nu}$ - Bargaining model with $\epsilon=0.002$

(a) Gini coefficient of $e_{t}^{\nu}$

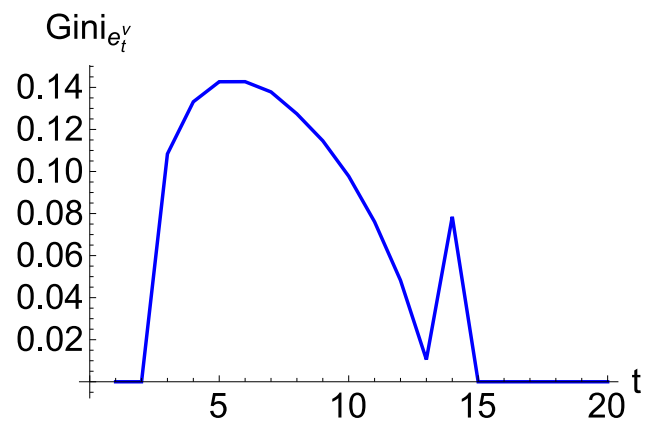

(b) Distribution of $e_{t}^{\nu}$ for select $t$ (relative frequency)

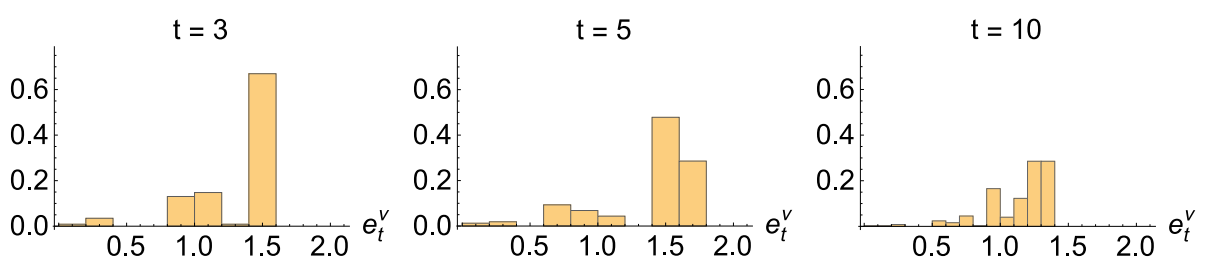

\section{Robustness}

We have analysed many variations of our models in order to assess the robustness of our results. In this section, we briefly summarise the main points. ${ }^{26}$

First, we have considered alternative specifications of the initial values of the key parameters of all models. Different values of the initial population, $\mathcal{N}_{0}$, technology, $\left(A_{0}, L_{0}\right)$, consumption bundle, $b_{0}$, and aggregate capital, $\omega_{0}$, make hardly any difference to our conclusions. ${ }^{27}$ Different distributions of the initial aggregate capital, $\omega_{0}$, also have no impact on the results, provided capital is unequally distributed and there are some propertyless agents.

Changes in these parameters may affect the quantitative features of the economies (e.g., the size of the various classes, the period in which the economy becomes labour constrained, and so on) but the qualitative patterns of

\footnotetext{
${ }^{26}$ Results are available from the authors upon request.

${ }^{27}$ Subject, obviously, to the economy remaining capital constrained or on the knife edge, depending on the model.
} 
class and exploitation status, as well as the basic summary results, remain essentially unchanged. It is worth noting, however, that different values of the initial parameters yield different distributions of $e_{t}^{\nu}$. For example, a simulation of the basic economy with the same population and asset distribution as in section 3 , and $(A=0.5, L=0.25, b=0.5)$ yields a distribution of $e_{t}^{\nu}$ in the range $[0.03306,4]$ with a Gini coefficient of 0.26568 - quite close to the Gini coefficient of income in actual capitalist economies.

Second, we have analysed the robustness of the main results on class structure and on the relation between exploitation and class status by adopting an alternative definition of classes. Corollary 1 proves that class status is determined for each agent by the difference between actual labour demand and potential labour supply - i.e., the agent's labour endowment. Yet one may argue that class status should be determined based on agents' actual position in the labour market, and so on their actual labour supply. All of our main insights continue to hold if this alternative approach is adopted. ${ }^{28}$

Third, the results of the model with technical change and growth in consumption in section 7 are also strongly robust to alternative specifications of the functions $f_{1}$ and $f_{2}$, including different values of the dampening parameter $\phi$ and of the reference profit rate $\pi^{*}$, as well as to different assumptions on the properties of new techniques. Among other tests, we have analysed an alternative specification of the model in which the new technique $\left(A^{\prime}, L^{\prime}\right)$ is chosen by first randomly selecting a change in $A_{t}$ from a uniform distribution between 0.03 and 0.05 . Then the new value of $L_{t}$ is determined by setting $v_{t} b_{t}=v_{1} b_{1}+h$, where $b_{t}$ is given by equation (6) and $h$ is a random real number in the interval $[-0.25,0.25] .{ }^{29}$ To ensure that $A_{t}<1$ for all $t$ there is a maximum value set at $A_{\max }=0.991$ : once $A_{\max }$ is reached, technical change continues as Harrod-neutral (constant $A_{\max }$ and decreasing $L_{t}$ when $\left.\pi_{t}<\pi^{*}\right)$ to ensure that $1>v_{t} b_{t}$. An interesting property of this model is that - unlike in section 7 - innovations do not automatically restore profitability. The results obtained are strikingly similar to those presented in section $7 .{ }^{30}$

We have also tested alternative assumptions on the timing of technical change by supposing that the decision to implement technical change is taken based on realised rather than anticipated profit rates. Formally, this implies assuming that, when $\pi_{t}<\pi^{*}$, innovations occur after production takes place. The main insights in section 7 remain valid with classes and exploitation behaving in a qualitatively similar way. Technical change does yield persistent

\footnotetext{
${ }^{28}$ See Section A.3 of the Appendix.

${ }^{29}$ These assumption ensure that the condition, $1>v_{t} b_{t}$, is never violated.

${ }^{30}$ See Section A.2 of the Appendix.
} 
exploitation and profits on the whole, but since it occurs ex post the profit rate is not necessarily strictly positive in every period.

Fourth, section 8 already reports the results for a large set of values of the bargaining parameter $\epsilon$. But the key insights are robust to changes in various other features of the model. For example, the main results remain essentially unchanged if we relax the assumption that the likelihood of discovering a new technique when appropriate is exactly equal to one, or if we allow technical change to take place at a faster pace by increasing the range of $g$. Perhaps more interestingly, in equation (7), instead of defining $N_{t}^{\nu}$ as the number of agents who possess exactly the same amount of wealth as $\nu$, one may adopt a broader categorisation and define $N_{t}^{\nu}$ as the number of agents with wealth within a certain interval, ${ }^{31}$ or who belong to the same class as $\nu .{ }^{32}$ These alternative specifications do not change the results significantly given the rather unequal wealth distribution, and given our specification of population growth. For in all of these scenarios the bargaining power of the poorest segments of the working class remains unchanged, and it increases over time as more propertyless agents appear in the economy.

\section{Conclusions}

This paper analyses the equilibrium dynamics of exploitation and class in general accumulation economies with population growth, technical change, and bargaining by adopting a novel computational approach. Two sets of results emerge from the analysis. First, in capitalist economies characterised by a drive to accumulate, labour-saving technical change plays a key role in guaranteeing the persistence of exploitation and class by making labour persistently abundant relative to capital. Nonetheless, and perhaps strikingly, in competitive economies characterised by full employment and bargaining over distribution, labour-saving technical change and population growth are not sufficient to generate persistent class and exploitative relations even if the economy never becomes labour constrained. The model forcefully highlights the importance of power, and specifically bargaining power, as one of the possible determinants of the persistence of classes and exploitation. It is only when economic resources, and specifically the ownership of the means of production, give a significant advantage in distributive conflict - compared to

\footnotetext{
${ }^{31}$ Formally, in any $t$, let $\omega_{t}^{\max }=\max _{\nu \in \mathcal{N}_{t}} \omega_{t}^{\nu}$ and let $\left\{\alpha_{0}, \ldots, \alpha_{n}\right\}$ be a set of real numbers such that $\alpha_{0}=0, \alpha_{n}=1$, and $\alpha_{i-1}<\alpha_{i}$ for all $1 \leq i \leq n$. Then let $I_{0}=0$ and $I_{i}=$ $\left(\alpha_{i-1} \omega_{t}^{\max }, \alpha_{i} \omega_{t}^{\max }\right]$ for all $i, 1 \leq i \leq n$. If $\omega_{t}^{\nu} \in I_{i}, i \geq 0$, then $N_{t}^{\nu} \equiv \#\left\{\nu^{\prime} \in \mathcal{N}_{t} \mid \nu^{\prime} \in I_{i}\right\}$.

${ }^{32}$ Formally, if $\nu \in C_{t}^{i}, i \in\{1,2,3,4\}, N_{t}^{\nu} \equiv \#\left\{\nu^{\prime} \in \mathcal{N}_{t} \mid \nu^{\prime} \in C_{t}^{i}\right\}$.
} 
class solidarity among propertyless agents - that exploitative relations persist over time as an equilibrium feature of capitalist economies.

Second, far from being metaphysical, the concept of exploitation provides the foundations for a logically coherent and empirically relevant analysis of inequalities and class relations in advanced capitalist economies. An index that identifies the exploitation level, or intensity of each individual can be defined and its empirical distribution studied using the standard tools of the theory of inequality measurement. In our analysis, we have focused on the Gini coefficient for illustrative purposes as it is one of the most widely used indices of dispersion, but - as our simulations show - the Gini coefficient does not necessarily convey clear normative information about the distribution of the exploitation index. An interesting open question concerns precisely the appropriate aggregate index of the intensity of exploitation. ${ }^{33}$

It would be interesting to extend our analysis to economies with $n$ goods, more general technologies, and even more complex bargaining procedures. A particularly intriguing question, for example, concerns the relation between power and technical change, and their joint relevance for class and exploitation. For the use of economic resources in distributive conflict is only one dimension of capitalist power. Control over the means of production implies that capitalists can also determine - at least to some extent - the direction and nature of technical change. In line with the recent literature on power-biased technical change (e.g., Guy and Skott [9, 10]), one could analyse innovations that allow capitalists - either directly or indirectly via changes in the structure of production - to alter power relations, for example, by affecting class solidarity and increasing the relative weight of economic resources, $\epsilon{ }^{34}$

Another important question concerns the implications of heterogeneity in labour skills and in individual consumption/leisure trade-offs. On the one hand, as already noted, the assumption that agents have the same skills and preferences, and perform the same amount of labour leads to less extreme inequalities in exploitation intensity than are observed in empirical income or wealth distributions. We interpret our results as identifying a lower bound to the intensity of exploitative relations, and as such we think that the distributions obtained in our models are quite remarkable. But it would be interesting to analyse the distributions emerging when this constraint is relaxed.

\footnotetext{
${ }^{33}$ The answer to this question depends on the normative insights that the notion exploitation as the unequal exchange of labour is meant to capture. We analyse this issue in $[22]$.

${ }^{34}$ Or, perhaps more subtly, using a divide-and-conquer strategy that changes the relevant $N_{t}^{\nu}$ for propertyless workers.
} 
On the other hand, as is well known, the introduction of heterogeneous labour poses major problems in the standard definition of labour embodied $v_{t}$. In this respect, it is important to stress that if the approach developed in Veneziani and Yoshihara [19, 21] is adopted, then the definition of the exploitation intensity index can be extended to economies with $n$ goods, general technologies, and heterogeneous labour, preferences and skills, and exploitative relations can be analysed empirically based on available data.

Finally, it would be interesting to consider more sophisticated norms in consumption behaviour, possibly introducing some (class-based) heterogeneity across agents. In our model, an increase in profits - and therefore exploitation - tends to raise the rate of accumulation. As already noted, however, Piketty [12] has shown that advanced capitalist economies are witnessing increases in profits (and the profit share) and in inequalities with a comparatively low rate of growth. The computational results in section 8 suggest that this may be the product of a structural change from a regime with a strong working class to one of capitalist dominance. Yet observed changes in consumption habits may also have played an important role. In particular, a norm of conspicuous consumption for the richest segments of society would imply that increases in profits are not necessarily translated into a higher rate of accumulation. Within our computational framework, this phenomenon could be captured by assuming individual, rather than average, consumption to depend on wealth: $b_{t}^{\nu}=f\left(\omega_{t-1}^{\nu}\right)$, with $f$ increasing and possibly convex.

Although it does not provide answers to these questions, this paper provides a conceptual and analytical framework to tackle them.

\section{References}

[1] Allegretto, S.A. 2011. The State of Working America's Wealth, 2011. EPI Briefing Paper. March 23, 2011.

[2] Cogliano, J.F., 2013. New Directions in Political Economy: Value Theory, Agent-Based Computational Modeling, and the Dynamics of Labor Mobility. Ph.D. Thesis. New School for Social Research.

[3] Cogliano, J.F., Jiang, X., 2014. Agent-Based Computational Economics: Simulation Tools for Heterodox Research. In F. Lee and B. Cronin (Eds.), Handbook of Research Methods and Applications in Heterodox Economics. Edward Elgar, Cheltenham. Forthcoming. 
[4] Devine, J., Dymski, G., 1991. Roemer's 'General' Theory of Exploitation is a Special Case. Economics and Philosophy 7, 235-275.

[5] Duménil, G., 1980. De la Valeur aux Prix de Production. Economica, Paris.

[6] Duménil, G., Foley, D.K., Lévy, D., 2009. A Note on the Formal Treatment of Exploitation in a Model with Heterogeneous Labor. Metroeconomica 60, 560-567.

[7] Flaschel, P., Franke, R., Veneziani, R., 2013. Labor Productivity and the Law of Decreasing Labor Content. Cambridge Journal of Economics 37, 379-402.

[8] Foley, D.K., 1982. The Value of Money, the Value of labour Power, and the Marxian Transformation Problem. Review of Radical Political Economics 14, 37-47.

[9] Guy, F., Skott, P., 2007. A Model of Power-Biased Technological Change. Economics Letters 95, 124-131.

[10] Guy, F., Skott, P., 2008. Power, productivity and profits. In M. Braham and F. Steffen (eds) Dimensions of Power: Concepts, Models, and Applications. Springer, Berlin.

[11] Mohun, S., 2004. The Labour Theory of Value as Foundation for Empirical Investigations. Metroeconomica 55, 65-95.

[12] Piketty, T., 2014. Capital in the Twenty-First Century. Harvard University Press, Cambridge, MA.

[13] Roemer, J.E., 1981. Analytical foundations of Marxian economic theory. Harvard University Press, Cambridge, MA.

[14] Roemer, J.E., 1982. A General Theory of Exploitation and Class. Harvard University Press, Cambridge, MA.

[15] Skillman, G.L., 1995. Ne Hic Saltaveris: the Marxian Theory of Exploitation after Roemer. Economics and Philosophy 11, 309-331.

[16] Takamasu, A., 2008. Classes in a virtual economy. Mimeo, Kansai University. 
[17] Veneziani, R., 2007. Exploitation and Time. Journal of Economic Theory 132, 189-207.

[18] Veneziani, R., 2013. Exploitation, Inequality, and Power. Journal of Theoretical Politics 25, 526-545.

[19] Veneziani, R., Yoshihara, N., 2014a. Exploitation in economies with heterogeneous preferences, skills and assets: An axiomatic approach. Journal of Theoretical Politics, forthcoming.

[20] Veneziani, R., Yoshihara, N., 2014b. Globalisation and Inequality: A Dynamic General Equilibrium Model of Unequal Exchange. Mimeo, QMUL and Hitotsubashi University.

[21] Veneziani, R., Yoshihara, N., 2014c. One million miles to go: taking the axiomatic road to defining exploitation. Mimeo, QMUL and Hitotsubashi University.

[22] Veneziani, R., Yoshihara, N., 2014d. The theory of exploitation as the unequal exchange of labour. Mimeo, QMUL and Hitotsubashi University.

[23] Wright, I. 2008. The Emergence of the Law of Value in a Dynamic Simple Commodity Economy. Review of Political Economy 20, 367-391.

[24] Wright, I. 2011a, Classical macrodynamics and the labor theory of value. Open Discussion Papers in Economics, no. 76. The Open University, Milton Keynes.

[25] Wright, I. 2011b, Convergence to natural prices in simple production. Open Discussion Papers in Economics, no. 75. The Open University, Milton Keynes.

[26] Yoshihara, N., 2010. Class and Exploitation in General Convex Cone Economies. Journal of Economic Behavior \& Organization 75, 281-296.

[27] Yoshihara, N., Veneziani, R., 2009. Exploitation as the Unequal Exchange of Labour: An Axiomatic Approach. WP N.655, Queen Mary University of London. 


\section{Appendix A}

\section{A.1 Equilibrium conditions}

The Figures in this section show that, in all of our simulations, conditions (b)-(d) of Definition 1 in the paper are satisfied, and so we are analysing the equilibrium dynamics of the economies considered.

Figure 18: Equilibrium conditions - Basic model

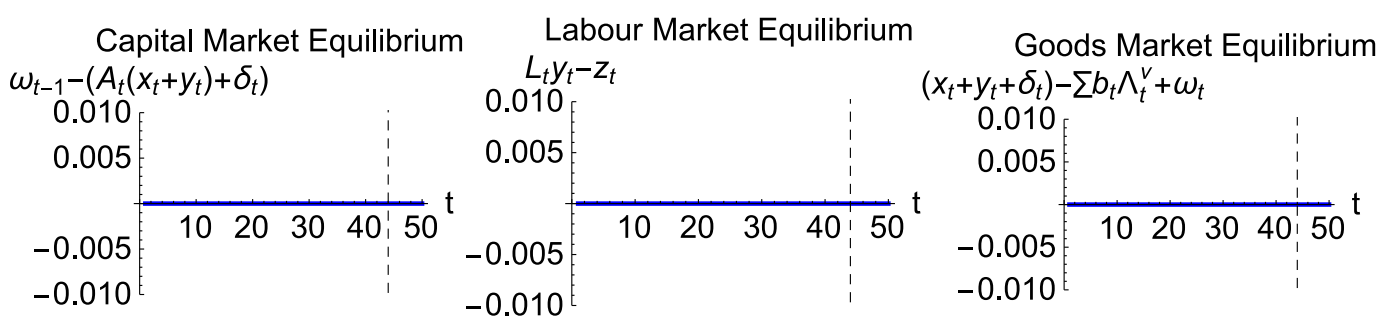

Figure 19: Equilibrium conditions - Model with endogenous subsistence and technical change

Capital Market Equilibrium
$\begin{gathered}\omega_{t-1}-\left(A_{t}\left(x_{t}+y_{t}\right)+\delta_{t}\right) \\ 0.010\end{gathered}$
0.005


Figure 20: Equilibrium conditions - Bargaining model with $\epsilon=0$

\begin{tabular}{|c|c|c|c|c|c|}
\hline Cap & tal Market Equilibrium & & our Market Equilibrium & & rium \\
\hline $\begin{array}{c}\omega_{t-1}-\left(A_{t}(x\right. \\
0.010\end{array}$ & $\left.\left.+y_{t}\right)+\delta_{t}\right)$ & $\begin{array}{r}L_{t} y_{t} \\
0.010\end{array}$ & & $\begin{array}{r}\left(x_{t}+y_{t}+\delta_{t}\right)- \\
0.010\end{array}$ & ${ }_{t} \wedge_{t}^{v}+\omega_{t}$ \\
\hline 0.005 & & 0.005 & & 0.005 & \\
\hline $\begin{array}{l}-0.005 \\
-0.010^{0}\end{array}$ & 102030405 & $\begin{array}{l}-0.005 \\
-0.010\end{array}$ & $\begin{array}{lllll}10 & 20 & 30 & 40 & 50\end{array}{ }^{\mathrm{t}}$ & -0.005 & $1020304050^{t}$ \\
\hline
\end{tabular}

Figure 21: Equilibrium conditions - Bargaining model with $\epsilon=1$

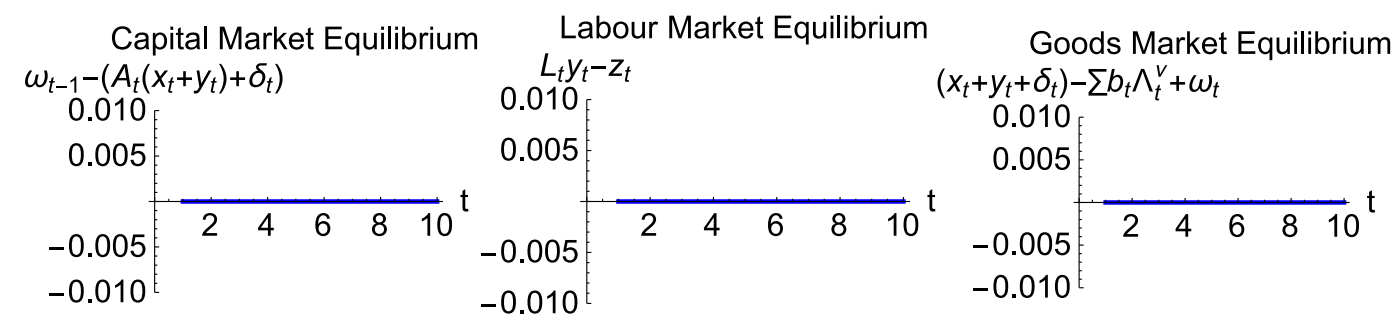

Figure 22: Equilibrium conditions - Bargaining model with $\epsilon=0.002$

Capital Market Equilibrium
$\omega_{t-1}-\left(A_{t}\left(x_{t}+y_{t}\right)+\delta_{t}\right)$
0.010
0.005




\section{A.2 An alternative analysis of technical change}

In this section, we analyse a variant of the model in section 7 of the paper in which technical change is modelled in a slightly different way.

We assume that, as soon as capitalists realise that $\pi^{\left(\widehat{w}_{t} ; A_{t-1}, L_{t-1}\right)}<\pi^{*}$, a new technique $\left(A^{\prime}, L^{\prime}\right)$ is chosen by first randomly selecting a change in $A_{t}$ from a uniform distribution between 0.03 and 0.05 . Then the new value of $L_{t}$ is determined by setting $v_{t} b_{t}=v_{1} b_{1}+h$, where $b_{t}$ is given by equation (6) in the paper and $h$ is a random real number in the interval $[-0.25,0.25]$. This ensures that the condition $1>v_{t} b_{t}$ is never violated and it helps new techniques "restore profitability" without imposing it by fiat. To ensure that $A_{t}<1$ for all $t$ there is a maximum value set at $A_{\max }=0.991$. Once $A_{\max }$ is reached, technical change continues as Harrod-neutral (constant $A_{\max }$ and decreasing $L_{t}$ when $\left.\pi^{\left(\widehat{w}_{t} ; A_{t-1}, L_{t-1}\right)}<\pi^{*}\right)$ to ensure that $1>v_{t} b_{t}$, but this phase is only run for a short time in order to focus on the Marx-biased phase of technical change.

All parameters, initial conditions and the sequencing of the simulation routine are identical to the model in section 7.1 of the paper, except for the length of time $T$, which is set to 150 .

The results of this model are strikingly similar to those reported in section 7.1 of the paper. Figure 23 shows the dynamics of the main variables of the model. The period in which $A_{t}=A_{\max }=0.991$ is denoted by a vertical dashed line. It is worth noting in passing that although $z_{t}$ and $\pi_{t}$ seem to vanish in the long-run, neither actually reaches zero: the minimum values of $z_{t}$ and $\pi_{t}$ are, respectively, 0.161723 and 0.00145133 . Observe further that Figure 23 displays growth cycles deriving from the interaction of capital accumulation and technical change. The fluctuations become increasingly violent and ultimately the economy settles on a course with very low net output and labour supply due to the increases in productivity. Figure 24 shows the evolution of $A_{t}, L_{t}$, and $v_{t}$. 
Figure 23: Summary results
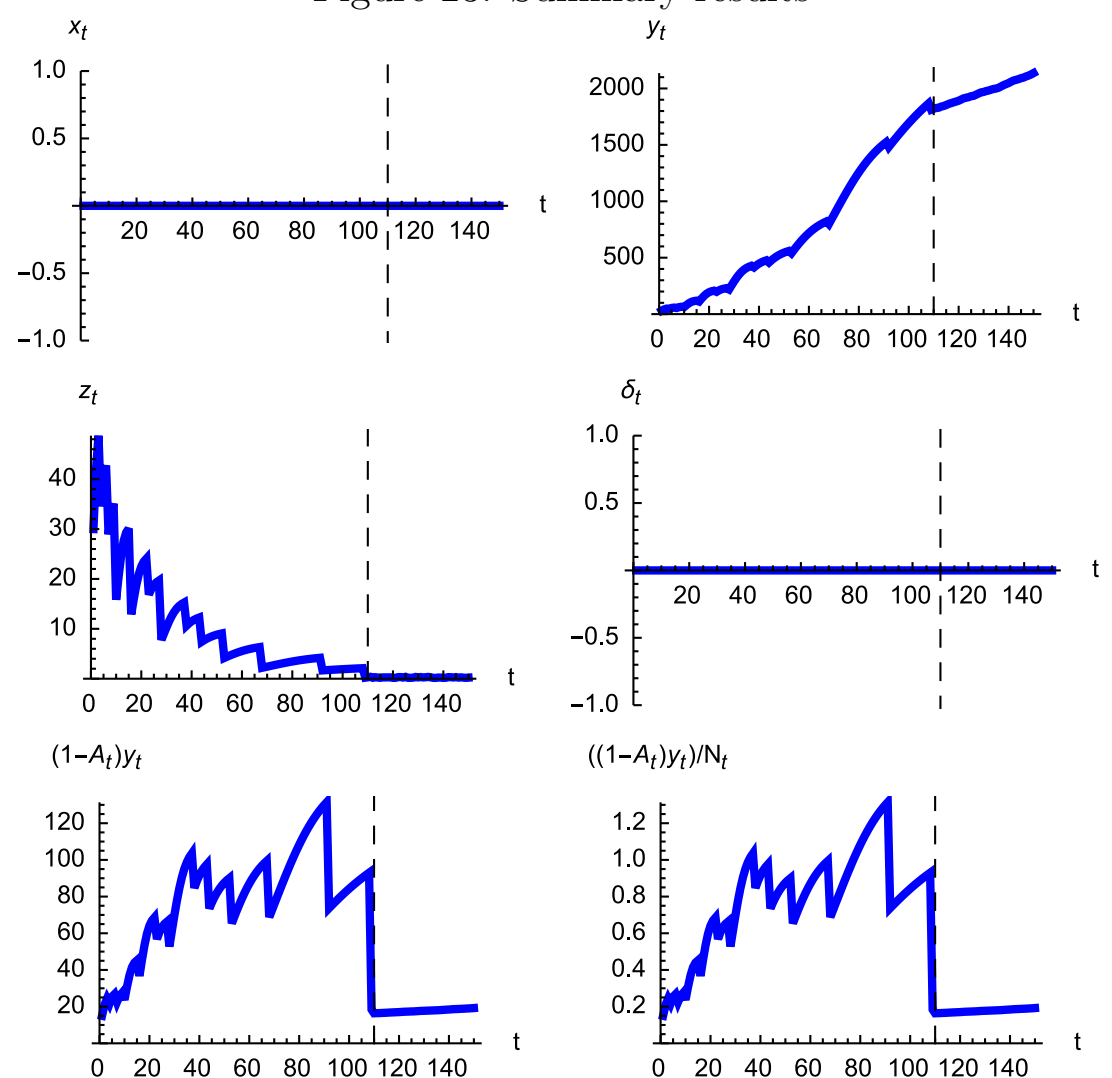

$\left(\left(1-A_{t}\right) y_{t}\right) / \mathrm{N}_{t}$

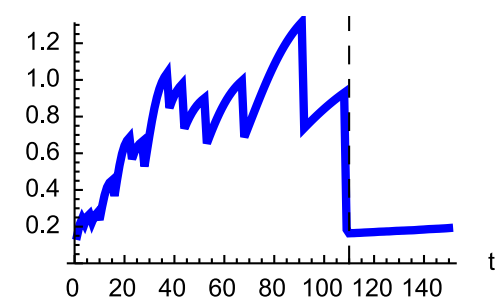

$W_{t-1}=p_{t-1} \omega_{t-1}$
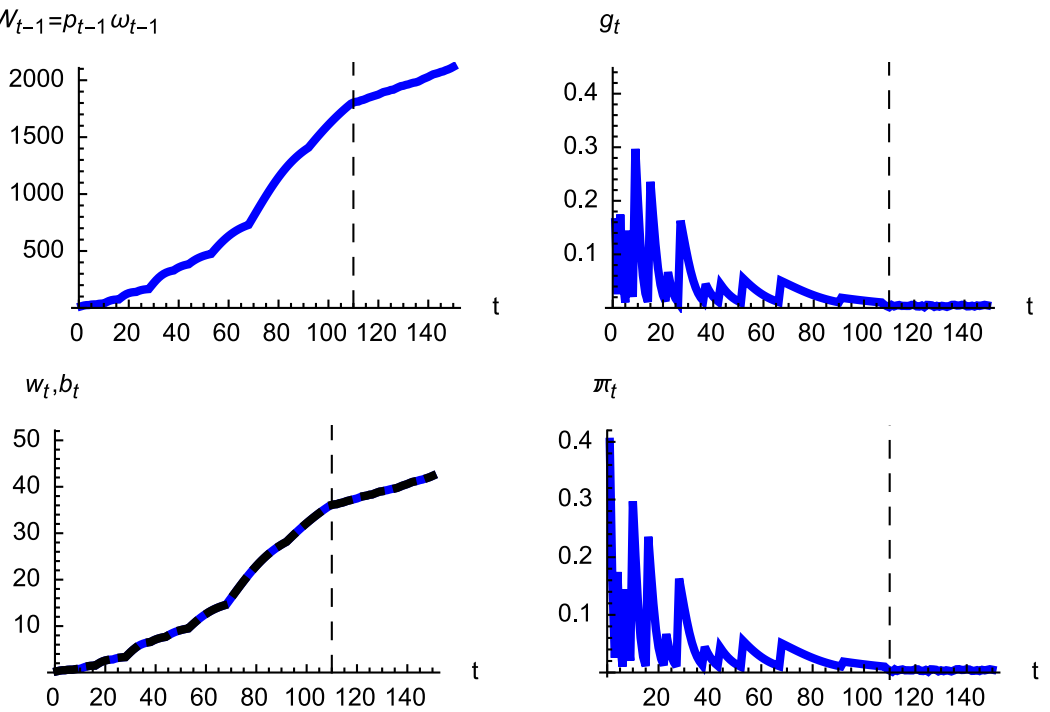
Figure 24: Technology and labour values
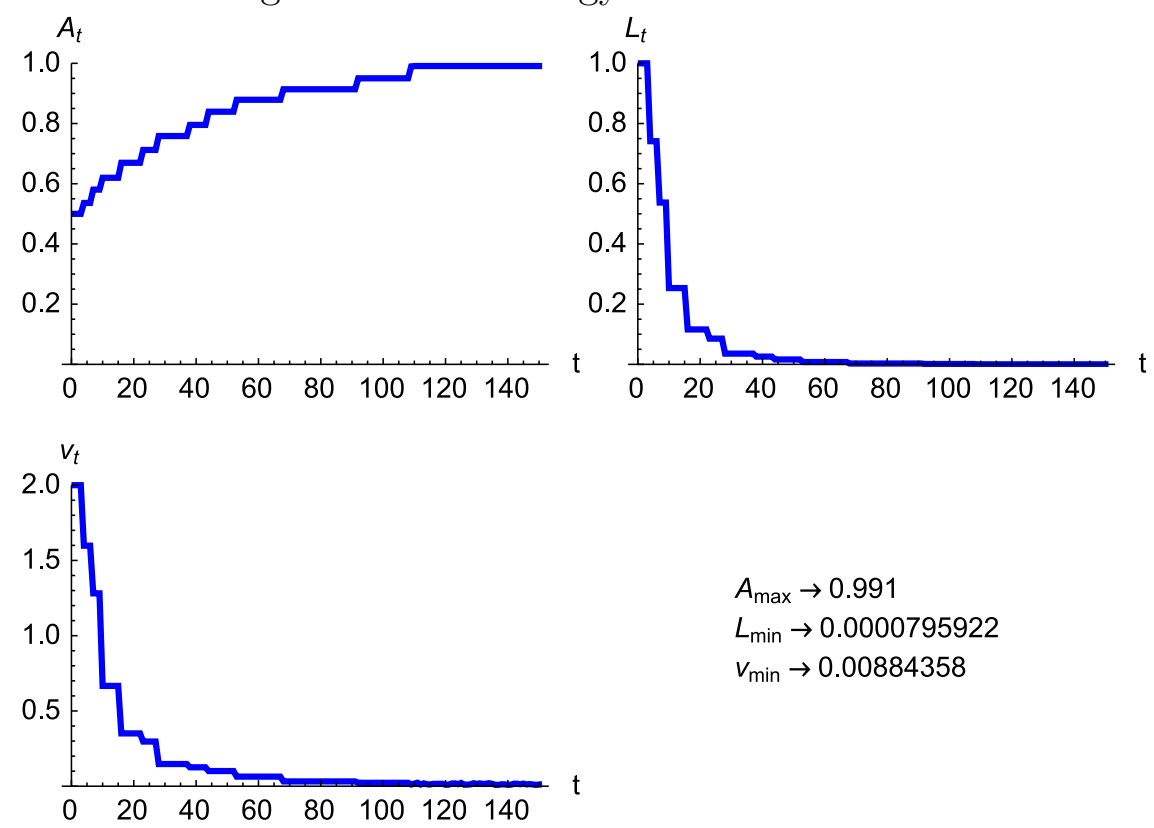

$$
\begin{aligned}
& A_{\max } \rightarrow 0.991 \\
& L_{\min } \rightarrow 0.0000795922 \\
& V_{\min } \rightarrow 0.00884358
\end{aligned}
$$

Figure 25(a) shows that the basic structure of exploitative relations is identical to that in section 7.1. The class structure of the economy, instead is slightly different: as shown in Figures 25(b) and 25(c), the number of agents in the top classes decreases over time and eventually goes to zero. This interesting result is the product of rapid technical change, which leads $L_{t}$ to decrease faster than capital accumulation, yielding a progressive decrease in labour demand. 
Figure 25: Class and exploitation status

(a) Exploitation status

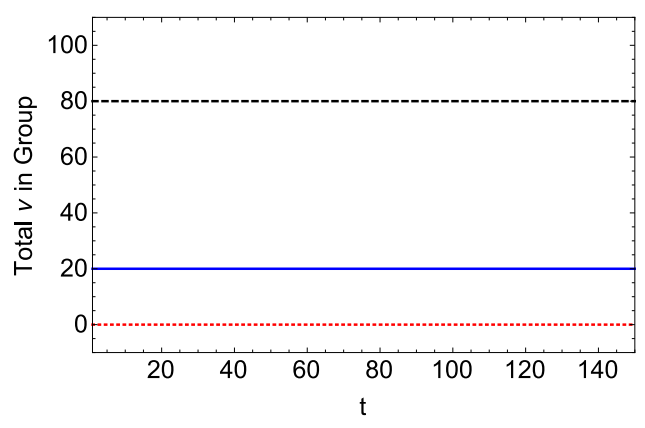

- Exploiters $\quad$-..-. Neither Exploiter or Exploited (b) Class status

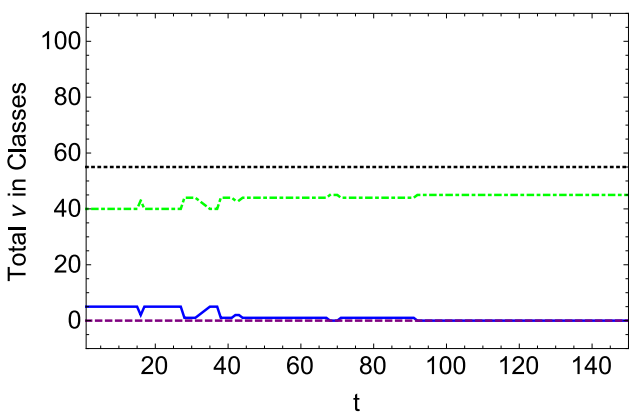

$\begin{aligned}-\mathrm{C}_{\mathrm{t}}^{1} & =\left\{(+,+, 0) \backslash(+, 0,0) ; \mathrm{L}_{\mathrm{t}} \mathrm{y}_{\mathrm{t}}^{v}>\mathrm{l}^{v}\right\} \\ \cdots \mathrm{C}_{\mathrm{t}}^{2} & =\left\{(+, 0,0) ; \mathrm{L}_{\mathrm{t}} \mathrm{y}_{\mathrm{t}}^{v} \leq 1^{v}\right\} \\ \cdots-. \mathrm{C}_{\mathrm{t}}^{3} & =\emptyset \\ \ldots . . \mathrm{C}_{\mathrm{t}}^{4} & =\left\{(0,0,+) ; \mathrm{W}_{\mathrm{t}-1}^{v}=0\right\}\end{aligned}$

(c) CECP

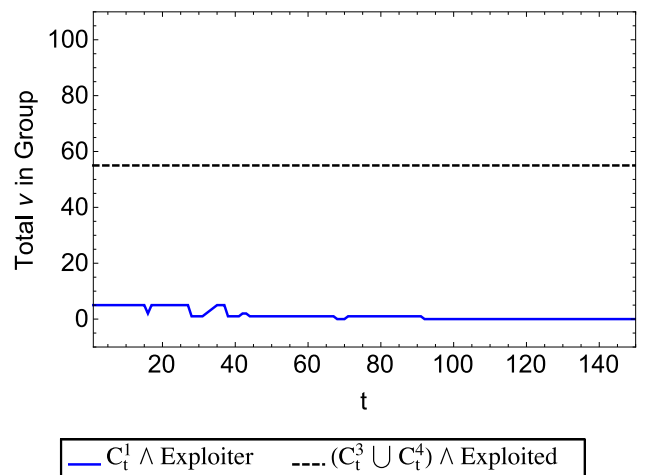

Figures 26(a) and 26(b) depict similar "exploitation cycles" as in section 7.1, which are induced by the interaction of accumulation and technical change. 
Figure 26: Exploitation intensity index

(a) Exploitation intensity index across agents

(b) Gini coefficient of $e_{t}^{\nu}$
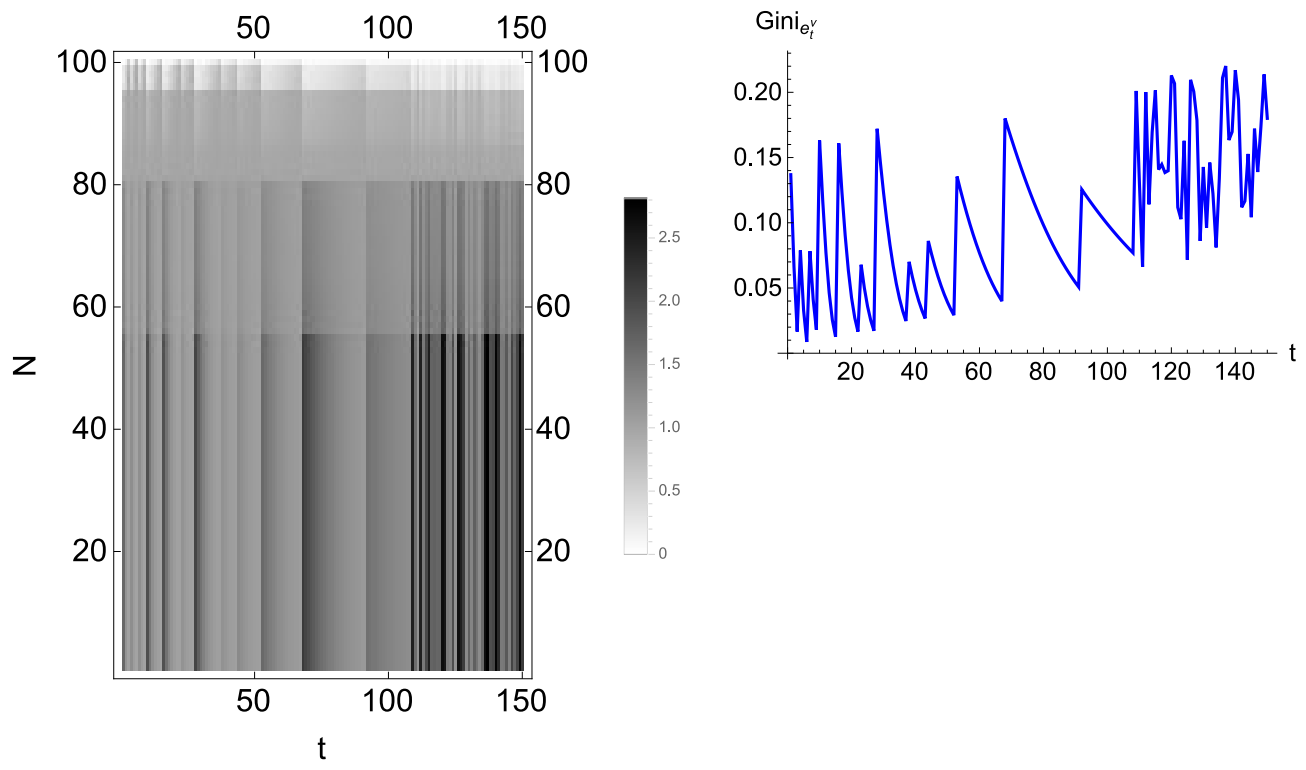

\section{A.3 An alternative representation of class}

In the paper, we follow Roemer [14] and define the main classes as follows:

$C_{t}^{1}=\left\{\nu \in \mathcal{N} \mid \mathcal{A}^{\nu}\left(p_{t}, w_{t}\right)\right.$ has a solution of the form $\left.(+,+, 0) \backslash(+, 0,0)\right\}$, $C_{t}^{2}=\left\{\nu \in \mathcal{N} \mid \mathcal{A}^{\nu}\left(p_{t}, w_{t}\right)\right.$ has a solution of the form $\left.(+, 0,0)\right\}$, $C_{t}^{3}=\left\{\nu \in \mathcal{N} \mid \mathcal{A}^{\nu}\left(p_{t}, w_{t}\right)\right.$ has a solution of the form $\left.(+, 0,+) \backslash(+, 0,0)\right\}$, $C_{t}^{4}=\left\{\nu \in \mathcal{N} \mid \mathcal{A}^{\nu}\left(p_{t}, w_{t}\right)\right.$ has a solution of the form $\left.(0,0,+)\right\}$.

In Corollary 1, we prove that at all equilibria with $\pi_{t}>0$, the class status of an agent is determined by the difference between labour demand and potential labour supply, which coincides with the agent's labour endowment.

Yet, one may argue that classes should be defined based on agents' actual choices, and in particular on their choices within labour relations. Therefore in this section we analyse the class structure and the relation between class and exploitation status in all of our models based on agent's actual choices. The results are remarkably similar to those obtained in the paper.

For the basic model: 
Figure 27: Class status (alternative definition) - Basic model

(a) Class status

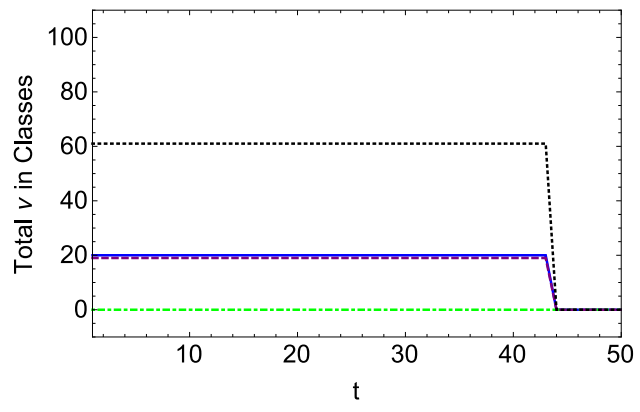

$-\mathrm{C}_{\mathrm{t}}^{1}=\left\{(+,+, 0) \backslash(+, 0,0) ; \mathrm{L}_{\mathrm{t}} \mathrm{y}_{\mathrm{t}}^{v}>\mathrm{z}_{\mathrm{t}}^{v}\right\}$

$\mathrm{C}_{\mathrm{t}}^{2}=\left\{(+, 0,0) ; \mathrm{L}_{\mathrm{t}} \mathrm{y}_{\mathrm{t}}^{v}=\mathrm{z}_{\mathrm{t}}^{v}\right\}$

$-\mathrm{C}_{\mathrm{t}}^{3}=\left\{(+, 0,+)(+, 0,0) ; \mathrm{L}_{\mathrm{t}} \mathrm{y}_{\mathrm{t}}^{\gamma}<\mathrm{z}_{\mathrm{t}}^{\prime \prime}\right\}$

$\ldots . . . \mathrm{C}_{\mathrm{t}}^{4}=\left\{(0,0,+) ; \mathrm{W}_{\mathrm{t}-1}^{v}=0\right\}$ (b) CECP

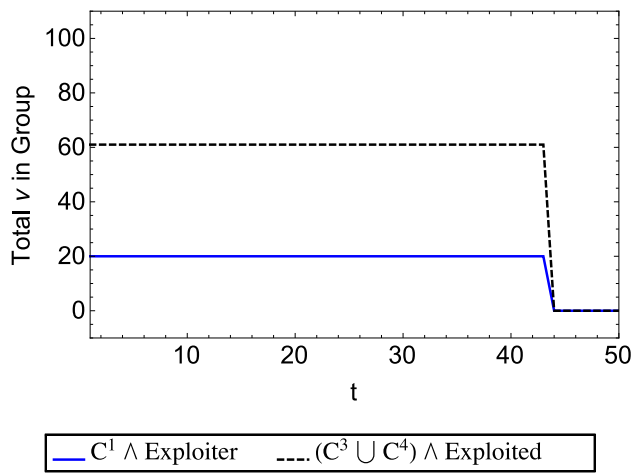

For the model with endogenous subsistence and technical change:

Figure 28: Class status (alternative definition) - Model with endogenous subsistence and technical change

(a) Class status

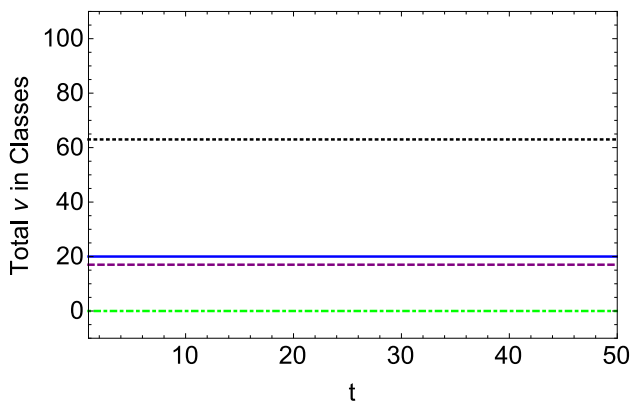

$\begin{aligned}-\mathrm{C}_{\mathrm{t}}^{1} & =\left\{(+,+, 0) \backslash(+, 0,0) ; \mathrm{L}_{\mathrm{t}} \mathrm{y}_{\mathrm{t}}^{v}>\mathrm{z}_{\mathrm{t}}^{v}\right\} \\ \mathrm{C}_{\mathrm{t}}^{2} & =\left\{(+, 0,0) ; \mathrm{L}_{\mathrm{t}} \mathrm{y}_{\mathrm{t}}^{v}=\mathrm{z}_{\mathrm{t}}^{v}\right\} \\ --\mathrm{C}_{\mathrm{t}}^{3} & =\left\{(+, 0,+) \backslash(+, 0,0) ; \mathrm{L}_{\mathrm{t}} \mathrm{y}_{\mathrm{t}}^{v}<\mathrm{z}_{\mathrm{t}}^{v}\right\} \\ \ldots \ldots \mathrm{C}_{\mathrm{t}}^{4} & =\left\{(0,0,+) ; \mathrm{W}_{\mathrm{t}-1}^{v}=0\right\}\end{aligned}$ (b) CECP

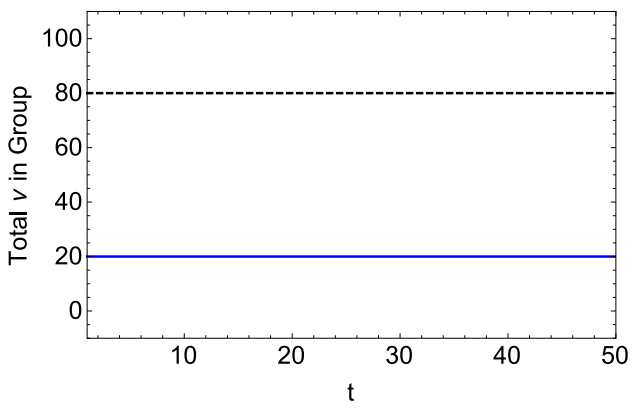

$-C_{t}^{1} \wedge$ Exploiter $\quad$-.-. $\left(C_{t}^{3} \cup C_{t}^{4}\right) \wedge$ Exploited

For the model with wage bargaining and $\varepsilon=0$ : 
Figure 29: Class status (alternative definition) - Bargaining model with $\epsilon=0$

(a) Class status

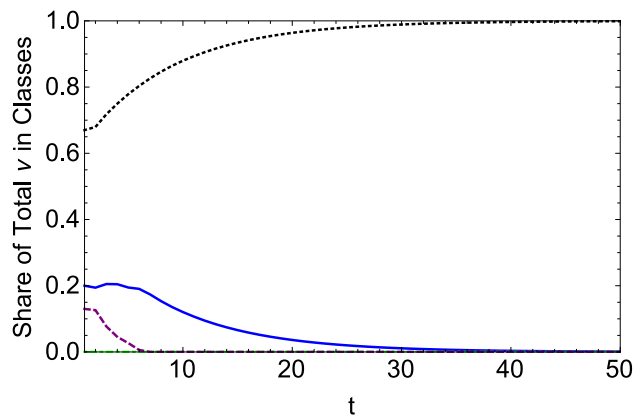

$-\mathrm{C}_{\mathrm{t}}^{1}=\left\{(+,+, 0) \backslash(+, 0,0) ; \mathrm{L}_{\mathrm{t}} \mathrm{y}_{\mathrm{t}}^{v}>\mathrm{z}_{\mathrm{t}}^{v}\right\}$

$\mathrm{C}_{\mathrm{t}}^{2}=\left\{(+, 0,0) ; \mathrm{L}_{\mathrm{t}} \mathrm{y}_{\mathrm{t}}^{v}=\mathrm{z}_{\mathrm{t}}^{v}\right\}$

$-C_{\mathrm{t}}^{3}=\left\{(+, 0,+)(+, 0,0) ; \mathrm{L}_{\mathrm{t}} \mathrm{y}_{\mathrm{t}}^{v}<\mathrm{z}_{\mathrm{t}}^{v}\right\}$

$\ldots . . . \mathrm{C}_{\mathrm{t}}^{4}=\left\{(0,0,+) ; \mathrm{W}_{\mathrm{t}-1}^{v}=0\right\}$ (b) CECP

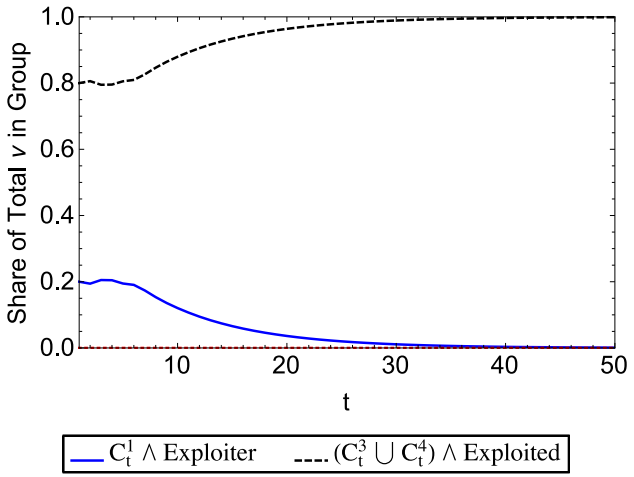

For the model with wage bargaining and $\varepsilon=1$ :

Figure 30: Class status (alternative definition) - Bargaining model with $\epsilon=1$

(a) Class status

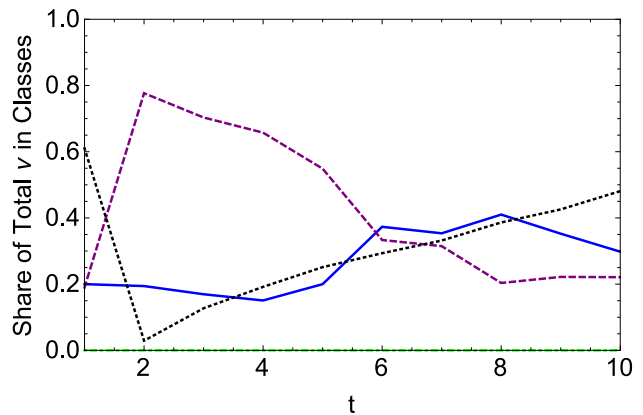

$$
\begin{aligned}
-\mathrm{C}_{\mathrm{t}}^{1} & =\left\{(+,+, 0) \backslash(+, 0,0) ; \mathrm{L}_{\mathrm{t}} \mathrm{t}_{\mathrm{t}}^{v}>\mathrm{z}_{\mathrm{t}}^{v}\right\} \\
\mathrm{C}_{\mathrm{t}}^{2} & =\left\{(+, 0,0) ; \mathrm{L}_{\mathrm{t}} \mathrm{y}_{\mathrm{t}}^{v}=\mathrm{z}_{\mathrm{t}}^{v}\right\} \\
-\cdots-\mathrm{C}_{\mathrm{t}}^{3} & =\left\{(+, 0,+)(+, 0,0) ; \mathrm{L}_{\mathrm{t}} \mathrm{y}_{\mathrm{t}}^{v}<\mathrm{z}_{\mathrm{t}}^{v}\right\} \\
\ldots-. . \mathrm{C}_{\mathrm{t}}^{4} & =\left\{(0,0,+) ; \mathrm{W}_{\mathrm{t}-1}^{v}=0\right\}
\end{aligned}
$$

(b) CECP

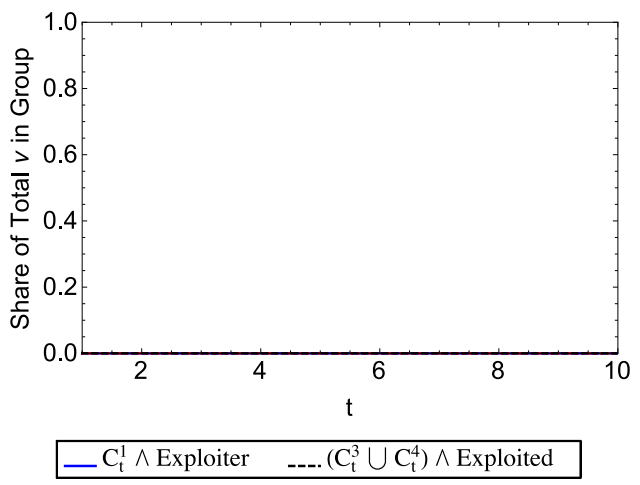

For the model with wage bargaining and $\varepsilon=0.002$ : 
Figure 31: Class status (alternative definition) - Bargaining model with $\epsilon=$ 0.002

(a) Class status

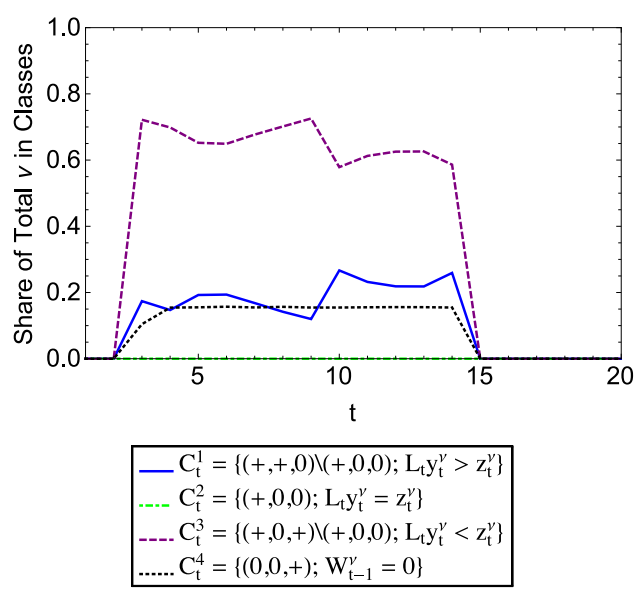

(b) CECP

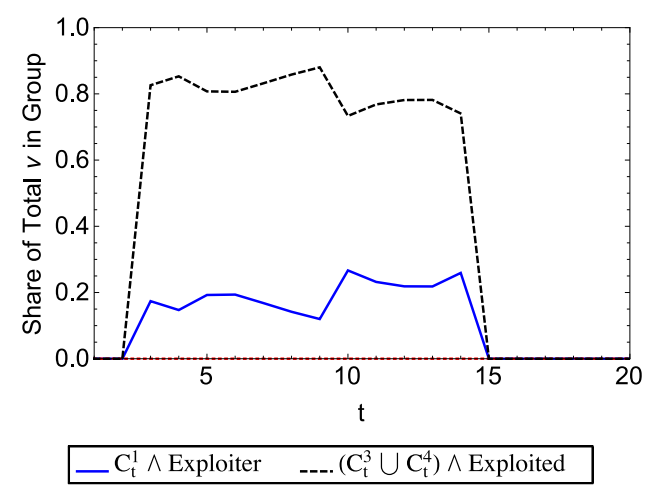

\section{A.4 Gini coefficients of wealth and income}

In section 8 of the paper, we refer to the behaviour of Gini coefficients for wealth and income (defined as the gross revenue of each agent, corresponding to the left hand side of constraint (1) of $M P_{t}^{\nu}$ ) over the course the simulations. The Figures below depict the evolution of these Gini coefficients.

For the model with wage bargaining and $\epsilon=0$ :

Figure 32: Gini coefficients of wealth and income - Bargaining model with $\epsilon=0$

(a) Gini coefficient of $\omega_{t-1}$

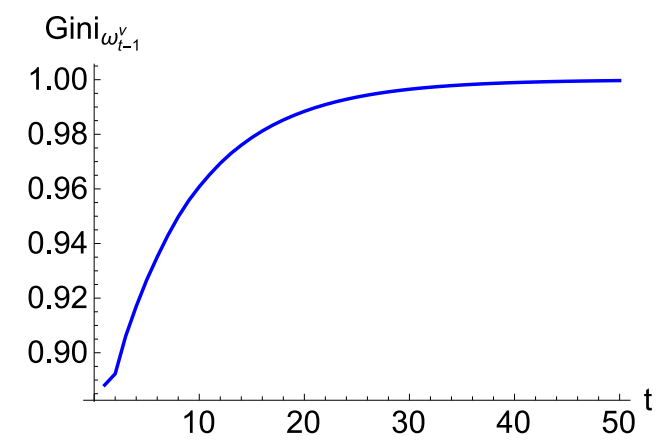

(b) Gini coefficient of income

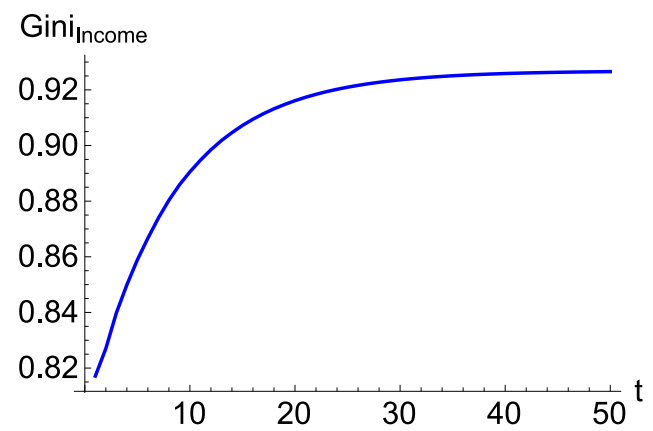

For the model with wage bargaining and $\epsilon=1$ : 
Figure 33: Gini coefficients of wealth and income - Bargaining model with $\epsilon=1$

(a) Gini coefficient of $\omega_{t-1}$

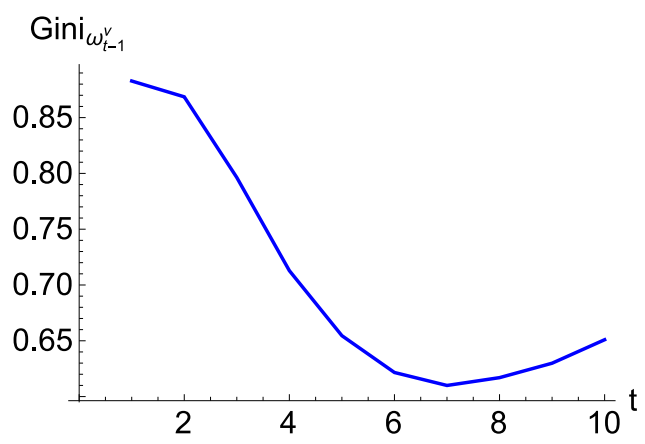

(b) Gini coefficient of income

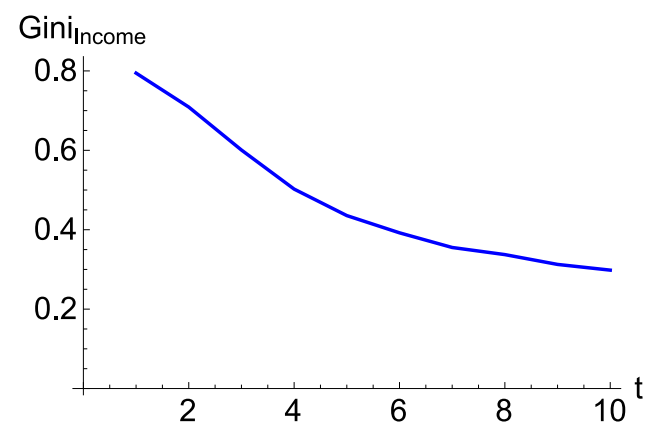

For the model with wage bargaining and $\epsilon=0.002$ :

Figure 34: Gini coefficients of wealth and income - Bargaining model with $\epsilon=0.002$

(a) Gini coefficient of $\omega_{t-1}$

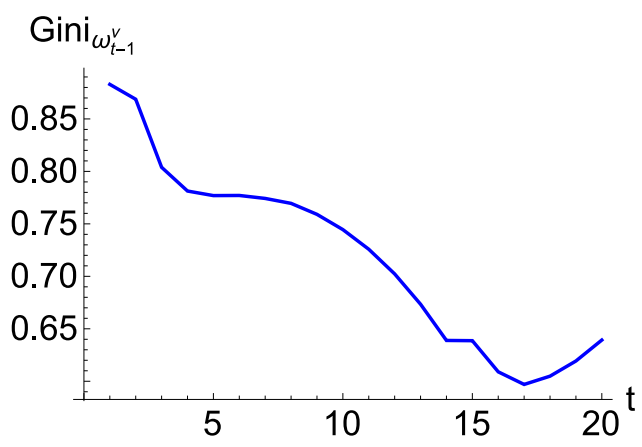

(b) Gini coefficient of income

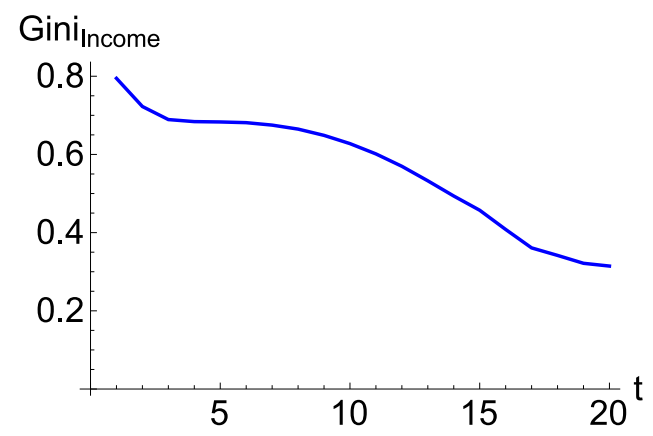

